ESAIM: M2AN 47 (2013) 1077-1106

DOI: $10.1051 / \mathrm{m} 2 \mathrm{an} / 2012058$
ESAIM: Mathematical Modelling and Numerical Analysis

www.esaim-m2an.org

\title{
OPTIMAL CONTROL OF THE BIDOMAIN SYSTEM (III): EXISTENCE OF MINIMIZERS AND FIRST-ORDER OPTIMALITY CONDITIONS
}

\author{
Karl Kunisch ${ }^{1}$ AND MarCus WAGNER ${ }^{2}$
}

\begin{abstract}
We consider optimal control problems for the bidomain equations of cardiac electrophysiology together with two-variable ionic models, e.g. the Rogers-McCulloch model. After ensuring the existence of global minimizers, we provide a rigorous proof for the system of first-order necessary optimality conditions. The proof is based on a stability estimate for the primal equations and an existence theorem for weak solutions of the adjoint system.
\end{abstract}

Mathematics Subject Classification. 35G31, 35Q92, 49J20, 49K20, 92C30.

Received December 27, 2011.

Published online June 13, 2013.

\section{INTRODUCTION}

In this work, we continue our investigations of optimal control problems for the bidomain system. After the study of the monodomain approximation of the equations and a thorough stability and regularity analysis of weak solutions for the full bidomain equations, as contained in the previous papers $[11,12]$, we are now in position to analyze the related control problems with respect to the existence of minimizers as well as to provide a rigorous proof of the first-order necessary optimality conditions.

Let $\Omega \subset \mathbb{R}^{3}$ be a bounded domain and $T>0$ a fixed time horizon. Then the bidomain system, representing a well-accepted description of the electrical activity of the heart, is given by ${ }^{3}$

$$
\begin{aligned}
& \frac{\partial \Phi_{\mathrm{tr}}}{\partial t}+I_{\mathrm{ion}}\left(\Phi_{\mathrm{tr}}, W\right)-\operatorname{div}\left(M_{i} \nabla \Phi_{i}\right)=\quad I_{i} \text { for almost all }(x, t) \in \Omega \times[0, T] ; \\
& \frac{\partial \Phi_{\mathrm{tr}}}{\partial t}+I_{\mathrm{ion}}\left(\Phi_{\mathrm{tr}}, W\right)+\operatorname{div}\left(M_{e} \nabla \Phi_{e}\right)=-I_{e} \text { for almost all }(x, t) \in \Omega \times[0, T] ; \\
& \frac{\partial W}{\partial t}+G\left(\Phi_{\mathrm{tr}}, W\right) \\
& =\quad 0 \text { for almost all }(x, t) \in \Omega \times[0, T] ;
\end{aligned}
$$

Keywords and phrases. PDE constrained optimization, bidomain equations, two-variable ionic models, weak local minimizer, existence theorem, necessary optimality conditions, pointwise minimum condition.

1 University of Graz, Institute for Mathematics and Scientific Computing, Heinrichstraße 36, 8010 Graz, Austria. karl.kunisch @ uni-graz.at

2 University of Leipzig, Department of Mathematics, P. O. B. 1009 20, 04009 Leipzig, Germany.

www . thecitytocome.de/marcus . wagner@math . uni-leipzig.de

${ }^{3}$ The bidomain model has been considered first in [21]. A detailed introduction may be found e.g. in [20], pp. 21-56. 


$$
\begin{aligned}
& \mathfrak{n}^{\mathrm{T}} M_{i} \nabla \Phi_{i}=0 \quad \text { for all }(x, t) \in \partial \Omega \times[0, T] ; \\
& \mathfrak{n}^{\mathrm{T}} M_{e} \nabla \Phi_{e}=0 \text { for all }(x, t) \in \partial \Omega \times[0, T] ; \\
& \Phi_{\operatorname{tr}}(x, 0)=\Phi_{i}(x, 0)-\Phi_{e}(x, 0)=\Phi_{0}(x) \text { and } W(x, 0)=W_{0}(x) \text { for almost all } x \in \Omega .
\end{aligned}
$$

In this model, $\Omega$ represents the spatial domain occupied by the cardiac muscle, the variables $\Phi_{i}$ and $\Phi_{e}$ denote the intracellular and extracellular electric potentials, and $\Phi_{\mathrm{tr}}=\Phi_{i}-\Phi_{e}$ is the transmembrane potential. The anisotropic electric properties of the intracellular and the extracellular tissue parts are modeled by conductivity tensors $M_{i}$ and $M_{e}$. The specification of the model for the ionic current $I_{\text {ion }}$ in (1.1) and (1.2) and the gating function $G$ in (1.3) will be made below. We shall consider three so-called two-variable models wherein $I_{\text {ion }}$ and $G$ depend on $\Phi_{\mathrm{tr}}$ as well as on a single gating variable $W$, which describes in a cumulative way the effects of the ion transport through the cell membranes (see Sect. 2.2.). Finally, the inhomogeneities $I_{i}$ and $I_{e}$ represent the intracellular and extracellular stimulation currents, respectively.

We shall investigate optimal control problems of the form

(P) $F\left(\Phi_{\mathrm{tr}}, \Phi_{e}, W, I_{e}\right)=\int_{0}^{T} \int_{\Omega} r\left(x, t, \Phi_{\operatorname{tr}}(x, t), \Phi_{e}(x, t), W(x, t)\right) \mathrm{d} x \mathrm{~d} t+\frac{\mu}{2} \int_{0}^{T} \int_{\Omega_{\mathrm{con}}} I_{e}(x, t)^{2} \mathrm{~d} x \mathrm{~d} t \longrightarrow \inf$ !

subject to the bidomain equations (1.1)-(1.6) in its weak formulation (see (2.1)-(2.4) below)

and the control restriction $I_{e} \in \mathcal{C}$

where $\Omega_{\text {con }}$ is a Lipschitz subdomain of $\Omega$ and

$$
\begin{aligned}
& \mathcal{C}=\left\{Q I \mid I \in L^{\infty}\left[(0, T), L^{2}(\Omega)\right],\right. \operatorname{supp}(I) \subseteq \Omega_{\text {con }} \times[0, T], \\
&\left.\left.|I(x, t)| \leqslant R(\forall)(x, t) \in \Omega_{T}\right\} \subset L^{\infty}\left[(0, T), L^{2}(\Omega)\right] .9\right)
\end{aligned}
$$

For the description of the control domain, the linear operator $Q: L^{2}\left(\Omega_{T}\right) \rightarrow L^{2}\left(\Omega_{T}\right)$ defined by

$$
Q I(x, t)=I(x, t)-\mathbb{1}_{\Omega_{\mathrm{con}}}(x) \cdot \frac{1}{\left|\Omega_{\mathrm{con}}\right|} \int_{\Omega_{\mathrm{con}}} I(\tilde{x}, t) \mathrm{d} \tilde{x}
$$

has been used. When applied to a function $I$ with $\operatorname{supp}(I) \subseteq \Omega_{\text {con }} \times[0, T], Q$ extends by zero the orthogonal projection onto the complement of the subspace $\left\{Z \mid \int_{\Omega_{\text {con }}} Z(\tilde{x}, t) \mathrm{d} \tilde{x}=0\right.$ for a.a. $\left.t \in(0, T)\right\} \subset$ $L^{2}\left[(0, T), L^{2}\left(\Omega_{\mathrm{con}}\right)\right]$. Consequently, for $I_{e} \in \mathcal{C}$, we have

$$
\int_{\Omega} I_{e}(x, t) \mathrm{d} x=\int_{\Omega_{\mathrm{con}}} I_{e}(x, t) \mathrm{d} x=0 \quad \text { for almost all } t \in(0, T),
$$

what guarantees the solvability of the state equations ( $c f$. Thm. 2.3 below). In problem $(\mathrm{P})$, the extracellular excitation $I_{e}$ acts as control, which is allowed to be applied on the subdomain $\Omega_{\text {con }}$ only. ${ }^{4}$ The pointwise constraint within the description (1.9) of $\mathcal{C}$ is included due to the obvious fact that one cannot apply arbitrary large electrical stimulations to living tissue without damaging it. In mathematical terms, this restriction is necessary in order to establish a stability estimate for the bidomain system (Thm. 2.4).

Due to the complex dynamical behaviour of the state equations, an appropriate choice of the integrand $r$ within the first term of the objective (1.7) for concrete applications is quite delicate. With arrhythmia or tachycardia in mind, it could be chosen as $r(x, t, \varphi, \eta, w)=\left(\varphi-\Phi_{\text {des }}(t)\right)^{2}$ where $\Phi_{\text {des }}$ denotes some desired

\footnotetext{
${ }^{4}$ For physiological reasons, the intracellular excitation $I_{i}$ must be set zero.
} 
trajectory for the controlled state $\Phi_{\mathrm{tr}}$, which is part of a solution of (1.1)-(1.5) as well, cf. [16]. The second term expresses the requirement that - regardless of whether the pointwise restriction within (1.9) is active - the overall stimulus should be as small as possible. Consequently, solutions with little intervention to the cardiac system are favored.

Besides an existence theorem for global minimizers (Thm. 3.4), the main result of the present paper is the rigorous proof of the following set of first-order necessary optimality conditions for sufficiently regular local minimizers $\left(\hat{\Phi}_{\mathrm{tr}}, \hat{\Phi}_{e}, \hat{W}, \hat{I}_{e}\right)$ of $(\mathrm{P})$, consisting of the variational inequality

$$
\int_{0}^{T} \int_{\Omega_{\mathrm{con}}}\left(\mu \hat{I}_{e}-Q P_{2}\right) \cdot\left(I_{e}-\hat{I}_{e}\right) \mathrm{d} x \mathrm{~d} t \geqslant 0 \quad \text { for all admissible controls } I_{e}
$$

and the adjoint system $^{5}$

$$
\begin{gathered}
\int_{0}^{T} \int_{\Omega}\left(-\frac{\partial P_{1}}{\partial t}+\frac{\partial I_{\mathrm{ion}}}{\partial \varphi}\left(\hat{\Phi}_{\mathrm{tr}}, \hat{W}\right) P_{1}+\frac{\partial G}{\partial \varphi}\left(\hat{\Phi}_{\mathrm{tr}}, \hat{W}\right) P_{3}\right) \psi \mathrm{d} x \mathrm{~d} t+\int_{0}^{T} \int_{\Omega} \nabla \psi^{\mathrm{T}} M_{i}\left(\nabla P_{1}+\nabla P_{2}\right) \mathrm{d} x \mathrm{~d} t(1.13) \\
=-\int_{0}^{T} \int_{\Omega}\left(\frac{\partial r}{\partial \varphi}\left(\hat{\Phi}_{\mathrm{tr}}, \hat{\Phi}_{e}, \hat{W}\right)\right) \psi \mathrm{d} x \mathrm{~d} t \quad \forall \psi \in L^{2}\left[(0, T), W^{1,2}(\Omega)\right], P_{1}(x, T) \equiv 0 ; \\
\int_{0}^{T} \int_{\Omega} \nabla \psi^{\mathrm{T}} M_{i} \nabla P_{1} \mathrm{~d} x \mathrm{~d} t+\int_{0}^{T} \int_{\Omega} \nabla \psi^{\mathrm{T}}\left(M_{i}+M_{e}\right) \nabla P_{2} \mathrm{~d} x \mathrm{~d} t=-\int_{0}^{T} \int_{\Omega} \frac{\partial r}{\partial \eta}\left(\hat{\Phi}_{\mathrm{tr}}, \hat{\Phi}_{e}, \hat{W}\right) \psi \mathrm{d} x \mathrm{~d} t \\
\quad \forall \psi \in L^{2}\left[(0, T), W^{1,2}(\Omega)\right] \text { with } \int_{\Omega} \psi(x, t) \mathrm{d} x=0 \text { for a.a. } t \in(0, T), \int_{\Omega} P_{2}(x, t) \mathrm{d} x=0(\forall) t \in(0, T) ; \\
\int_{0}^{T} \int_{\Omega}\left(-\frac{\partial P_{3}}{\partial t}+\frac{\partial I_{\mathrm{ion}}}{\partial w}\left(\hat{\Phi}_{\mathrm{tr}}, \hat{W}\right) P_{1}+\frac{\partial G}{\partial w}\left(\hat{\Phi}_{\mathrm{tr}}, \hat{W}\right) P_{3}\right) \psi \mathrm{d} x \mathrm{~d} t=-\int_{0}^{T} \int_{\Omega}\left(\frac{\partial r}{\partial w}\left(\hat{\Phi}_{\mathrm{tr}}, \hat{\Phi}_{e}, \hat{W}\right)\right) \psi \mathrm{d} x \mathrm{~d} t(1.15) \\
\forall \psi \in L^{2}\left[(0, T), L^{2}(\Omega)\right], P_{3}(x, T) \equiv 0
\end{gathered}
$$

for the multipliers $P_{1}, P_{2}$ and $P_{3}$ related to the weak state equations (2.1), (2.2) and (2.3) below, respectively (Thm. 5.2). The proof, which will be given by fitting the problem $(\mathrm{P})$ into the framework of weakly singular problems in the sense of Ito/Kunisch (see [9], p. $17 \mathrm{f}$.), is based on two main ingredients. The first one is a stability estimate for the primal equations (Thm. 2.4), whose proof has been already provided in the previous publication [12]. Secondly, we need an existence proof for weak solutions of the adjoint system, which is contained in the present paper (Thm. 4.2). In difference to the monodomain approximation considered in [11], the proof of the optimality conditions requires additional regularity of the minimizer $\left(\hat{\Phi}_{\mathrm{tr}}, \hat{\Phi}_{e}, \hat{W}, \hat{I}_{e}\right)$ in the case of the full bidomain system.

In the literature, only a few studies related to the optimal control of the bidomain system are available as yet, mostly restricted to the monodomain approximation. We mention $[1,5,10,13-16]$ and refer to [11], page 1527 , for a closer discussion. Numerical work concerning open-loop control of the bidomain equations with the goal of dampening of excitation and reentry waves has been realized in $[10,14-16]$. The problems were treated with gradient and Newton-type techniques applied to FEM discretizations of the state equations.

The paper is structured in the following way. In Section 2, the solution concepts for the bidomain equations are outlined. We present the ionic models to be used and summarize the existence and stability theorems for weak solutions of (1.1)-(1.6). Then, in Section 3, we restate the optimal control problem (1.7)-(1.8) within function spaces, subsequently analyzing the structure of the feasible domain and establishing the existence of global minimizers. Section 4 is concerned with the derivation of the adjoint system and the existence proof for a weak solution of it. Finally, in Section 5, we state and prove the first-order necessary optimality conditions for the control problem.

\footnotetext{
${ }^{5}$ Within the functions $r(x, t, \varphi, \eta, w), I_{\operatorname{ion}}(\varphi, w)$ and $G(\varphi, w)$, the real variables $\varphi, \eta$ and $w$ are the placeholders for $\Phi_{\operatorname{tr}}, \Phi_{e}$ and $W$, respectively.
} 


\section{Notations.}

We denote by $L^{p}(\Omega)$ the space of functions, which are in the $p$ th power integrable $(1 \leqslant p<\infty)$, or are measurable and essentially bounded $(p=\infty)$, and by $W^{1, p}(\Omega)$ the Sobolev space of functions $\psi: \Omega \rightarrow \mathbb{R}$ which, together with their first-order weak partial derivatives, belong to the space $L^{p}(\Omega, \mathbb{R})(1 \leqslant p<\infty)$. For spaces of Bochner integrable mappings, e.g. $L^{2}\left[(0, T), W^{1,2}(\Omega)\right]$, we refer to the summary in [11], page 1542 . $\Omega_{T}$ is an abbreviation for $\Omega \times[0, T]$. The gradient $\nabla$ is always taken only with respect to the spatial variables $x$. The characteristic function of the set $\mathrm{A} \subseteq \mathbb{R}^{3}$ is defined as $\mathbb{1}_{\mathrm{A}}: \mathbb{R}^{3} \rightarrow \mathbb{R}$ with $\mathbb{1}_{\mathrm{A}}(x)=1 \Longleftrightarrow x \in \mathrm{A}$ and $\mathbb{1}_{\mathrm{A}}(x)=0 \Longleftrightarrow x \notin \mathrm{A}$. Finally, the nonstandard abbreviation " $(\forall) t \in \mathrm{A}$ "has to be read as "for almost all $t \in \mathrm{A}$ " or "for all $t \in \mathrm{A}$ except for a Lebesgue null set", and the symbol $\mathfrak{o}$ denotes, depending on the context, the zero element or the zero function of the underlying space.

\section{WEAK SOLUTIONS OF THE BIDOMAIN SYSTEM}

\subsection{Parabolic-elliptic form of the bidomain system; strong and weak solutions}

It is well-known that the bidomain system (1.1)-(1.6) can be equivalently stated in parabolic-elliptic form, cf. [4], page 459, and [12], page 4, (2.1)-(2.9). In its weak formulation, the system reads as follows:

$$
\begin{gathered}
\int_{\Omega}\left(\frac{\partial \Phi_{\mathrm{tr}}}{\partial t} \cdot \psi+\nabla \psi^{\mathrm{T}} M_{i}\left(\nabla \Phi_{\mathrm{tr}}+\nabla \Phi_{e}\right)+I_{\mathrm{ion}}\left(\Phi_{\mathrm{tr}}, W\right) \psi\right) \mathrm{d} x=\int_{\Omega} I_{i} \psi \mathrm{d} x \\
\forall \psi \in W^{1,2}(\Omega), \text { for a.a. } t \in(0, T) ; \\
\int_{\Omega}\left(\nabla \psi^{\mathrm{T}} M_{i} \nabla \Phi_{\mathrm{tr}}+\nabla \psi^{\mathrm{T}}\left(M_{i}+M_{e}\right) \nabla \Phi_{e}\right) \mathrm{d} x=\int_{\Omega}\left(I_{i}+I_{e}\right) \psi \mathrm{d} x \\
\forall \psi \in W^{1,2}(\Omega) \text { with } \int_{\Omega} \psi(x) \mathrm{d} x=0, \text { for a.a. } t \in(0, T) ; \\
\int_{\Omega}\left(\frac{\partial W}{\partial t}+G\left(\Phi_{\mathrm{tr}}, W\right)\right) \psi \mathrm{d} x=0 \quad \forall \psi \in L^{2}(\Omega), \text { for a.a. } t \in(0, T) ; \\
\Phi_{\mathrm{tr}}(x, 0)=\Phi_{0}(x) \text { and } W(x, 0)=W_{0}(x) \quad \text { for almost all } x \in \Omega .
\end{gathered}
$$

Throughout the paper, the following assumptions about the data will be made:

Assumptions 2.1 (Basic assumptions on the data).

1) $\Omega \subset \mathbb{R}^{3}$ is a bounded Lipschitz domain.

2) $M_{i}, M_{e}: \operatorname{cl}(\Omega) \rightarrow \mathbb{R}^{3 \times 3}$ are symmetric, positive definite matrix functions with $L^{\infty}(\Omega)$-coefficients, obeying uniform ellipticity conditions:

$0 \leqslant \mu_{1}\|\xi\|^{2} \leqslant \xi^{\mathrm{T}} M_{i}(x) \xi \leqslant \mu_{2}\|\xi\|^{2}$ and $0 \leqslant \mu_{1}\|\xi\|^{2} \leqslant \xi^{\mathrm{T}} M_{e}(x) \xi \leqslant \mu_{2}\|\xi\|^{2} \quad \forall \xi \in \mathbb{R}^{3} \forall x \in \Omega$

with $\mu_{1}, \mu_{2}>0$.

The notions of strong and weak solutions are as follows:

\section{Definition 2.2.}

1) (Strong solution of the bidomain system $)^{6} \mathrm{~A}$ triple $\left(\Phi_{\mathrm{tr}}, \Phi_{e}, W\right)$ is called a strong solution of the bidomain system (2.1)-(2.4) on $[0, T]$ iff the functions $\Phi_{\mathrm{tr}}, \Phi_{e}$ and $W$ satisfy (2.1)-(2.4) and belong to the spaces

$$
\Phi_{\operatorname{tr}} \in L^{2}\left[(0, T), W^{2,2}(\Omega)\right] \cap W^{1,2}\left[(0, T), L^{2}(\Omega)\right] ;
$$

\footnotetext{
${ }^{6}$ Slightly modified from [4], p. 469, Definition 18.
} 


$$
\begin{aligned}
& \Phi_{e} \in L^{2}\left[(0, T), W^{2,2}(\Omega)\right] ; \\
& W \in W^{1,2}\left[(0, T), L^{2}(\Omega)\right]
\end{aligned}
$$

where $\int_{\Omega} \Phi_{e}(x, t) \mathrm{d} x=0$ holds for almost all $t \in(0, T)$.

2) (Weak solution of the bidomain system $)^{7}$ A triple $\left(\Phi_{\mathrm{tr}}, \Phi_{e}, W\right)$ is called a weak solution of the bidomain system (2.1)-(2.4) on [0,T] iff the functions $\Phi_{\mathrm{tr}}, \Phi_{e}$ and $W$ satisfy (2.1)-(2.4) and belong to the spaces

$$
\begin{aligned}
& \Phi_{\mathrm{tr}} \in C^{0}\left[[0, T], L^{2}(\Omega)\right] \cap L^{2}\left[(0, T), W^{1,2}(\Omega)\right] \cap L^{4}\left(\Omega_{T}\right) ; \\
& \Phi_{e} \in L^{2}\left[(0, T), W^{1,2}(\Omega)\right] \\
& W \in C^{0}\left[[0, T], L^{2}(\Omega)\right]
\end{aligned}
$$

where $\int_{\Omega} \Phi_{e}(x, t) \mathrm{d} x=0$ holds for almost all $t \in(0, T)$.

\subsection{Two-variable models for the ionic current}

For the ionic current $I_{\text {ion }}$ and the function $G$ within the gating equation, the following three models will be considered:

a) The Rogers-McCulloch model ${ }^{8}$.

$$
\begin{aligned}
& I_{\mathrm{ion}}(\varphi, w)=b \cdot \varphi(\varphi-a)(\varphi-1)+\varphi \cdot w=b \varphi^{3}-(a+1) b \varphi^{2}+a b \varphi+\varphi w \\
& G(\varphi, w)=\varepsilon w-\varepsilon \kappa \varphi
\end{aligned}
$$

with $0<a<1, b>0, \kappa>0$ and $\varepsilon>0$. Consequently, the gating variable obeys the linear ODE

$$
\partial W / \partial t+\varepsilon W=\varepsilon \kappa \Phi_{\mathrm{tr}} .
$$

b) The FitzHugh-Nagumo model ${ }^{9}$.

$$
\begin{aligned}
& I_{\text {ion }}(\varphi, w)=\varphi(\varphi-a)(\varphi-1)+w=\varphi^{3}-(a+1) \varphi^{2}+a \varphi+w \\
& G(\varphi, w)=\varepsilon w-\varepsilon \kappa \varphi
\end{aligned}
$$

with $0<a<1, \kappa>0$ and $\varepsilon>0$. Consequently, the gating variable obeys the same linear ODE (2.14) as before.

c) The linearized Aliev-Panfilov model ${ }^{10}$.

$$
\begin{aligned}
& I_{\mathrm{ion}}(\varphi, w)=b \cdot \varphi(\varphi-a)(\varphi-1)+\varphi \cdot w=b \varphi^{3}-(a+1) b \varphi^{2}+a b \varphi+\varphi w \\
& G(\varphi, w)=\varepsilon w-\varepsilon \kappa\left((a+1) \varphi-\varphi^{2}\right)
\end{aligned}
$$

with $0<a<1, b>0, \kappa>0$ and $\varepsilon>0$. The linear ODE for the gating variable is

$$
\partial W / \partial t+\varepsilon W=\varepsilon \kappa\left((a+1) \Phi_{\mathrm{tr}}-\Phi_{\mathrm{tr}}^{2}\right) .
$$

\footnotetext{
${ }^{7}[4]$, p. 472 , Definition 26.

${ }^{8}$ Introduced in [18].

${ }^{9}$ See [8] together with [17].

${ }^{10}$ The model, which appears to be a linearization of the original model derived in [2], is taken from [4], p. 480.
} 


\subsection{Existence and uniqueness of weak solutions; the stability estimate}

In [12], the following results about weak solutions of the bidomain system (2.1)-(2.4) have been obtained:

Theorem 2.3 (Existence and uniqueness of weak solutions). ${ }^{11}$ Assume that the data within (2.1)-(2.4) obey Assumptions 2.1., and specify the Rogers-McCulloch or the FitzHugh-Nagumo model. Then the bidomain system (2.1)-(2.4) admits for arbitrary initial values $\Phi_{0} \in L^{2}(\Omega), W_{0} \in L^{4}(\Omega)$ and inhomogeneities $I_{i}$, $I_{e} \in L^{\infty}\left[(0, T),\left(W^{1,2}(\Omega)\right)^{*}\right]$, which satisfy the compatibility condition

$$
\int_{\Omega}\left(I_{i}(x, t)+I_{e}(x, t)\right) \mathrm{d} x=0 \quad \text { for almost all } t \in(0, T),
$$

a uniquely determined weak solution $\left(\Phi_{\mathrm{tr}}, \Phi_{e}, W\right)$ on $[0, T]$ according to Definition 2.2, 2). If the linearized Aliev-Panfilov model is specified, this assertion remains true provided that $W_{0}$ belongs to $W^{1,3 / 2}(\Omega)$ instead of $L^{4}(\Omega)$.

In fact, a closer regularity analysis reveals that, under the assumptions of Theorem 2.3., the components $\left(\Phi_{\mathrm{tr}}, W\right)$ of a given weak solution of the bidomain system belong to $\left(L^{2}\left[(0, T), L^{6}(\Omega)\right] \cap L^{q}[(0, T)\right.$, $\left.\left.L^{r}(\Omega)\right]\right) \times C^{0}\left[[0, T], L^{4}(\Omega)\right]$ in the case of the Rogers-McCulloch or the FitzHugh-Nagumo model and to $\left(L^{2}\left[(0, T), L^{6}(\Omega)\right] \cap L^{q}\left[(0, T), L^{r}(\Omega)\right]\right) \times C^{0}\left[[0, T], L^{8 / 3}(\Omega)\right]$ in the case of the linearized AlievPanfilov model where $1<q<\infty$ and $4 \leqslant r<6$.

Theorem 2.4 (Stability estimate for weak solutions). ${ }^{12}$ Assume that the data within (2.1)-(2.4) obey Assumptions 2.1., and specify the Rogers-McCulloch or the FitzHugh-Nagumo model. Consider two weak solutions $\left(\Phi_{\mathrm{tr}}{ }^{\prime}, \Phi_{e}{ }^{\prime}, W^{\prime}\right),\left(\Phi_{\mathrm{tr}}{ }^{\prime \prime}, \Phi_{e}{ }^{\prime \prime}, W^{\prime \prime}\right)$ of $(2.1)-(2.4)$, which correspond to initial values $\Phi_{0}^{\prime}=\Phi_{0}^{\prime \prime}=\Phi_{0} \in L^{2}(\Omega)$, $W_{0}^{\prime}=W_{0}^{\prime \prime}=W_{0} \in L^{4}(\Omega)$ and inhomogeneities $I_{i}{ }^{\prime}, I_{e}{ }^{\prime}, I_{i}{ }^{\prime \prime}$ and $I_{e}{ }^{\prime \prime} \in L^{\infty}\left[(0, T),\left(W^{1,2}(\Omega)\right)^{*}\right]$ with

$$
\int_{\Omega}\left(I_{i}{ }^{\prime}(x, t)+I_{e}{ }^{\prime}(x, t)\right) \mathrm{d} x=\int_{\Omega}\left(I_{i}{ }^{\prime \prime}(x, t)+I_{e}{ }^{\prime \prime}(x, t)\right) \mathrm{d} x=0 \quad \text { for almost all } t \in(0, T),
$$

whose norms are bounded by $R>0$. Then the following estimate holds:

$$
\begin{aligned}
& \left\|\Phi_{\operatorname{tr}}{ }^{\prime}-\Phi_{\operatorname{tr}}{ }^{\prime \prime}\right\|_{L^{2}\left[(0, T), W^{1,2}(\Omega)\right]}^{2}+\left\|\Phi_{\operatorname{tr}}{ }^{\prime}-\Phi_{\operatorname{tr}}{ }^{\prime \prime}\right\|_{C^{0}\left[[0, T], L^{2}(\Omega)\right]}^{2} \\
& +\left\|\Phi_{\operatorname{tr}}{ }^{\prime}-\Phi_{\operatorname{tr}}{ }^{\prime \prime}\right\|_{W^{1,4 / 3}\left[(0, T),\left(W^{1,2}(\Omega)\right)^{*}\right]}+\left\|\Phi_{e}{ }^{\prime}-\Phi_{e}{ }^{\prime \prime}\right\|_{L^{2}\left[(0, T), W^{1,2}(\Omega)\right]}^{2} \\
& +\left\|W^{\prime}-W^{\prime \prime}\right\|_{L^{2}\left[(0, T), L^{2}(\Omega)\right]}^{2}+\left\|W^{\prime}-W^{\prime \prime}\right\|_{C^{0}\left[[0, T], L^{2}(\Omega)\right]}^{2}+\left\|W^{\prime}-W^{\prime \prime}\right\|_{W^{1,2}\left[(0, T), L^{2}(\Omega)\right]}^{2} \\
& \quad \leqslant C\left(\left\|I_{i}{ }^{\prime}-I_{i}{ }^{\prime \prime}\right\|_{L^{\infty}\left[(0, T),\left(W^{1,2}(\Omega)\right)^{*}\right]}^{2}+\left\|I_{e}{ }^{\prime}-I_{e}{ }^{\prime \prime}\right\|_{L^{\infty}\left[(0, T),\left(W^{1,2}(\Omega)\right)^{*}\right]}^{2}\right) .
\end{aligned}
$$

The constant $C>0$ does not depend on $I_{i}{ }^{\prime}, I_{e}{ }^{\prime}, I_{i}{ }^{\prime \prime}$ and $I_{e}{ }^{\prime \prime}$ but possibly on $\Omega, R, \Phi_{0}$ and $W_{0}$. If the linearized Aliev-Panfilov model is specified then the assertion remains true provided that $W_{0}^{\prime}=W_{0}^{\prime \prime}=W_{0}$ belong to $W^{1,3 / 2}(\Omega)$ instead of $L^{4}(\Omega)$.

The assumptions in Theorems 2.3.-2.4. are in accordance to the analytical framework wherein the control problem $(\mathrm{P})$ will be studied in the next sections.

\footnotetext{
${ }^{11}$ [4], p. 473, Theorem 30, together with [12], p. 8, Theorem 2.8, slightly modified. An error within the proof of this and the next theorem will be fixed in a subsequent publication.

12 [12], p. 7 f., Theorem 2.7 , slightly modified.
} 


\section{The optimal CONTRol PRoblem}

\subsection{Formulation of the problem within function spaces}

In order to provide a precise statement of the optimal control problem (1.7)-(1.8) within an appropriate function space framework, we introduce the following spaces:

$$
\begin{aligned}
& \mathrm{X}_{1}=L^{2}\left[(0, T), W^{1,2}(\Omega)\right] ; \quad \mathrm{X}_{2}=\mathrm{X}_{1} \cap\left\{Z \mid \int_{\Omega} Z(x, t) \mathrm{d} x=0(\forall) t \in(0, T)\right\} ; \\
& \mathrm{X}_{3}=L^{2}\left[(0, T), L^{2}(\Omega)\right] ; \quad \mathrm{X}_{4}=L^{\infty}\left[(0, T), L^{2}(\Omega)\right] .
\end{aligned}
$$

We will further specify the subspaces

$$
\begin{aligned}
& \widetilde{\mathrm{X}}_{1}=\mathrm{X}_{1} \cap W^{1,4 / 3}\left[(0, T),\left(W^{1,2}(\Omega)\right)^{*}\right] \cap C^{0}\left[[0, T], L^{2}(\Omega)\right] ; \quad \widetilde{\mathrm{X}}_{2}=\mathrm{X}_{2} ; \\
& \widetilde{\mathrm{X}}_{3}=\mathrm{X}_{3} \cap W^{1,2}\left[(0, T),\left(L^{2}(\Omega)\right)^{*}\right] \cap C^{0}\left[[0, T], L^{2}(\Omega)\right],
\end{aligned}
$$

which contain all polynomials and, consequently, lie dense in $\mathrm{X}_{1}, \mathrm{X}_{2}$ and $\mathrm{X}_{3}$, as well as the target spaces

$$
\begin{aligned}
& \mathrm{Z}_{1}=L^{4 / 3}\left[(0, T),\left(W^{1,2}(\Omega)\right)^{*}\right] ; \quad \mathrm{Z}_{2}=L^{2}\left[(0, T),\left(W^{1,2}(\Omega)\right)^{*}\right] ; \\
& \mathrm{Z}_{3}=L^{2}\left[(0, T),\left(L^{2}(\Omega)\right)^{*}\right] ; \quad \mathrm{Z}_{4}=\mathrm{Z}_{5}=L^{2}(\Omega) .
\end{aligned}
$$

The quadruples $\left(\Phi_{\mathrm{tr}}, \Phi_{e}, W, I_{e}\right)$ of state and control variables will be chosen from the space $\widetilde{\mathrm{X}}_{1} \times \widetilde{\mathrm{X}}_{2} \times \widetilde{\mathrm{X}}_{3} \times \mathrm{X}_{4}$. Recall the definition of $Q: L^{2}\left(\Omega_{T}\right) \rightarrow L^{2}\left(\Omega_{T}\right)$ as

$$
Q I(x, t)=I(x, t)-\mathbb{1}_{\Omega_{\text {con }}}(x) \cdot \frac{1}{\left|\Omega_{\text {con }}\right|} \int_{\Omega_{\text {con }}} I(\tilde{x}, t) \mathrm{d} \tilde{x} .
$$

With the aid of the operators

$$
\begin{aligned}
& F: \mathrm{X}_{1} \times \mathrm{X}_{2} \times \mathrm{X}_{3} \times \mathrm{X}_{4} \rightarrow \mathbb{R} ; \\
& E_{1}: \widetilde{\mathrm{X}}_{1} \times \widetilde{\mathrm{X}}_{2} \times \widetilde{\mathrm{X}}_{3} \rightarrow \mathrm{Z}_{1} ; \quad E_{2}: \widetilde{\mathrm{X}}_{1} \times \widetilde{\mathrm{X}}_{2} \times \mathrm{X}_{4} \rightarrow \mathrm{Z}_{2} ; \quad E_{3}: \widetilde{\mathrm{X}}_{1} \times \widetilde{\mathrm{X}}_{3} \rightarrow \mathrm{Z}_{3} ; \\
& E_{4}: \widetilde{\mathrm{X}}_{1} \rightarrow \mathrm{Z}_{4} ; \quad E_{5}: \widetilde{\mathrm{X}}_{3} \rightarrow \mathrm{Z}_{5},
\end{aligned}
$$

the problem $(\mathrm{P})$ will be restated now in the following way:

$$
\begin{aligned}
& \text { (P) } F\left(\Phi_{\mathrm{tr}}, \Phi_{e}, W, I_{e}\right)=\int_{0}^{T} \int_{\Omega} r\left(x, t, \Phi_{\operatorname{tr}}(x, t), \Phi_{e}(x, t), W(x, t)\right) \mathrm{d} x \mathrm{~d} t \\
& +\frac{\mu}{2} \cdot \int_{0}^{T} \int_{\Omega} I_{e}(x, t)^{2} \mathrm{~d} x \mathrm{~d} t \longrightarrow \inf ! \\
& E_{1}\left(\Phi_{\mathrm{tr}}, \Phi_{e}, W\right)=\mathfrak{o} \Longleftrightarrow \int_{\Omega}\left(\frac{\partial \Phi_{\mathrm{tr}}}{\partial t}+I_{\mathrm{ion}}\left(\Phi_{\mathrm{tr}}, W\right)\right) \psi \mathrm{d} x+\int_{\Omega} \nabla \psi^{\mathrm{T}} M_{i}\left(\nabla \Phi_{\mathrm{tr}}+\nabla \Phi_{e}\right) \mathrm{d} x=0 \\
& \forall \psi \in W^{1,2}(\Omega)(\forall) t \in(0, T) ; \\
& E_{2}\left(\Phi_{\mathrm{tr}}, \Phi_{e}, I_{e}\right)=\mathfrak{o} \Longleftrightarrow \int_{\Omega}\left(\nabla \psi^{\mathrm{T}} M_{i} \nabla \Phi_{\mathrm{tr}}+\nabla \psi^{\mathrm{T}}\left(M_{i}+M_{e}\right) \nabla \Phi_{e}\right) \mathrm{d} x-\int_{\Omega} I_{e} \psi \mathrm{d} x=0 \\
& \forall \psi \in W^{1,2}(\Omega) \text { with } \int_{\Omega} \psi(x) \mathrm{d} x=0(\forall) t \in(0, T) ; \\
& E_{3}\left(\Phi_{\mathrm{tr}}, W\right)=\mathfrak{o} \quad \Longleftrightarrow \int_{\Omega}\left(\frac{\partial W(t)}{\partial t}+G\left(\Phi_{\mathrm{tr}}(t), W(t)\right)\right) \psi \mathrm{d} x=0 \quad \forall \psi \in L^{2}(\Omega)(\forall) t \in(0, T) ;
\end{aligned}
$$




$$
\begin{aligned}
& E_{4}\left(\Phi_{\mathrm{tr}}\right)=\mathfrak{o} \Longleftrightarrow \Phi_{\operatorname{tr}}(x, 0)-\Phi_{0}(x)=0 \quad(\forall) x \in \Omega ; \\
& E_{5}(W)=\mathfrak{o} \Longleftrightarrow W(x, 0)-W_{0}(x)=0 \quad(\forall) x \in \Omega ; \\
& I_{e} \in \mathcal{C}=\left\{Q I \mid I \in L^{\infty}\left[(0, T), L^{2}(\Omega)\right], \operatorname{supp}(I) \subseteq \Omega_{\mathrm{con}} \times[0, T],\right. \\
& \left.|I(x, t)| \leqslant R \quad(\forall)(x, t) \in \Omega_{T}\right\} \subset L^{\infty}\left[(0, T), L^{2}(\Omega)\right] .
\end{aligned}
$$

Assumptions 2.1 are imposed on the data of problem (P). The numbers $T>0, \mu>0$ and $R>0$ as well as the Lipschitz subdomain $\Omega_{\text {con }} \subseteq \Omega$ are fixed. The functions $I_{\text {ion }}$ and $G$ will be specified according to any of the models from Section 2.2. In the case of the Rogers-McCulloch or the FitzHugh-Nagumo model, we fix initial values $\Phi_{0} \in L^{2}(\Omega)$ and $W_{0} \in L^{4}(\Omega)$ while in the case of the linearized Aliev-Panfilov model, $\Phi_{0} \in L^{2}(\Omega)$ and $W_{0} \in W^{1,3 / 2}(\Omega)$ will be used. Concerning the objective functional $F$, we assume the integrand

$$
r(x, t, \varphi, \eta, w): \Omega \times[0, T] \times \mathbb{R}^{3} \rightarrow \mathbb{R}
$$

to be measurable with respect to $x$ and $t$ and continuous with respect to $\varphi, \eta$ and $w$. With regard to (3.7) and (3.17), in the second term of $F$ the original integration domain $\Omega_{\text {con }}$ from (1.7) can be replaced by $\Omega$.

\subsection{Structure of the feasible domain}

Proposition 3.1. For the problem (3.11)-(3.17), the control-to-state-mapping $\mathcal{C} \ni I_{e} \longmapsto\left(\Phi_{\mathrm{tr}}, \Phi_{e}, W\right) \in \mathrm{X}_{1} \times$ $\mathrm{X}_{2} \times \mathrm{X}_{3}$ is well-defined.

Proof.

Recall that $\int_{\Omega} I_{e}(x, t) \mathrm{d} x=0$ for almost all $t \in(0, T)$. Consequently, the data within the problem (3.11)-(3.17) satisfy the assumptions of Theorem 2.3. with $I_{i}=\mathfrak{o}$, and the existence of a uniquely determined weak solution $\left(\Phi_{\mathrm{tr}}, \Phi_{e}, W\right)$ of the bidomain system is guaranteed for any feasible control $I_{e} \in \mathcal{C} \subset L^{\infty}\left[(0, T), L^{2}(\Omega)\right]$.

Proposition 3.2. The control domain $\mathcal{C} \subset L^{\infty}\left(\Omega_{T}\right)$ forms a closed, convex, weak*-sequentially compact subset of the space $\mathrm{X}_{4}$.

Proof.

Obviously, $\mathcal{C}$ is a convex subset of $\mathrm{X}_{4}$. In order to confirm closedness, consider a norm-convergent sequence $\left\{Q I^{N}\right\}$ with members in $\mathcal{C} \cap \mathrm{X}_{4}$ and limit element $\hat{I}$. Since the sequence $\left\{I^{N}\right\}$ of the generating functions is uniformly bounded in $L^{\infty}\left[(0, T), L^{2}\left(\Omega_{\text {con }}\right)\right]$, it admits a weak ${ }^{*}$-convergent subsequence $I^{N^{\prime}}$ with a limit element $\tilde{I}$ still satisfying the conditions $\operatorname{supp}(\tilde{I}) \subseteq \Omega_{\text {con }} \times[0, T]$ and $|\tilde{I}(x, t)| \leqslant R(\forall)(x, t) \in \Omega_{T}$. The weak*-continuity of the operator $Q$ implies then $Q I^{N^{\prime}} \stackrel{*}{\longrightarrow} \mathrm{X}_{4} Q \tilde{I}$ and $\hat{I}=Q \tilde{I} \in \mathcal{C}$. Now the weak*-sequential compactness of $\mathcal{C}$ is obtained from [19], (p. 301), Theorem VI.6.6. (together with p. 152), Theorem IV.4.11. Finally, $\|I\|_{L^{\infty}\left(\Omega_{T}\right)} \leqslant R$ implies $\|Q I\|_{L^{\infty}\left(\Omega_{T}\right)} \leqslant 2 R$, and $\mathcal{C}$ belongs even to $L^{\infty}\left(\Omega_{T}\right)$.

Proposition 3.3. The feasible domain $\mathcal{B}$ of the problem (3.11)-(3.17) is nonempty and closed with respect to the following topology in $\mathrm{X}_{1} \times \mathrm{X}_{2} \times \mathrm{X}_{3} \times \mathrm{X}_{4}$ : weak convergence with respect to the first three components, and weak $k^{*}$-convergence with respect to the fourth component.

Proof.

The existence of feasible solutions follows via Theorem 2.3 from Proposition 3.1. Consider now a sequence of feasible solutions $\left\{\left(\Phi_{\operatorname{tr}}{ }^{N}, \Phi_{e}{ }^{N}, W^{N}, I_{e}{ }^{N}\right)\right\}$ with $\Phi_{\operatorname{tr}}{ }^{N} \rightarrow \mathrm{X}_{1} \hat{\Phi}_{\mathrm{tr}}, \Phi_{e}{ }^{N} \rightarrow \mathrm{X}_{2} \hat{\Phi}_{e}, W^{N} \rightarrow \mathrm{X}_{3} \hat{W}$ and $I_{e}{ }^{N} \stackrel{*}{\rightarrow} \mathrm{X}_{4} \hat{I}_{e}$. From Proposition 3.2, we already know that $\hat{I}_{e}$ belongs to $\mathcal{C}$. Further, from [12], page 7, Theorem 2.6, we obtain uniform bounds with respect to $N$ for the norms of $\Phi_{\operatorname{tr}}{ }^{N}, \Phi_{e}{ }^{N}, W^{N}, \partial \Phi_{\operatorname{tr}}^{N} / \partial t$ and $\partial W^{N} / \partial t$, implying weak convergence of $\partial \Phi_{\operatorname{tr}}{ }^{N^{\prime}} / \partial t, \nabla \Phi_{\operatorname{tr}}{ }^{N^{\prime}}$ and $\partial W^{N^{\prime}} / \partial t$ as well as a.e. pointwise convergence of $\Phi_{\operatorname{tr}}{ }^{N^{\prime}}$ on $\Omega_{T}$ along a suitable subsequence. Consequently, passing to the limit $N^{\prime} \rightarrow \infty$ in (2.1)-(2.4), we may confirm that $\left(\hat{\Phi}_{\mathrm{tr}}, \hat{\Phi}_{e}, \hat{W}\right)$ solves the bidomain system with right-hand sides $I_{i}=\mathfrak{o}$ and $\hat{I}_{e}$. 


\subsection{Existence of global minimizers}

Theorem 3.4 (Existence of global minimizers in (P)). We impose the assumptions from Section 3.1. on the data of the problem (3.11)-(3.17). Assume further that the integrand $r(x, t, \varphi, \eta, w): \Omega \times[0, T] \times \mathbb{R}^{3} \rightarrow \mathbb{R}$ is bounded from below and convex with respect to $\varphi, \eta$ and $w$. Then the problem (3.11)-(3.17) admits a global minimizer.

Proof. Since $r$ is bounded from below, the problem (3.11)-(3.17) admits a minimizing sequence $\left\{\left(\Phi_{\operatorname{tr}}{ }^{N}\right.\right.$, $\left.\left.\Phi_{e}{ }^{N}, W^{N}, I_{e}{ }^{N}\right)\right\}$ of feasible solutions. Due to the uniform boundedness of $\left\|I_{e}{ }^{N}\right\|_{\mathrm{X}_{4}}$ with respect to $N$, the norms $\left\|\Phi_{\mathrm{tr}}{ }^{N}\right\|_{\mathrm{X}_{1}},\left\|\Phi_{e}{ }^{N}\right\|_{\mathrm{X}_{2}}$ and $\left\|W^{N}\right\|_{\mathrm{X}_{3}}$ are uniformly bounded as well (cf. again [12], p. 7, Thm. 2.6), and we may pass to a subsequence $\left\{\left(\Phi_{\operatorname{tr}}{ }^{N^{\prime}}, \Phi_{e}{ }^{N^{\prime}}, W^{N^{\prime}}, I_{e}{ }^{N^{\prime}}\right)\right\}$, which converges to a feasible quadruple $\left(\hat{\Phi}_{\mathrm{tr}}, \hat{\Phi}_{e}, \hat{W}, \hat{I}_{e}\right)$ in the sense of Proposition 3.3. The lower semicontinuity of the objective follows as in [6] (p. 96), Theorem 3.23, and page 97, Remark 3.25(ii). Consequently, denoting the minimal value of $(\mathrm{P})$ by $m$, we get

$$
\begin{aligned}
m=\lim _{N^{\prime} \rightarrow \infty} F\left(\Phi_{\mathrm{tr}}{ }^{\prime}, \Phi_{e}{ }^{N^{\prime}}, W^{N^{\prime}}, I_{e}{ }^{N^{\prime}}\right) & \\
& \geqslant \liminf _{N^{\prime} \rightarrow \infty} F\left(\Phi_{\mathrm{tr}}{ }^{N^{\prime}}, \Phi_{e}{ }^{N} p, W^{N^{\prime}}, I_{e}^{N^{\prime}}\right) \geqslant F\left(\hat{\Phi}_{\mathrm{tr}}, \hat{\Phi}_{e}, \hat{W}, \hat{I}_{e}\right) \geqslant m,
\end{aligned}
$$

and the quadruple $\left(\hat{\Phi}_{\mathrm{tr}}, \hat{\Phi}_{e}, \hat{W}, \hat{I}_{e}\right)$ is a global minimizer of $(\mathrm{P})$.

\section{The ADJoint EQUATIONS}

\subsection{Derivation of the adjoint system}

Throughout the following sections, we will further assume that the integrand $r(x, t, \varphi, \eta, w)$ within the objective (3.11) is continuously differentiable with respect to the variables $\varphi, \eta$ and $w$. For the optimal control problem $(\mathrm{P})$, let us introduce now the formal Lagrange function

$$
\begin{aligned}
\mathcal{L}\left(\Phi_{\mathrm{tr}}, \Phi_{e}, W, I_{e},\right. & \left.P_{1}, P_{2}, P_{3}, P_{4}, P_{5}\right)=F\left(\Phi_{\mathrm{tr}}, \Phi_{e}, W, I_{e}\right)+\left\langle P_{1}, E_{1}\left(\Phi_{\mathrm{tr}}, \Phi_{e}, W\right)\right\rangle \\
& +\left\langle P_{2}, E_{2}\left(\Phi_{\mathrm{tr}}, \Phi_{e}, I_{e}\right)\right\rangle+\left\langle P_{3}, E_{3}\left(\Phi_{\mathrm{tr}}, W\right)\right\rangle+\left\langle P_{4}, E_{4}\left(\Phi_{\mathrm{tr}}\right)\right\rangle+\left\langle P_{5}, E_{5}(W)\right\rangle
\end{aligned}
$$

with multipliers

$$
\begin{aligned}
& P_{1} \in L^{4}\left[(0, T), W^{1,2}(\Omega)\right] \\
& P_{2} \in L^{2}\left[(0, T), W^{1,2}(\Omega)\right] \cap\left\{Z \mid \int_{\Omega} Z(x, t) \mathrm{d} x=0(\forall) t \in(0, T)\right\} ; \\
& P_{3} \in L^{2}\left[(0, T), L^{2}(\Omega)\right] ; \quad P_{4}, P_{5} \in\left(L^{2}(\Omega)\right)^{*} .
\end{aligned}
$$

Differentiating $\mathcal{L}$ at the point $\left(\hat{\Phi}_{\mathrm{tr}}, \hat{\Phi}_{e}, \hat{W}, \hat{I}_{e}\right)$ in a formal way with respect to the variables $\Phi_{\mathrm{tr}}, \Phi_{e}$ and $W$, we find the adjoint equations

$$
\begin{aligned}
& D_{\Phi_{\mathrm{tr}}} F\left(\hat{\Phi}_{\mathrm{tr}}, \hat{\Phi}_{e}, \hat{W}, \hat{I}_{e}\right)+\left\langle P_{1}, D_{\Phi_{\mathrm{tr}}} E_{1}\left(\hat{\Phi}_{\mathrm{tr}}, \hat{\Phi}_{e}, \hat{W}\right)\right\rangle \\
&+\left\langle P_{2}, D_{\Phi_{\mathrm{tr}}} E_{2}\left(\hat{\Phi}_{\mathrm{tr}}, \hat{\Phi}_{e}, \hat{I}_{e}\right)\right\rangle+\left\langle P_{3}, D_{\Phi_{\mathrm{tr}}} E_{3}\left(\hat{\Phi}_{\mathrm{tr}}, \hat{W}\right)\right\rangle+\left\langle P_{4}, D_{\Phi_{\mathrm{tr}}} E_{4}\left(\hat{\Phi}_{\mathrm{tr}}\right)\right\rangle=0 ; \\
& D_{\Phi_{e}} F\left(\hat{\Phi}_{\mathrm{tr}}, \hat{\Phi}_{e}, \hat{W}, \hat{I}_{e}\right)+\left\langle P_{1}, D_{\Phi_{e}} E_{1}\left(\hat{\Phi}_{\mathrm{tr}}, \hat{\Phi}_{e}, \hat{W}\right)\right\rangle+\left\langle P_{2}, D_{\Phi_{e}} E_{2}\left(\hat{\Phi}_{\mathrm{tr}}, \hat{\Phi}_{e}, \hat{I}_{e}\right)\right\rangle=0 \\
& D_{W} F\left(\hat{\Phi}_{\mathrm{tr}}, \hat{\Phi}_{e}, \hat{W}, \hat{I}_{e}\right)+\left\langle P_{1}, D_{W} E_{1}\left(\hat{\Phi}_{\mathrm{tr}}, \hat{\Phi}_{e}, \hat{W}\right)\right\rangle+\left\langle P_{3}, D_{W} E_{3}\left(\hat{\Phi}_{\mathrm{tr}}, \hat{W}\right)\right\rangle+\left\langle P_{5}, D_{W} E_{5}(\hat{W})\right\rangle=0 .
\end{aligned}
$$


After choosing $P_{4}=-P_{1}(\cdot, 0)$ and $P_{5}=-P_{3}(\cdot, 0)$ (this choice is possible by Theorem 4.2. below), the adjoint system takes the following form:

$$
\begin{gathered}
\int_{0}^{T} \int_{\Omega}\left(-\frac{\partial P_{1}}{\partial t}+\frac{\partial I_{\mathrm{ing}}}{\partial \varphi}\left(\hat{\Phi}_{\mathrm{tr}}, \hat{W}\right) P_{1}+\frac{\partial G}{\partial \varphi}\left(\hat{\Phi}_{\mathrm{tr}}, \hat{W}\right) P_{3}\right) \psi \mathrm{d} x \mathrm{~d} t+\int_{0}^{T} \int_{\Omega} \nabla \psi^{\mathrm{T}} M_{i}\left(\nabla P_{1}+\nabla P_{2}\right) \mathrm{d} x \mathrm{~d} t \\
=-\int_{0}^{T} \int_{\Omega}\left(\frac{\partial r}{\partial \varphi}\left(\hat{\Phi}_{\mathrm{tr}}, \hat{\Phi}_{e}, \hat{W}\right)\right) \psi \mathrm{d} x \mathrm{~d} t \quad \forall \psi \in L^{2}\left[(0, T), W^{1,2}(\Omega)\right], P_{1}(x, T) \equiv 0 ; \\
\int_{0}^{T} \int_{\Omega} \nabla \psi^{\mathrm{T}} M_{i} \nabla P_{1} \mathrm{~d} x \mathrm{~d} t+\int_{0}^{T} \int_{\Omega} \nabla \psi^{\mathrm{T}}\left(M_{i}+M_{e}\right) \nabla P_{2} \mathrm{~d} x \mathrm{~d} t=-\int_{0}^{T} \int_{\Omega} \frac{\partial r}{\partial \eta}\left(\hat{\Phi}_{\mathrm{tr}}, \hat{\Phi}_{e}, \hat{W}\right) \psi \mathrm{d} x \mathrm{~d} t \\
\forall \psi \in L^{2}\left[(0, T), W^{1,2}(\Omega)\right] \text { with } \int_{\Omega} \psi(x, t) \mathrm{d} x=0 \quad(\forall) t \in(0, T), \int_{\Omega} P_{2}(x, t) \mathrm{d} x=0 \quad(\forall) t \in(0, T) ; \\
\int_{0}^{T} \int_{\Omega}\left(-\frac{\partial P_{3}}{\partial t}+\frac{\partial I_{\mathrm{ion}}}{\partial w}\left(\hat{\Phi}_{\mathrm{tr}}, \hat{W}\right) P_{1}+\frac{\partial G}{\partial w}\left(\hat{\Phi}_{\mathrm{tr}}, \hat{W}\right) P_{3}\right) \psi \mathrm{d} x \mathrm{~d} t=-\int_{0}^{T} \int_{\Omega}\left(\frac{\partial r}{\partial w}\left(\hat{\Phi}_{\mathrm{tr}}, \hat{\Phi}_{e}, \hat{W}\right)\right) \psi \mathrm{d} x \mathrm{~d} t(4.10) \\
\forall \psi \in L^{2}\left[(0, T), L^{2}(\Omega)\right], P_{3}(x, T) \equiv 0 .
\end{gathered}
$$

\subsection{The reduced form of the adjoint system}

First, we apply to the system (4.8)-(4.10) the transformation $s=T-t$, thus defining $\widetilde{P}_{i}(x, s)=P_{i}(x, T-s)$, $1 \leqslant i \leqslant 3, \widetilde{\Phi_{\operatorname{tr}}}(x, s)=\hat{\Phi}_{\operatorname{tr}}(x, T-s), \widetilde{\Phi}(x, s)=\hat{\Phi}_{e}(x, T-s), \widetilde{W}(x, s)=\hat{W}(x, T-s)$ and $\widetilde{I}_{e}(x, s)=\hat{I}_{e}(x, T-s)$ etc. By abuse of notation, we suppress all tildes, thus simply replacing $t$ by $s$ and $-\partial P_{1} / \partial t,-\partial P_{3} / \partial t$ by $\partial P_{1} / \partial s$ and $\partial P_{3} / \partial s$, respectively. Then the adjoint system, in analogy to the primal bidomain equations, can be rewritten in terms of the bidomain bilinear form as a reduced system:

$$
\begin{aligned}
& \frac{\mathrm{d}}{\mathrm{d} s}\left\langle P_{1}(s), \psi\right\rangle+A\left(P_{1}(s), \psi\right)+\int_{\Omega}\left(\frac{\partial I_{\mathrm{ion}}}{\partial \varphi}\left(\hat{\Phi}_{\mathrm{tr}}, \hat{W}\right) P_{1}+\frac{\partial G}{\partial \varphi}\left(\hat{\Phi}_{\mathrm{tr}}, \hat{W}\right) P_{3}\right) \psi \mathrm{d} x=\langle\widetilde{S}(s), \psi\rangle \\
& \forall \psi \in W^{1,2}(\Omega) ; \\
& \frac{\mathrm{d}}{\mathrm{d} s}\left\langle P_{3}(s), \psi\right\rangle+\int_{\Omega}\left(\frac{\partial I_{\mathrm{ion}}}{\partial w}\left(\hat{\Phi}_{\mathrm{tr}}, \hat{W}\right) P_{1}+\frac{\partial G}{\partial w}\left(\hat{\Phi}_{\mathrm{tr}}, \hat{W}\right) P_{3}\right) \psi \mathrm{d} x=-\left\langle\frac{\partial r}{\partial w}\left(\hat{\Phi}_{\mathrm{tr}}, \hat{\Phi}_{e}, \hat{W}\right), \psi\right\rangle \\
& \begin{array}{lll}
P_{1}(x, 0)=0 & (\forall) x \in \Omega ; & (4.12) \\
P_{3}(x, 0)=0 & (\forall) x \in \Omega
\end{array}
\end{aligned}
$$

on $[0, T]$ in distributional sense (cf. [12], p. 5f.), Theorem 2.4. Here the bidomain bilinear form $A$ : $W^{1,2}(\Omega) \times$ $W^{1,2}(\Omega) \rightarrow \mathbb{R}$ is defined as ibid., page $5,(2.22)$, through

$$
A\left(\psi_{1}, \psi_{2}\right)=\int_{\Omega} \nabla \psi_{1}^{\mathrm{T}} M_{i} \nabla \psi_{2} \mathrm{~d} x+\int_{\Omega} \nabla \widetilde{\psi}_{e}^{\mathrm{T}} M_{i} \nabla \psi_{2} \mathrm{~d} x
$$

where $\widetilde{\psi}_{e} \in W^{1,2}(\Omega)$ is the uniquely determined solution of the variational equation

$$
\begin{array}{r}
\int_{\Omega} \nabla \widetilde{\psi}_{e}^{\mathrm{T}}\left(M_{i}+M_{e}\right) \nabla \psi \mathrm{d} x=-\int_{\Omega} \nabla \psi_{1}^{\mathrm{T}} M_{i} \nabla \psi \mathrm{d} x \quad \forall \psi \in W^{1,2}(\Omega) \text { with } \int_{\Omega} \psi \mathrm{d} x=0 \quad \\
\text { satisfying } \int_{\Omega} \widetilde{\psi}_{e} \mathrm{~d} x=0,
\end{array}
$$

and the linear functionals $\widetilde{S}(s) \in\left(W^{1,2}(\Omega)\right)^{*}$ are defined through

$$
\langle\widetilde{S}(s), \psi\rangle=-\left\langle\frac{\partial r}{\partial \varphi}\left(\hat{\Phi}_{\mathrm{tr}}, \hat{\Phi}_{e}, \hat{W}\right), \psi\right\rangle-\int_{\Omega} \nabla \bar{\psi}_{e}^{\mathrm{T}} M_{i} \nabla \psi \mathrm{d} x
$$


where $\bar{\psi}_{e} \in W^{1,2}(\Omega)$ is the uniquely determined solution of the variational equation

$$
\begin{array}{r}
\int_{\Omega} \nabla \bar{\psi}_{e}^{\mathrm{T}}\left(M_{i}+M_{e}\right) \nabla \psi \mathrm{d} x=\left\langle\frac{\partial r}{\partial \eta}\left(\hat{\Phi}_{\mathrm{tr}}, \hat{\Phi}_{e}, \hat{W}\right), \psi\right\rangle \quad \forall \psi \in W^{1,2}(\Omega) \text { with } \int_{\Omega} \psi \mathrm{d} x=0 \quad \text { satisfying } \int_{\Omega} \bar{\psi}_{e} \mathrm{~d} x=0 . \\
\text { s.17) }
\end{array}
$$

The component $P_{2}$ of the solution of (4.8)-(4.10) is uniquely determined as the sum $P_{2}=\widetilde{\psi}_{e}+\bar{\psi}_{e}$. Note that this reformulation is even possible without imposing the additional compatibility condition

$$
\int_{\Omega} \frac{\partial r}{\partial \eta}\left(\hat{\Phi}_{\operatorname{tr}}(x, s), \hat{\Phi}_{e}(x, s), \hat{W}(x, s)\right) \mathrm{d} x=0 \quad(\forall) s \in(0, T) .
$$

\subsection{Existence and regularity of weak solutions}

Theorem 4.1 (A priori estimates for weak solutions of the adjoint system). The optimal control problem (3.11)-(3.17) is studied under the assumptions from Section 3.1. Within the problem, we specify the RogersMcCulloch model. Assume further that the integrand $r(x, t, \varphi, \eta, w)$ is continuously differentiable with respect to $\varphi, \eta$ and $w$.

1) If $\left(\hat{\Phi}_{\mathrm{tr}}, \hat{\Phi}_{e}, \hat{W}, \hat{I}_{e}\right)$ is a feasible solution of $(\mathrm{P})$ with

$$
\frac{\partial r}{\partial \varphi}\left(\hat{\Phi}_{\mathrm{tr}}, \hat{\Phi}_{e}, \hat{W}\right), \frac{\partial r}{\partial \eta}\left(\hat{\Phi}_{\mathrm{tr}}, \hat{\Phi}_{e}, \hat{W}\right), \frac{\partial r}{\partial w}\left(\hat{\Phi}_{\mathrm{tr}}, \hat{\Phi}_{e}, \hat{W}\right) \in L^{2}\left(\Omega_{T}\right)
$$

then every weak solution $\left(P_{1}, P_{2}, P_{3}\right) \in L^{2}\left[(0, T), W^{1,2}(\Omega)\right] \times L^{2}\left[(0, T), W^{1,2}(\Omega)\right] \times L^{2}\left(\Omega_{T}\right)$ of the adjoint system (4.8)-(4.10) obeys the estimate

$$
\begin{aligned}
& \left.\left\|P_{1}\right\|_{L^{\infty}\left[(0, T), L^{2}(\Omega)\right]}^{2}+\left\|P_{1}\right\|_{L^{2}\left[(0, T), W^{1,2}(\Omega)\right]}^{2}+\left\|P_{2}\right\|_{L^{2}\left[(0, T), W^{1,2}(\Omega)\right]}^{2}+\left\|P_{3}\right\|_{L^{\infty}\left[(0, T), L^{2}(\Omega)\right]}^{2}\right] \\
& \leqslant C\left(\left\|\frac{\partial r}{\partial \varphi}\left(\hat{\Phi}_{\mathrm{tr}}, \hat{\Phi}_{e}, \hat{W}\right)\right\|_{L^{2}\left(\Omega_{T}\right)}^{2}+\left\|\frac{\partial r}{\partial \eta}\left(\hat{\Phi}_{\mathrm{tr}}, \hat{\Phi}_{e}, \hat{W}\right)\right\|\left\|_{L^{2}\left(\Omega_{T}\right)}^{2}+\right\| \frac{\partial r}{\partial w}\left(\hat{\Phi}_{\mathrm{tr}}, \hat{\Phi}_{e}, \hat{W}\right) \|_{L^{2}\left(\Omega_{T}\right)}^{2}\right)
\end{aligned}
$$

where the constant $C>0$ does not depend on $P_{1}, P_{2}, P_{3}$ but on $\left(\hat{\Phi}_{\mathrm{tr}}, \hat{\Phi}_{e}, \hat{W}, \hat{I}_{e}\right)$ and the data of $(\mathrm{P})$.

2) Let $q=10 / 9$. If $\left(\hat{\Phi}_{\mathrm{tr}}, \hat{\Phi}_{e}, \hat{W}, \hat{I}_{e}\right)$ is a feasible solution of $(\mathrm{P})$ with

$$
\frac{\partial r}{\partial \varphi}\left(\hat{\Phi}_{\mathrm{tr}}, \hat{\Phi}_{e}, \hat{W}\right), \frac{\partial r}{\partial \eta}\left(\hat{\Phi}_{\mathrm{tr}}, \hat{\Phi}_{e}, \hat{W}\right), \frac{\partial r}{\partial w}\left(\hat{\Phi}_{\mathrm{tr}}, \hat{\Phi}_{e}, \hat{W}\right) \in L^{2 q}\left[(0, T), L^{2}(\Omega)\right]
$$

then every weak solution $\left(P_{1}, P_{2}, P_{3}\right) \in L^{2}\left[(0, T), W^{1,2}(\Omega)\right] \times L^{2}\left[(0, T), W^{1,2}(\Omega)\right] \times L^{2}\left(\Omega_{T}\right)$ of the adjoint system (4.8)-(4.10) obeys (4.20) as well as the further estimate

$$
\begin{aligned}
\left\|P_{1}\right\|_{C^{0}\left[[0, T], L^{2}(\Omega)\right]}^{2}+\left\|\partial P_{1} / \partial s\right\|_{L^{q}\left[(0, T),\left(W^{1,2}(\Omega)\right)^{*}\right]}^{q}+\left\|P_{3}\right\|_{C^{0}\left[[0, T], L^{2}(\Omega)\right]}^{2}\left[\left\|\frac{\partial r}{\partial \varphi}\left(\hat{\Phi}_{\mathrm{tr}}, \hat{\Phi}_{e}, \hat{W}\right)\right\|_{L^{2 q}\left[(0, T), L^{2}(\Omega)\right]}^{2 q}\right. \\
+\left\|\partial P_{3} / \partial s\right\|_{L^{q}\left[(0, T), L^{2}(\Omega)\right]}^{q} \leqslant\left(1+\left\|\frac{\partial r}{\partial w}\left(\hat{\Phi}_{\mathrm{tr}}, \hat{\Phi}_{e}, \hat{W}\right)\right\|_{L^{2}\left(\Omega_{T}\right)}^{2}\right) \\
\left.+\left\|\frac{\partial r}{\partial \eta}\left(\hat{\Phi}_{\mathrm{tr}}, \hat{\Phi}_{e}, \hat{W}\right)\right\|_{L^{2 q}\left[(0, T), L^{2}(\Omega)\right]}^{2 q}+\| \frac{1}{\partial{ }^{2}}\right)
\end{aligned}
$$

where the constant $C>0$ does not depend on $P_{1}, P_{2}, P_{3}$ but on $\left(\hat{\Phi}_{\mathrm{tr}}, \hat{\Phi}_{e}, \hat{W}, \hat{I}_{e}\right)$ and the data of $(\mathrm{P})$. 
The a priori estimates yield the following existence and uniqueness theorem for the adjoint system:

Theorem 4.2 (Existence and uniqueness of weak solutions for the adjoint system).

Under the assumptions of Theorem 4.1, 2), the adjoint system (4.8)-(4.10) admits a uniquely determined weak solution $\left(P_{1}, P_{2}, P_{3}\right)$ with

$$
\begin{aligned}
& P_{1} \in C^{0}\left[[0, T], L^{2}(\Omega)\right] \cap L^{2}\left[(0, T), W^{1,2}(\Omega)\right] \cap W^{1, q}\left[(0, T),\left(W^{1,2}(\Omega)\right)^{*}\right] ; \\
& P_{2} \in L^{2}\left[(0, T), W^{1,2}(\Omega)\right] ; \quad \int_{\Omega} P_{2}(x, t) \mathrm{d} x=0(\forall) t \in(0, T) ; \\
& P_{3} \in C^{0}\left[[0, T], L^{2}(\Omega)\right] \cap W^{1, q}\left[(0, T), L^{2}(\Omega)\right] .
\end{aligned}
$$

Note that, even under the assumptions of Theorems 4.1,2) and 4.2, the regularity of $P_{1} \in L^{4}\left[(0, T), W^{1,2}(\Omega)\right]$ as required in (4.1) and (4.2) cannot be guaranteed.

\subsection{Proofs}

Proof of Theorem 4.1. Throughout the proof, $C$ denotes a generical positive constant, which may appropriately change from line to line. Further, we will specify in (3.12)-(3.14) the Rogers-McCulloch model. The necessary alterations in the case of the other models will be discussed at the end of the subsection.

- Step 1. An estimate for the right-hand side of (4.11). We start with

Lemma 4.3. Under the assumptions of Theorem 4.1., for arbitrary $\varepsilon_{0}^{\prime}>0$ the following estimate holds:

$$
|\langle\widetilde{S}(s), \psi\rangle| \leqslant \frac{C}{\varepsilon_{0}^{\prime}}\left(\left\|\frac{\partial r}{\partial \varphi}\left(\hat{\Phi}_{\mathrm{tr}}, \hat{\Phi}_{e}, \hat{W}\right)\right\|_{L^{2}(\Omega)}^{2}+\left\|\frac{\partial r}{\partial \eta}\left(\hat{\Phi}_{\mathrm{tr}}, \hat{\Phi}_{e}, \hat{W}\right)\right\|{ }_{L^{2}(\Omega)}\right)+C \varepsilon_{0}^{\prime}\|\psi\|_{W^{1,2}(\Omega)}^{2} .
$$

The constant $C>0$ does not depend on $\varepsilon_{0}^{\prime}$ and $\psi$.

Proof. Inserting $\bar{\psi}_{e} \in W^{1,2}(\Omega)$ as a feasible test function into (4.17), we get from the uniform ellipticity of $M_{i}$ and $M_{e}$ and the Poincaré inequality:

$$
\begin{aligned}
C\left\|\bar{\psi}_{e}\right\|_{W^{1,2}(\Omega)}^{2} \leqslant & \int_{\Omega} \nabla \bar{\psi}_{e}^{\mathrm{T}}\left(M_{i}+M_{e}\right) \nabla \bar{\psi}_{e}^{\mathrm{T}} \mathrm{d} x \leqslant\left|\left\langle\frac{\partial r}{\partial \eta}\left(\hat{\Phi}_{\mathrm{tr}}, \hat{\Phi}_{e}, \hat{W}\right), \bar{\psi}_{e}^{\mathrm{T}}\right\rangle\right| \\
& \left.\leqslant \frac{1}{2 \delta_{1}}\left\|\frac{\partial r}{\partial \eta}\left(\hat{\Phi}_{\mathrm{tr}}, \hat{\Phi}_{e}, \hat{W}\right)\right\|_{\left(W^{1,2}(\Omega)\right.}^{2}\right)^{*}+\frac{\delta_{1}}{2}\left\|\bar{\psi}_{e}\right\|_{W^{1,2}(\Omega)}^{2} \quad(\forall) s \in(0, T),
\end{aligned}
$$

for arbitrary $\delta_{1}>0$. Inserting $\delta_{1}=C$, we arrive at

$$
\frac{C}{2}\left\|\bar{\psi}_{e}\right\|_{W^{1,2}(\Omega)}^{2} \leqslant \frac{1}{2 C}\left\|\frac{\partial r}{\partial \eta}\left(\hat{\Phi}_{\mathrm{tr}}, \hat{\Phi}_{e}, \hat{W}\right)\right\|_{\left(W^{1,2}(\Omega)\right.}^{2} \leqslant \frac{1}{2 C}\left\|\frac{\partial r}{\partial \eta}\left(\hat{\Phi}_{\mathrm{tr}}, \hat{\Phi}_{e}, \hat{W}^{2}\right)\right\|_{L^{2}(\Omega)}^{2} .
$$

From (4.16), we obtain

$$
\begin{aligned}
& |\langle\widetilde{S}(s), \psi\rangle| \leqslant\left|\left\langle\frac{\partial r}{\partial \varphi}\left(\hat{\Phi}_{\mathrm{tr}}, \hat{\Phi}_{e}, \hat{W}\right), \psi\right\rangle\right|+\left|\left\langle\nabla \bar{\psi}_{e}^{\mathrm{T}} M_{i}, \nabla \psi\right\rangle\right| \\
& \left.\leqslant \frac{1}{2 \delta_{2}}\left\|\frac{\partial r}{\partial \varphi}\left(\hat{\Phi}_{\mathrm{tr}}, \hat{\Phi}_{e}, \hat{W}\right)\right\| \|_{\left(W^{1,2}(\Omega)\right.}^{2}\right)^{*}+\frac{\delta_{2}}{2}\|\psi\|_{W^{1,2}(\Omega)}^{2}+\frac{1}{2 \delta_{3}}\left\|\bar{\psi}_{e}\right\|_{L^{2}(\Omega)}^{2}+\frac{\delta_{3}}{2}\left\|M_{i}\right\|^{2} \cdot\|\psi\|_{L^{2}(\Omega)}^{2} \\
& \leqslant \frac{1}{2 \delta_{2}}\left\|\frac{\partial r}{\partial \varphi}\left(\hat{\Phi}_{\mathrm{tr}}, \hat{\Phi}_{e}, \hat{W}\right)\right\|_{L^{2}(\Omega)}^{2}+\frac{1}{2 \delta_{3}}\left\|\bar{\psi}_{e}\right\|_{W^{1,2}(\Omega)}^{2}+\left(\frac{\delta_{2}}{2}+\frac{\left(\mu_{2}\right)^{2} \delta_{3}}{2}\right)\|\psi\|_{W^{1,2}(\Omega)}^{2} \\
& \leqslant \frac{C}{\delta_{2}}\left\|\frac{\partial r}{\partial \varphi}\left(\hat{\Phi}_{\mathrm{tr}}, \hat{\Phi}_{e}, \hat{W}\right)\right\|_{L^{2}(\Omega)}^{2}+\frac{C}{\delta_{3}}\left\|\frac{\partial r}{\partial \eta}\left(\hat{\Phi}_{\mathrm{tr}}, \hat{\Phi}_{e}, \hat{W}\right)\right\|{ }_{L^{2}(\Omega)}^{2}+C\left(\frac{\delta_{2}}{2}+\frac{\left(\mu_{2}\right)^{2} \delta_{3}}{2}\right)\|\psi\|_{W^{1,2}(\Omega)}^{2}
\end{aligned}
$$

by (2.5) and (4.28). Taking $\delta_{3}=\delta_{2} /\left(\mu_{2}\right)^{2}$, we get (4.26). 
- Step 2. The estimates for $\left\|P_{1}\right\|_{L^{\infty}\left[[0, T], L^{2}(\Omega)\right]}$ and $\left.\left\|P_{3}\right\|_{L^{\infty}\left[[0, T], L^{2}(\Omega)\right.}\right]$. Specifying the derivatives of $I_{\text {ion }}$ and $G$ according to the Rogers-McCulloch model, we have

$$
\begin{aligned}
& \frac{\partial I_{\mathrm{ion}}}{\partial \varphi}\left(\hat{\Phi}_{\mathrm{tr}}, \hat{W}\right)=3 b\left(\hat{\Phi}_{\mathrm{tr}}\right)^{2}-2(a+1) b \hat{\Phi}_{\mathrm{tr}}+a b+\hat{W} ; \quad \frac{\partial G}{\partial \varphi}\left(\hat{\Phi}_{\mathrm{tr}}, \hat{W}\right) \equiv-\varepsilon \kappa ; \\
& \frac{\partial I_{\mathrm{ion}}}{\partial w}\left(\hat{\Phi}_{\mathrm{tr}}, \hat{W}\right)=\hat{\Phi}_{\mathrm{tr}} ; \quad \frac{\partial G}{\partial w}\left(\hat{\Phi}_{\mathrm{tr}}, \hat{W}\right) \equiv \varepsilon .
\end{aligned}
$$

Inserting $P_{1}(s)$ as a feasible test function into (4.11), we get for arbitrary $\varepsilon_{0}^{\prime}, \varepsilon_{1}(s)>0$ with [12], page 6 , Theorem 2.4, 2), and Lemma 4.3 above $^{13}$

$$
\begin{aligned}
& \frac{1}{2} \frac{\mathrm{d}}{\mathrm{d} s}\left\|P_{1}(s)\right\|_{L^{2}(\Omega)}^{2}+\left(A\left(P_{1}, P_{1}\right)+\beta\left\|P_{1}\right\|_{L^{2}(\Omega)}^{2}\right)+\int_{\Omega} \frac{\partial I_{\mathrm{ion}}}{\partial \varphi}\left(\hat{\Phi}_{\mathrm{tr}}, \hat{W}\right) P_{1}(s)^{2} \mathrm{~d} x \\
& \quad \leqslant \int_{\Omega}\left|\frac{\partial G}{\partial \varphi}\left(\hat{\Phi}_{\mathrm{tr}}, \hat{W}\right)\right|\left|P_{1} P_{3}\right|+\left|\left\langle\widetilde{S}(s), P_{1}\right\rangle\right|+\beta\left\|P_{1}\right\|_{L^{2}(\Omega)}^{2} \Longrightarrow \\
& \frac{1}{2} \frac{\mathrm{d}}{\mathrm{d} s}\left\|P_{1}(s)\right\|_{L^{2}(\Omega)}^{2}+\beta\left\|P_{1}\right\|_{W^{1,2}(\Omega)}^{2}+\int_{\Omega}\left(a b+3 b\left(\hat{\Phi}_{\mathrm{tr}}\right)^{2}\right) P_{1}(s)^{2} \mathrm{~d} x \\
& \quad \leqslant C \int_{\Omega}\left(\left|\hat{\Phi}_{\mathrm{tr}}\right|+|\hat{W}|\right)\left|P_{1}\right|^{2} \mathrm{~d} x+\varepsilon \kappa \int_{\Omega}\left|P_{1} P_{3}\right| \mathrm{d} x \\
& \quad+\frac{C}{\varepsilon_{0}^{\prime}}\left(\left\|\frac{\partial r}{\partial \varphi}\left(\hat{\Phi}_{\mathrm{tr}}, \hat{\Phi}_{e}, \hat{W}\right)\right\|_{L^{2}(\Omega)}^{2}+\left\|\frac{\partial r}{\partial \eta}\left(\hat{\Phi}_{\mathrm{tr}}, \hat{\Phi}_{e}, \hat{W}\right)\right\|_{L^{2}(\Omega)}^{2}\right)+C \varepsilon_{0}^{\prime}\left\|P_{1}\right\|_{W^{1,2}(\Omega)}^{2}+\beta\left\|P_{1}\right\|_{L^{2}(\Omega)}^{2} \\
& \frac{1}{2} \frac{\mathrm{d}}{\mathrm{d} s}\left\|P_{1}(s)\right\|_{L^{2}(\Omega)}^{2}+\beta\left\|P_{1}\right\|_{W^{1,2}(\Omega)}^{2} \\
& \quad \leqslant C \varepsilon_{1}(s) \int_{\Omega}\left(\left|\hat{\Phi}_{\mathrm{tr}}\right|^{2}+|\hat{W}|^{2}\right)\left|P_{1}\right|^{2} \mathrm{~d} x+\frac{C}{\varepsilon_{1}(s)}\left\|P_{1}\right\|_{L^{2}(\Omega)}^{2}+C\left(\left\|P_{1}\right\|_{L^{2}(\Omega)}^{2}+\left\|P_{3}\right\|_{L^{2}(\Omega)}^{2}\right) \\
& \quad+\frac{C}{\varepsilon_{0}^{\prime}}\left(\left\|\frac{\partial r}{\partial \varphi}\left(\hat{\Phi}_{\mathrm{tr}}, \hat{\Phi}_{e}, \hat{W}\right)\right\|_{L^{2}(\Omega)}^{2}+\left\|\frac{\partial r}{\partial \eta}\left(\hat{\Phi}_{\mathrm{tr}}, \hat{\Phi}_{e}, \hat{W}\right)\right\|_{L^{2}(\Omega)}^{2}\right)+C \varepsilon_{0}^{\prime}\left\|P_{1}\right\|_{W^{1,2}(\Omega)}^{2}+\beta\left\|P_{1}\right\|_{L^{2}(\Omega)}^{2} \\
& \quad \leqslant C \varepsilon_{1}(s)\left(\left\|\hat{\Phi}_{\mathrm{tr}}\right\|_{L^{4}(\Omega)}^{2}+\| \hat{W}_{L^{4}(\Omega)}^{2}\right) \cdot\left\|P_{1}\right\|_{W^{1,2}(\Omega)}^{2}+C\left(1+\frac{1}{\varepsilon_{1}(s)}\right)\left\|P_{1}\right\|_{L^{2}(\Omega)}^{2} \\
& \quad+\frac{C}{\varepsilon_{0}^{\prime}}\left(\left\|\frac{\partial r}{\partial \varphi}\left(\hat{\Phi}_{\mathrm{tr}}, \hat{\Phi}_{e}, \hat{W}\right)\right\|_{L^{2}(\Omega)}^{2}+\left\|\frac{\partial r}{\partial \eta}\left(\hat{\Phi}_{\mathrm{tr}}, \hat{\Phi}_{e}, \hat{W}\right)\right\|_{L^{2}(\Omega)}^{2}\right)+C \varepsilon_{0}^{\prime}\left\|P_{1}\right\|_{W^{1,2}(\Omega)}^{2}+C\left\|P_{3}\right\|_{L^{2}(\Omega)}^{2} .
\end{aligned}
$$

We choose $\varepsilon_{1}(s)=\varepsilon_{1}^{\prime} /\left(1+\left\|\hat{\Phi}_{\mathrm{tr}}(s)\right\|_{L^{4}(\Omega)}^{2}+\|\hat{W}(s)\|_{L^{4}(\Omega)}^{2}\right)$ with $\varepsilon_{1}^{\prime}>0$ and continue (4.38) with

$$
\begin{aligned}
& \ldots \leqslant C \varepsilon_{1}^{\prime} \cdot\left\|P_{1}\right\|_{W^{1,2}(\Omega)}^{2}+C\left(1+\frac{1}{\varepsilon_{1}^{\prime}}\left(1+\left\|\hat{\Phi}_{\mathrm{tr}}(s)\right\|_{L^{4}(\Omega)}^{2}+\|\hat{W}(s)\|_{L^{4}(\Omega)}^{2}\right)\right) \cdot\left\|P_{1}\right\|_{L^{2}(\Omega)}^{2} \\
& +\frac{C}{\varepsilon_{0}^{\prime}}\left(\left\|\frac{\partial r}{\partial \varphi}\left(\hat{\Phi}_{\mathrm{tr}}, \hat{\Phi}_{e}, \hat{W}\right)\right\|_{L^{2}(\Omega)}^{2}+\left\|\frac{\partial r}{\partial \eta}\left(\hat{\Phi}_{\mathrm{tr}}, \hat{\Phi}_{e}, \hat{W}\right)\right\|_{L^{2}(\Omega)}^{2}\right)+C \varepsilon_{0}^{\prime}\left\|P_{1}\right\|_{W^{1,2}(\Omega)}^{2}+C\left\|P_{3}\right\|_{L^{2}(\Omega)}^{2} .
\end{aligned}
$$

Further, inserting $P_{3}(s)$ as a feasible test function into (4.12), we find with $\varepsilon_{2}(s)>0$

$$
\begin{aligned}
& \frac{1}{2} \frac{\mathrm{d}}{\mathrm{d} s}\left\|P_{3}(s)\right\|_{L^{2}(\Omega)}^{2} \\
& \quad \leqslant \int_{\Omega}\left|\frac{\partial I_{\mathrm{ion}}}{\partial w}\left(\hat{\Phi}_{\mathrm{tr}}, \hat{W}\right)\right|\left|P_{1} P_{3}\right| \mathrm{d} x+\int_{\Omega}\left|\frac{\partial G}{\partial w}\left(\hat{\Phi}_{\mathrm{tr}}, \hat{W}\right)\right|\left|P_{3}\right|^{2} \mathrm{~d} x+\int_{\Omega}\left|\frac{\partial r}{\partial w}\left(\hat{\Phi}_{\mathrm{tr}}, \hat{\Phi}_{e}, \hat{W}\right)\right|\left|P_{3}\right| \mathrm{d} x
\end{aligned}
$$

\footnotetext{
${ }^{13}$ Note that $\varepsilon>0$ is fixed from the Rogers-McCulloch model.
} 


$$
\begin{aligned}
& \leqslant \int_{\Omega}\left|\hat{\Phi}_{\operatorname{tr}} P_{1} P_{3}\right| \mathrm{d} x+\varepsilon\left\|P_{3}\right\|_{L^{2}(\Omega)}^{2}+C\left\|\frac{\partial r}{\partial w}\left(\hat{\Phi}_{\mathrm{tr}}, \hat{\Phi}_{e}, \hat{W}\right)\right\|\left\|_{L^{2}(\Omega)}^{2}+C\right\| P_{3} \|_{L^{2}(\Omega)}^{2} \\
& \leqslant C \varepsilon_{2}(s) \int_{\Omega}\left|\hat{\Phi}_{\operatorname{tr}} P_{1}\right|^{2} \mathrm{~d} x+C\left(1+\frac{1}{\varepsilon_{2}(s)}\right)\left\|P_{3}\right\|_{L^{2}(\Omega)}^{2}+C\left\|\frac{\partial r}{\partial w}\left(\hat{\Phi}_{\mathrm{tr}}, \hat{\Phi}_{e}, \hat{W}\right)\right\| \|_{L^{2}(\Omega)}^{2} \\
& \leqslant C \varepsilon_{2}(s)\left\|\hat{\Phi}_{\operatorname{tr}}\right\|_{L^{4}(\Omega)}^{2} \cdot\left\|P_{1}\right\|_{W^{1,2}(\Omega)}^{2}+C\left(1+\frac{1}{\varepsilon_{2}(s)}\right)\left\|P_{3}\right\|_{L^{2}(\Omega)}^{2}+C\left\|\frac{\partial r}{\partial w}\left(\hat{\Phi}_{\mathrm{tr}}, \hat{\Phi}_{e}, \hat{W}\right)\right\|_{L^{2}(\Omega)} .
\end{aligned}
$$

Choosing now $\varepsilon_{2}(s)=\varepsilon_{2}^{\prime} /\left(1+\left\|\hat{\Phi}_{\mathrm{tr}}(s)\right\|_{L^{4}(\Omega)}^{2}\right)$ with $\varepsilon_{2}^{\prime}>0$, (4.43) may be continued as

$$
\ldots \leqslant C \varepsilon_{2}^{\prime}\left\|P_{1}\right\|_{W^{1,2}(\Omega)}^{2}+C\left(1+\frac{1}{\varepsilon_{2}^{\prime}}\left(1+\left\|\hat{\Phi}_{\mathrm{tr}}(s)\right\|_{L^{4}(\Omega)}^{2}\right)\right)\left\|P_{3}\right\|_{L^{2}(\Omega)}^{2}+C\left\|\frac{\partial r}{\partial w}\left(\hat{\Phi}_{\mathrm{tr}}, \hat{\Phi}_{e}, \hat{W}\right)\right\|_{L^{2}(\Omega)}^{2} .
$$

Combining (4.39) and (4.44), we obtain

$$
\begin{aligned}
\frac{\mathrm{d}}{\mathrm{d} s}( & \left.\left\|P_{1}(s)\right\|_{L^{2}(\Omega)}^{2}+\left\|P_{3}(s)\right\|_{L^{2}(\Omega)}^{2}\right)+2 \beta\left\|P_{1}\right\|_{W^{1,2}(\Omega)}^{2} \\
\leqslant & C\left(\varepsilon_{0}^{\prime}+\varepsilon_{1}^{\prime}+\varepsilon_{2}^{\prime}\right)\left\|P_{1}\right\|_{W^{1,2}(\Omega)}^{2}+C\left(1+\frac{1}{\varepsilon_{1}^{\prime}}\left(1+\left\|\hat{\Phi}_{\operatorname{tr}}(s)\right\|_{L^{4}(\Omega)}^{2}+\|\hat{W}(s)\|_{L^{4}(\Omega)}^{2}\right)\right)\left\|P_{1}\right\|_{L^{2}(\Omega)}^{2} \\
& +C\left(1+\frac{1}{\varepsilon_{2}^{\prime}}\left(1+\left\|\hat{\Phi}_{\mathrm{tr}}(s)\right\|_{L^{4}(\Omega)}^{2}\right)\right)\left\|P_{3}\right\|_{L^{2}(\Omega)}^{2} \\
& +\frac{C}{\varepsilon_{0}^{\prime}}\left(\left\|\frac{\partial r}{\partial \varphi}\left(\hat{\Phi}_{\mathrm{tr}}, \hat{\Phi}_{e}, \hat{W}\right)\right\|_{L^{2}(\Omega)}^{2}+\left\|\frac{\partial r}{\partial \eta}\left(\hat{\Phi}_{\mathrm{tr}}, \hat{\Phi}_{e}, \hat{W}\right)\right\| \|_{L^{2}(\Omega)}^{2}\right)+C\left\|\frac{\partial r}{\partial w}\left(\hat{\Phi}_{\mathrm{tr}}, \hat{\Phi}_{e}, \hat{W}\right)\right\|_{L^{2}(\Omega)} .
\end{aligned}
$$

Now we fix the parameters $\varepsilon_{0}^{\prime}, \varepsilon_{1}^{\prime}, \varepsilon_{2}^{\prime}>0$ in such a way that the terms with $\left\|P_{1}\right\|_{W^{1,2}(\Omega)}$ on both sides of (4.45) will be annihilated, thus arriving at

$$
\begin{gathered}
\frac{\mathrm{d}}{\mathrm{d} s}\left(\left\|P_{1}(s)\right\|_{L^{2}(\Omega)}^{2}+\left\|P_{3}(s)\right\|_{L^{2}(\Omega)}^{2}\right) \leqslant A(s)\left(\left\|P_{1}(s)\right\|_{L^{2}(\Omega)}^{2}+\left\|P_{3}(s)\right\|_{L^{2}(\Omega)}^{2}\right)+B(s) \quad \text { where } \\
\left.A(s)=C\left(1+\left\|\hat{\Phi}_{\mathrm{tr}}(s)\right\|_{L^{4}(\Omega)}^{2}+\|\hat{W}(s)\|_{L^{4}(\Omega)}^{2}\right)\right) ; \\
B(s)=C\left(\left\|\frac{\partial r}{\partial \varphi}\left(\hat{\Phi}_{\mathrm{tr}}, \hat{\Phi}_{e}, \hat{W}\right)\right\|_{L^{2}(\Omega)}^{2}+\left\|\frac{\partial r}{\partial \eta}\left(\hat{\Phi}_{\mathrm{tr}}, \hat{\Phi}_{e}, \hat{W}\right)\right\|_{L^{2}(\Omega)}^{2}+\left\|\frac{\partial r}{\partial w}\left(\hat{\Phi}_{\mathrm{tr}}, \hat{\Phi}_{e}, \hat{W}\right)\right\|_{L^{2}(\Omega)}\right) .
\end{gathered}
$$

Then Gronwall's inequality yields for all $s \in[0, T]$ :

$$
\begin{aligned}
& \left\|P_{1}(s)\right\|_{L^{2}(\Omega)}^{2}+\left\|P_{3}(s)\right\|_{L^{2}(\Omega)}^{2} \leqslant \mathrm{e}^{\int_{0}^{s} A(\sigma) \mathrm{d} \sigma}\left(\left\|P_{1}(0)\right\|_{L^{2}(\Omega)}^{2}+\left\|P_{3}(0)\right\|_{L^{2}(\Omega)}^{2}+\int_{0}^{s} B(\sigma) \mathrm{d} \sigma\right) \\
& \leqslant \mathrm{e}^{C T}\left(\left\|\frac{\partial r}{\partial \varphi}\left(\hat{\Phi}_{\mathrm{tr}}, \hat{\Phi}_{e}, \hat{W}\right)\right\|_{L^{2}\left(\Omega_{T}\right)}^{2}+\left\|\frac{\partial r}{\partial \eta}\left(\hat{\Phi}_{\mathrm{tr}}, \hat{\Phi}_{e}, \hat{W}\right)\right\|_{L^{2}\left(\Omega_{T}\right)}^{2}+\left\|\frac{\partial r}{\partial w}\left(\hat{\Phi}_{\mathrm{tr}}, \hat{\Phi}_{e}, \hat{W}\right)\right\| \|_{L^{2}\left(\Omega_{T}\right)}^{2}\right)
\end{aligned}
$$

since $\hat{\Phi}_{\mathrm{tr}} \in L^{4}\left(\Omega_{T}\right)$ and $\hat{W} \in C^{0}\left[[0, T], L^{4}(\Omega)\right]$. Consequently, we get the estimate

$$
\begin{aligned}
\left\|P_{1}\right\|_{L^{\infty}\left[(0, T), L^{2}(\Omega)\right]}^{2}+\left\|P_{3}\right\|_{L^{\infty}\left[(0, T), L^{2}(\Omega)\right]}^{2} \leqslant & C\left(\left\|\frac{\partial r}{\partial \varphi}\left(\hat{\Phi}_{\mathrm{tr}}, \hat{\Phi}_{e}, \hat{W}\right)\right\| \begin{array}{l}
2 \\
L^{2}\left(\Omega_{T}\right)
\end{array}\right. \\
& \left.+\left\|\frac{\partial r}{\partial \eta}\left(\hat{\Phi}_{\mathrm{tr}}, \hat{\Phi}_{e}, \hat{W}\right)\right\| \begin{array}{l}
2 \\
L^{2}\left(\Omega_{T}\right)
\end{array}+\left\|\frac{\partial r}{\partial w}\left(\hat{\Phi}_{\mathrm{tr}}, \hat{\Phi}_{e}, \hat{W}\right)\right\| \begin{array}{l}
2 \\
L^{2}\left(\Omega_{T}\right)
\end{array}\right) .
\end{aligned}
$$


- Step 3. The estimate for $\left\|P_{1}\right\|_{L^{2}\left[(0, T), W^{1,2}(\Omega)\right]}^{2}$. We return to (4.45). Then $\varepsilon_{0}^{\prime}, \varepsilon_{1}^{\prime}, \varepsilon_{2}^{\prime}>0$ may be alternatively chosen in such a way that $C\left(\varepsilon_{0}^{\prime}+\varepsilon_{1}^{\prime}+\varepsilon_{2}^{\prime}\right)=\beta$ and, consequently,

$$
\begin{gathered}
\frac{\mathrm{d}}{\mathrm{d} s}\left(\left\|P_{1}(s)\right\|_{L^{2}(\Omega)}^{2}+\left\|P_{3}(s)\right\|_{L^{2}(\Omega)}^{2}\right)+\beta\left\|P_{1}(s)\right\|_{W^{1,2}(\Omega)}^{2} \\
\leqslant A(s)\left(\left\|P_{1}(s)\right\|_{L^{2}(\Omega)}^{2}+\left\|P_{3}(s)\right\|_{L^{2}(\Omega)}^{2}\right)+B(s)
\end{gathered}
$$

where $A(s)$ and $B(s)$ are calculated as above. Together with (4.51), we obtain

$$
\begin{gathered}
\frac{\mathrm{d}}{\mathrm{d} s}\left(\left\|P_{1}(s)\right\|_{L^{2}(\Omega)}^{2}+\left\|P_{3}(s)\right\|_{L^{2}(\Omega)}^{2}\right)+\beta\left\|P_{1}(s)\right\|_{W^{1,2}(\Omega)}^{2} \\
\leqslant C A(s)\left(\left\|\frac{\partial r}{\partial \varphi}\left(\hat{\Phi}_{\mathrm{tr}}, \hat{\Phi}_{e}, \hat{W}\right)\right\|_{L^{2}\left(\Omega_{T}\right)}^{2}+\left\|\frac{\partial r}{\partial \eta}\left(\hat{\Phi}_{\mathrm{tr}}, \hat{\Phi}_{e}, \hat{W}\right)\right\|\left\|_{L^{2}\left(\Omega_{T}\right)}^{2}+\right\| \frac{\partial r}{\partial w}\left(\hat{\Phi}_{\mathrm{tr}}, \hat{\Phi}_{e}, \hat{W}\right) \|_{L^{2}\left(\Omega_{T}\right)}^{2}\right)+B(s) .
\end{gathered}
$$

We integrate (4.53) over $[0, T]$ and get, inserting the initial values $P_{1}(0)=\mathfrak{o}, P_{3}(0)=\mathfrak{o}$

$$
\begin{aligned}
& \left\|P_{1}(T)\right\|_{L^{2}(\Omega)}^{2}+\left\|P_{3}(T)\right\|_{L^{2}(\Omega)}^{2}+\beta\left\|P_{1}\right\|_{L^{2}\left[(0, T), W^{1,2}(\Omega)\right]}^{2} \\
& \quad \leqslant C\left(\left\|\frac{\partial r}{\partial \varphi}\left(\hat{\Phi}_{\mathrm{tr}}, \hat{\Phi}_{e}, \hat{W}\right)\right\|_{L^{2}\left(\Omega_{T}\right)}^{2}+\left\|\frac{\partial r}{\partial \eta}\left(\hat{\Phi}_{\mathrm{tr}}, \hat{\Phi}_{e}, \hat{W}\right)\right\|_{L^{2}\left(\Omega_{T}\right)}^{2}+\left\|\frac{\partial r}{\partial w}\left(\hat{\Phi}_{\mathrm{tr}}, \hat{\Phi}_{e}, \hat{W}\right)\right\|_{L^{2}\left(\Omega_{T}\right)}^{2}\right) \Longrightarrow \\
& \left.\left\|P_{1}\right\|_{L^{2}\left[(0, T), W^{1,2}(\Omega)\right.}^{2}\right] \\
& \quad \leqslant C\left(\left\|\frac{\partial r}{\partial \varphi}\left(\hat{\Phi}_{\mathrm{tr}}, \hat{\Phi}_{e}, \hat{W}\right)\right\|_{L^{2}\left(\Omega_{T}\right)}^{2}+\left\|\frac{\partial r}{\partial \eta}\left(\hat{\Phi}_{\mathrm{tr}}, \hat{\Phi}_{e}, \hat{W}\right)\right\|_{L^{2}\left(\Omega_{T}\right)}^{2}+\left\|\frac{\partial r}{\partial w}\left(\hat{\Phi}_{\mathrm{tr}}, \hat{\Phi}_{e}, \hat{W}\right)\right\|_{L^{2}\left(\Omega_{T}\right)}^{2}\right) .
\end{aligned}
$$

- Step 4. The estimate for $\left\|\partial P_{1} / \partial s\right\|_{L^{q}\left[(0, T),\left(W^{1,2}(\Omega)\right)^{*}\right]}^{2}$ with $q=10 / 9<2$. Exploiting the definition of the dual norm, we start with

$$
\begin{aligned}
&\left\|\partial P_{1} / \partial s\right\|_{L^{q}\left[(0, T),\left(W^{1,2}(\Omega)\right)^{*}\right]}^{q}=\int_{0}^{T} \sup _{\|\psi\|_{W^{1,2}(\Omega)}=1}\left|\left\langle\partial P_{1}(s) / \partial s, \psi\right\rangle\right|^{q} \mathrm{~d} s \\
&=\int_{0}^{T} \sup _{\ldots}\left|-A\left(P_{1}, \psi\right)-\int_{\Omega}\left(\frac{\partial I_{\mathrm{ion}}}{\partial \varphi}\left(\hat{\Phi}_{\mathrm{tr}}, \hat{W}\right) P_{1}+\frac{\partial G}{\partial \varphi}\left(\hat{\Phi}_{\mathrm{tr}}, \hat{W}\right) P_{3}\right) \psi \mathrm{d} x+\langle\widetilde{S}(s), \psi\rangle\right|^{q} \mathrm{~d} s \\
& \leqslant C \int_{0}^{T}\left(\underset{\ldots}{\sup }\left|A\left(P_{1}, \psi\right)\right|^{q}+\sup _{\ldots}\left(\int_{\Omega}\left(\left|\hat{\Phi}_{\mathrm{tr}}\right|^{2}+\left|\hat{\Phi}_{\mathrm{tr}}\right|+|\hat{W}|+1\right)\left|P_{1}\right||\psi| \mathrm{d} x\right)^{q}\right. \\
&\left.+\sup _{\ldots} \varepsilon^{q} \kappa^{q}\left(\int_{\Omega}\left|P_{3}\right||\psi| \mathrm{d} x\right)^{q}+\sup _{\ldots}|\langle\widetilde{S}(s), \psi\rangle|^{q}\right) \mathrm{d} s .
\end{aligned}
$$

The four terms on the right-hand side of (4.58) will be estimated separately. For the first term, we get with [12], page 6 , Theorem $2.4,2)$, and $(4.55)$

$$
\begin{aligned}
& \int_{0}^{T} \sup _{\ldots}\left|A\left(P_{1}, \psi\right)\right|^{q} \mathrm{~d} s \leqslant \int_{0}^{T} \sup _{\ldots} \gamma^{q}\left\|P_{1}(s)\right\|_{W^{1,2}(\Omega)}^{q}\|\psi\|_{W^{1,2}(\Omega)}^{q} \mathrm{~d} s \\
& \leqslant \gamma^{q}\left\|P_{1}\right\|_{L^{q}\left[(0, T), W^{1,2}(\Omega)\right]}^{q} \leqslant C\left\|P_{1}\right\|_{L^{2}\left[(0, T), W^{1,2}(\Omega)\right]}^{q} \\
& \leqslant C\left(\left\|\frac{\partial r}{\partial \varphi}\left(\hat{\Phi}_{\mathrm{tr}}, \hat{\Phi}_{e}, \hat{W}\right)\right\|_{L^{2}\left(\Omega_{T}\right)}^{2}+\left\|\frac{\partial r}{\partial \eta}\left(\hat{\Phi}_{\mathrm{tr}}, \hat{\Phi}_{e}, \hat{W}\right)\right\|_{L^{2}\left(\Omega_{T}\right)}^{2}+\left\|\frac{\partial r}{\partial w}\left(\hat{\Phi}_{\mathrm{tr}}, \hat{\Phi}_{e}, \hat{W}\right)\right\|_{L^{2}\left(\Omega_{T}\right)}^{2}\right)^{q / 2} .
\end{aligned}
$$

For the second term, we obtain

$$
\int_{0}^{T} \sup \left(\int_{\Omega}\left(\left|\hat{\Phi}_{\mathrm{tr}}\right|^{2}+\left|\hat{\Phi}_{\mathrm{tr}}\right|+|\hat{W}|+1\right)\left|P_{1}\right||\psi| \mathrm{d} x\right)^{q} \mathrm{~d} s
$$


1092

K. KUNISCH AND M. WAGNER

$$
\begin{aligned}
\leqslant & C\left(\sup _{\ldots} \int_{0}^{T} \int_{\Omega}\left|\hat{\Phi}_{\mathrm{tr}}\right|^{2 q}\left|P_{1}\right|^{q}|\psi|^{q} \mathrm{~d} x \mathrm{~d} s+\sup _{\ldots} \int_{0}^{T} \int_{\Omega}\left|\hat{\Phi}_{\mathrm{tr}}\right|^{q}\left|P_{1}\right|^{q}|\psi|^{q} \mathrm{~d} x \mathrm{~d} s\right. \\
& \left.+\sup _{\ldots} \int_{0}^{T} \int_{\Omega}|\hat{W}|^{q}\left|P_{1}\right|^{q}|\psi|^{q} \mathrm{~d} x \mathrm{~d} s+\sup _{\ldots} \int_{0}^{T} \int_{\Omega}\left|P_{1}\right|^{q}|\psi|^{q} \mathrm{~d} x \mathrm{~d} s\right)=J_{1}+J_{2}+J_{3}+J_{4} .
\end{aligned}
$$

We start with the estimation of $J_{1}$, thus getting

$$
\begin{aligned}
J_{1}= & \sup _{\ldots} \int_{0}^{T} \int_{\Omega}\left|\hat{\Phi}_{\mathrm{tr}}\right|^{20 / 9}\left|P_{1}\right|^{10 / 9}|\psi|^{10 / 9} \mathrm{~d} x \mathrm{~d} s \\
& =\sup _{\ldots} \int_{0}^{T}\left(\int_{\Omega}\left|\hat{\Phi}_{\mathrm{tr}}\right|^{40 / 11} \mathrm{~d} x\right)^{33 / 54}\left(\int_{\Omega}\left|P_{1}\right|^{60 / 11} \mathrm{~d} x\right)^{11 / 54}\left(\int_{\Omega}|\psi|^{6} \mathrm{~d} x\right)^{10 / 54} \mathrm{~d} s \\
& =\sup \int_{0}^{T}\left\|\hat{\Phi}_{\mathrm{tr}}\right\|_{L^{40 / 11}(\Omega)}^{20 / 9} \cdot\left\|P_{1}\right\|_{L^{60 / 11}(\Omega)}^{10 / 9} \cdot\|\psi\|_{L^{6}(\Omega)}^{10 / 9} \mathrm{~d} s \\
\leqslant & \sup _{\quad} C \int_{0}^{T}\left\|\hat{\Phi}_{\mathrm{tr}}\right\|_{L^{40 / 11}(\Omega)}^{20 / 9} \cdot\left\|P_{1}\right\|_{W^{1,2}(\Omega)}^{10 / 9} \cdot\|\psi\|_{W^{1,2}(\Omega)}^{10 / 9} \mathrm{~d} s=C \int_{0}^{T}\left\|\hat{\Phi}_{\mathrm{tr}}\right\|_{L^{40 / 11}(\Omega)}^{20 / 9} \cdot\left\|P_{1}\right\|_{W^{1,2}(\Omega)}^{10 / 9} \mathrm{~d} s \\
& \leqslant C\left(\int_{0}^{T}\left\|\hat{\Phi}_{\operatorname{tr}}(s)\right\|_{L^{40 / 11}(\Omega)}^{5} \mathrm{~d} s\right)^{8 / 18}\left(\int_{0}^{T}\left\|P_{1}(s)\right\|_{W^{1,2}(\Omega)}^{2} \mathrm{~d} s\right)^{10 / 18} \\
& \leqslant C \cdot\left\|\hat{\Phi}_{\mathrm{tr}}\right\|_{L^{5}\left[(0, T), L^{40 / 11}(\Omega)\right.}^{20 / 9} \cdot\left\|P_{1}\right\|_{L^{2}\left[(0, T), W^{1,2}(\Omega)\right]}^{10 / 9} \cdot
\end{aligned}
$$

Since $\hat{\Phi}_{\operatorname{tr}} \in L^{p^{\prime}}\left[(0, T), L^{p^{\prime \prime}}(\Omega)\right]$ for all $1<p^{\prime}<\infty, 4 \leqslant p^{\prime \prime}<6$, we get a bound analogous to (4.61). Continuing with $J_{2}$, we find in completely analogous manner

$$
\begin{aligned}
& J_{2}=\sup _{\ldots} \int_{0}^{T} \int_{\Omega}\left|\hat{\Phi}_{\mathrm{tr}}\right|^{10 / 9}\left|P_{1}\right|^{10 / 9}|\psi|^{10 / 9} \mathrm{~d} x \mathrm{~d} s \\
& \leqslant \sup _{\ldots} C \int_{0}^{T}\left\|\hat{\Phi}_{\operatorname{tr}}\right\|_{L^{20 / 11}(\Omega)}^{10 / 9} \cdot\left\|P_{1}\right\|_{W^{1,2}(\Omega)}^{10 / 9} \cdot\|\psi\|_{W^{1,2}(\Omega)}^{10 / 9} \mathrm{~d} s=C \int_{0}^{T}\left\|\hat{\Phi}_{\mathrm{tr}}\right\|_{L^{20 / 11}(\Omega)}^{10 / 9} \cdot\left\|P_{1}\right\|_{W^{1,2}(\Omega)}^{10 / 9} \mathrm{~d} s \\
& \leqslant C\left(\int_{0}^{T}\left\|\hat{\Phi}_{\operatorname{tr}}(s)\right\|_{L^{20 / 11}(\Omega)}^{10 / 4} \mathrm{~d} s\right)^{8 / 18}\left(\int_{0}^{T}\left\|P_{1}(s)\right\|_{W^{1,2}(\Omega)}^{2} \mathrm{~d} s\right)^{10 / 18} \\
& \leqslant C \cdot\left\|\hat{\Phi}_{\operatorname{tr}}\right\|_{L^{10 / 4}\left[(0, T), L^{20 / 11}(\Omega)\right]}^{10 / 9} \cdot\left\|P_{1}\right\|_{L^{2}\left[(0, T), W^{1,2}(\Omega)\right]}^{10 / 9} \cdot
\end{aligned}
$$

Noticing that, in the case of the Rogers-McCulloch model, $\hat{W}$ belongs even to $C^{0}\left[[0, T], L^{4}(\Omega)\right]$, we may estimate $J_{3}$ in the same way:

$$
\begin{aligned}
& J_{3}=\underset{\ldots}{\sup } \int_{0}^{T} \int_{\Omega}|\hat{W}|^{10 / 9}\left|P_{1}\right|^{10 / 9}|\psi|^{10 / 9} \mathrm{~d} x \mathrm{~d} s \\
& \leqslant \sup _{\ldots} C \int_{0}^{T}\|\hat{W}\|_{L^{20 / 11}(\Omega)}^{10 / 9} \cdot\left\|P_{1}\right\|_{W^{1,2}(\Omega)}^{10 / 9} \cdot\|\psi\|_{W^{1,2}(\Omega)}^{10 / 9} \mathrm{~d} s=C \int_{0}^{T}\|\hat{W}\|_{L^{20 / 11}(\Omega)}^{10 / 9} \cdot\left\|P_{1}\right\|_{W^{1,2}(\Omega)}^{10 / 9} \mathrm{~d} s \\
& \leqslant C\left(\int_{0}^{T}\|\hat{W}(s)\|_{L^{20 / 11}(\Omega)}^{10 / 4} \mathrm{~d} s\right)^{8 / 18}\left(\int_{0}^{T}\left\|P_{1}(s)\right\|_{W^{1,2}(\Omega)}^{2} \mathrm{~d} s\right)^{10 / 18} \\
& \left.\leqslant C \cdot\|\hat{W}\|_{L^{10 / 4}\left[(0, T), L^{20 / 11}(\Omega)\right]}^{10 / 9} \cdot\left\|P_{1}\right\|_{L^{2}\left[(0, T), W^{1,2}(\Omega)\right]}^{10 / 9}\right]
\end{aligned}
$$


OPTIMAL CONTROL OF THE BIDOMAIN SYSTEM (III): OPTIMALITY CONDITIONS

$$
\leqslant C \cdot\|\hat{W}\|_{L^{\infty}\left[(0, T), L^{4}(\Omega)\right]}^{10 / 9} \cdot\left\|P_{1}\right\|_{L^{2}\left[(0, T), W^{1,2}(\Omega)\right]}^{10 / 9} .
$$

Defining the function $S(x, s) \equiv 1$, the estimation of $J_{4}$ yields

$$
\begin{gathered}
J_{4}=\sup _{\ldots} \int_{0}^{T} \int_{\Omega}|S|^{10 / 9}\left|P_{1}\right|^{10 / 9}|\psi|^{10 / 9} \mathrm{~d} x \mathrm{~d} s \\
\leqslant C \cdot\|S\|_{L^{10 / 4}\left[(0, T), L^{20 / 11}(\Omega)\right]}^{10 / 9} \cdot\left\|P_{1}\right\|_{L^{2}\left[(0, T), W^{1,2}(\Omega)\right]}^{10 / 9}
\end{gathered}
$$

Summing up, we get from (4.63), (4.69), (4.73), (4.78) and (4.79):

$$
\begin{aligned}
& \int_{0}^{T} \sup _{\ldots}\left(\int_{\Omega}\left(\left|\hat{\Phi}_{\mathrm{tr}}\right|^{2}+\left|\hat{\Phi}_{\mathrm{tr}}\right|+|\hat{W}|+1\right)\left|P_{1}\right||\psi| \mathrm{d} x\right)^{q} \mathrm{~d} s \\
& \quad \leqslant C\left(\left\|\frac{\partial r}{\partial \varphi}\left(\hat{\Phi}_{\mathrm{tr}}, \hat{\Phi}_{e}, \hat{W}\right)\right\|_{L^{2}\left(\Omega_{T}\right)}^{2}+\left.\left\|\frac{\partial r}{\partial \eta}\left(\hat{\Phi}_{\mathrm{tr}}, \hat{\Phi}_{e}, \hat{W}\right)\right\|\right|_{L^{2}\left(\Omega_{T}\right)} ^{2}+\left\|\frac{\partial r}{\partial w}\left(\hat{\Phi}_{\mathrm{tr}}, \hat{\Phi}_{e}, \hat{W}\right)\right\| \|_{L^{2}\left(\Omega_{T}\right)}\right)^{q / 2} .
\end{aligned}
$$

The third term at the right-hand side of (4.58) will be estimated through

$$
\begin{aligned}
& \int_{0}^{T} \sup _{\ldots} \varepsilon^{q} \kappa^{q}\left(\int_{\Omega}\left|P_{3}\right||\psi| \mathrm{d} x\right)^{q} \mathrm{~d} s \leqslant \sup _{\ldots} C \int_{0}^{T} \int_{\Omega}\left|P_{3}\right|^{10 / 9}|\psi|^{10 / 9} \mathrm{~d} x \mathrm{~d} s \\
& \leqslant \sup _{\ldots} C \int_{0}^{T}\left(\int_{\Omega}\left|P_{3}\right|^{2} \mathrm{~d} x\right)^{10 / 18}\left(\int_{\Omega}|\psi|^{10 / 4} \mathrm{~d} x\right)^{8 / 18} \mathrm{~d} s=\sup _{\ldots} C \int_{0}^{T}\left\|P_{3}\right\|_{L^{2}(\Omega)}^{10 / 9} \cdot\|\psi\|_{L^{10 / 4}(\Omega)}^{10 / 9} \\
& \leqslant \sup _{\ldots} C \int_{0}^{T}\left\|P_{3}\right\|_{L^{2}(\Omega)}^{10 / 9} \cdot\|\psi\|_{W^{1,2}(\Omega)}^{10 / 9}=C\left\|P_{3}\right\|_{L^{q}\left[(0, T), L^{2}(\Omega)\right.}^{q} \leqslant C\left\|P_{3}\right\|_{L^{\infty}\left[(0, T), L^{2}(\Omega)\right]}^{q} \leqslant \\
& \leqslant C\left(\left\|\frac{\partial r}{\partial \varphi}\left(\hat{\Phi}_{\mathrm{tr}}, \hat{\Phi}_{e}, \hat{W}\right)\right\|_{L^{2}\left(\Omega_{T}\right)}^{2}+\left\|\frac{\partial r}{\partial \eta}\left(\hat{\Phi}_{\mathrm{tr}}, \hat{\Phi}_{e}, \hat{W}\right)\right\|_{L^{2}\left(\Omega_{T}\right)}^{2}+\left\|\frac{\partial r}{\partial w}\left(\hat{\Phi}_{\mathrm{tr}}, \hat{\Phi}_{e}, \hat{W}\right)\right\|{ }_{L^{2}\left(\Omega_{T}\right)}^{2}\right)^{q / 2} .
\end{aligned}
$$

Finally, Lemma 4.3 implies for the fourth term at the right-hand side of (4.58):

$$
\begin{aligned}
& \int_{0}^{T} \sup _{\ldots}|\langle\widetilde{S}(s), \psi\rangle|^{q} \mathrm{~d} s \leqslant C\left(1+\int_{0}^{T}\left(\left\|\frac{\partial r}{\partial \varphi}\left(\hat{\Phi}_{\mathrm{tr}}, \hat{\Phi}_{e}, \hat{W}\right)\right\|_{L^{2}(\Omega)}^{2}+\left\|\frac{\partial r}{\partial \eta}\left(\hat{\Phi}_{\mathrm{tr}}, \hat{\Phi}_{e}, \hat{W}\right)\right\|_{L^{2}(\Omega)}^{2}\right)^{q} \mathrm{~d} s\right)^{2} \\
& \leqslant C\left(1+\int_{0}^{T}\left(\left\|\frac{\partial r}{\partial \varphi}\left(\hat{\Phi}_{\mathrm{tr}}, \hat{\Phi}_{e}, \hat{W}\right)\right\|_{L^{2}(\Omega)}^{2 q}+\left\|\frac{\partial r}{\partial \eta}\left(\hat{\Phi}_{\mathrm{tr}}, \hat{\Phi}_{e}, \hat{W}\right)\right\|_{L^{2}(\Omega)}^{2 q}\right) \mathrm{d} s\right) \\
& \leqslant C\left(1+\left\|\frac{\partial r}{\partial \varphi}\left(\hat{\Phi}_{\mathrm{tr}}, \hat{\Phi}_{e}, \hat{W}\right)\right\|_{L^{20 / 9}\left[(0, T), L^{2}(\Omega)\right]}^{20 / 9}+\left\|\frac{\partial r}{\partial \eta}\left(\hat{\Phi}_{\mathrm{tr}}, \hat{\Phi}_{e}, \hat{W}\right)\right\|_{L^{20 / 9}\left[(0, T), L^{2}(\Omega)\right]}^{20 / 9}\right) .
\end{aligned}
$$

Together with (4.61), (4.80), (4.84) and (4.87), (4.58) yields the claimed estimate

$$
\begin{aligned}
& \left\|\partial P_{1} / \partial s\right\|_{L^{q}\left[(0, T),\left(W^{1,2}(\Omega)\right)^{*}\right]}^{q} \\
& \leqslant C\left(\left\|\frac{\partial r}{\partial \varphi}\left(\hat{\Phi}_{\mathrm{tr}}, \hat{\Phi}_{e}, \hat{W}\right)\right\|_{L^{2}\left(\Omega_{T}\right)}^{2}+\left\|\frac{\partial r}{\partial \eta}\left(\hat{\Phi}_{\mathrm{tr}}, \hat{\Phi}_{e}, \hat{W}\right)\right\|_{L_{\left(\Omega_{T}\right)}^{2}}^{2}\left\|\frac{\partial r}{\partial w}\left(\hat{\Phi}_{\mathrm{tr}}, \hat{\Phi}_{e}, \hat{W}\right)\right\|_{L^{2}\left(\Omega_{T}\right)}^{2}\right)^{q / 2} \\
& +C\left(1+\left\|\frac{\partial r}{\partial \varphi}\left(\hat{\Phi}_{\mathrm{tr}}, \hat{\Phi}_{e}, \hat{W}\right)\right\|_{L^{20 / 9}\left[(0, T), L^{2}(\Omega)\right]}^{20 / 9}+\left\|\frac{\partial r}{\partial \eta}\left(\hat{\Phi}_{\mathrm{tr}}, \hat{\Phi}_{e}, \hat{W}\right)\right\|_{L^{20 / 9}\left[(0, T), L^{2}(\Omega)\right]}^{20 / 9}\right) .
\end{aligned}
$$


- Step 5. The estimate for $\left\|\partial P_{3} / \partial s\right\|_{L^{q}\left[(0, T),\left(L^{2}(\Omega)\right)^{*}\right]}^{q}$. We start again by using the dual norm

$$
\begin{aligned}
& \left\|\partial P_{3} / \partial s\right\|_{L^{q}\left[(0, T),\left(L^{2}(\Omega)\right)^{*}\right]}^{q}=\int_{0}^{T} \sup _{\|\psi\|_{L^{2}(\Omega)}=1}\left|\left\langle\partial P_{3}(s) / \partial s, \psi\right\rangle\right|^{q} \mathrm{~d} s \\
& =\int_{0}^{T} \sup _{\ldots}\left|\int_{\Omega}\left(-\hat{\Phi}_{\mathrm{tr}} P_{1}-\varepsilon P_{3}-\frac{\partial r}{\partial w}\left(\hat{\Phi}_{\mathrm{tr}}, \hat{\Phi}_{e}, \hat{W}\right)\right) \psi \mathrm{d} x\right|^{q} \mathrm{~d} s \\
& \leqslant C \int_{0}^{T} \sup _{\ldots}\left(\left(\int_{\Omega}\left|\hat{\Phi}_{\mathrm{tr}}\right|\left|P_{1}\right||\psi| \mathrm{d} x\right)^{q}+\left(\varepsilon \int_{\Omega}\left|P_{3}\right||\psi| \mathrm{d} x\right)^{q}+\left(\int_{\Omega}\left|\frac{\partial r}{\partial w}\left(\hat{\Phi}_{\mathrm{tr}}, \hat{\Phi}_{e}, \hat{W}\right)\right||\psi| \mathrm{d} x\right)^{q}\right) \mathrm{d} s \\
& \leqslant C\left(\sup _{\ldots} \int_{0}^{T} \int_{\Omega}\left|\hat{\Phi}_{\mathrm{tr}}\right|^{q}\left|P_{1}\right|^{q}|\psi|^{q} \mathrm{~d} x \mathrm{~d} s+\sup _{\ldots} \int_{0}^{T}\left(\int_{\Omega}\left|P_{3}\right||\psi| \mathrm{d} x\right)^{q} \mathrm{~d} s\right. \\
& \left.\quad+\sup _{\ldots} \int_{0}^{T}\left(\int_{\Omega}\left|\frac{\partial r}{\partial w}\left(\hat{\Phi}_{\mathrm{tr}}, \hat{\Phi}_{e}, \hat{W}\right)\right||\psi| \mathrm{d} x\right)^{q} \mathrm{~d} s\right)=J_{5}+J_{6}+J_{7} .
\end{aligned}
$$

The three terms on the right-hand side of (4.91) will be estimated separately. For the first term, we get

$$
\begin{aligned}
J_{5}= & \sup _{\cdots} C \int_{0}^{T} \int_{\Omega}\left|\hat{\Phi}_{\mathrm{tr}}\right|^{10 / 9}\left|P_{1}\right|^{10 / 9}|\psi|^{10 / 9} \mathrm{~d} x \mathrm{~d} s \\
& \leqslant \sup _{\cdots} C\left(\int_{0}^{T} \int_{\Omega}\left|\hat{\Phi}_{\mathrm{tr}}\right|^{5} \mathrm{~d} x \mathrm{~d} s\right)^{2 / 9}\left(\int_{0}^{T} \int_{\Omega}\left|P_{1}\right|^{5} \mathrm{~d} x \mathrm{~d} s\right)^{2 / 9}\left(\int_{0}^{T} \int_{\Omega}|\psi|^{2} \mathrm{~d} x \mathrm{~d} s\right)^{5 / 9} \\
& =\sup _{\|\psi(s)\|_{L^{2}(\Omega)}=1} C \cdot\left\|\hat{\Phi}_{\mathrm{tr}}\right\|_{L^{5}\left(\Omega_{T}\right)}^{10 / 9} \cdot\left\|P_{1}\right\|_{L^{5}\left(\Omega_{T}\right)}^{10 / 9} \cdot\left(\int_{0}^{T}\|\psi(s)\|_{L^{2}(\Omega)}^{2} \mathrm{~d} x \mathrm{~d} s\right)^{5 / 9} \\
& \leqslant C \cdot\left\|\hat{\Phi}_{\mathrm{tr}}\right\|_{L^{5}\left(\Omega_{T}\right)}^{10 / 9} \cdot\left(\left\|P_{1}\right\|_{L^{2}\left[(0, T), W^{1,2}(\Omega)\right]}^{10 / 9}+\left\|\partial P_{1} / \partial s\right\|_{L^{10 / 9}\left[(0, T),\left(W^{1,2}(\Omega)\right)^{*}\right]}^{10 / 9}\right) \cdot T^{5 / 9}
\end{aligned}
$$

by application of the Aubin-Dubinskij lemma to $P_{1}$. Since $\hat{\Phi}_{\mathrm{tr}} \in L^{5}\left(\Omega_{T}\right)$, we may use (4.55) and (4.88) in order to conclude that

$$
\begin{aligned}
J_{5} \leqslant C & \left(\left\|\frac{\partial r}{\partial \varphi}\left(\hat{\Phi}_{\mathrm{tr}}, \hat{\Phi}_{e}, \hat{W}\right)\right\|_{L^{2}\left(\Omega_{T}\right)}^{2}+\left\|\frac{\partial r}{\partial \eta}\left(\hat{\Phi}_{\mathrm{tr}}, \hat{\Phi}_{e}, \hat{W}\right)\right\|_{L^{2}\left(\Omega_{T}\right)}^{2}+\left\|\frac{\partial r}{\partial w}\left(\hat{\Phi}_{\mathrm{tr}}, \hat{\Phi}_{e}, \hat{W}\right)\right\|_{L^{2}\left(\Omega_{T}\right)}\right)^{q / 2} \\
& +C\left(1+\left\|\frac{\partial r}{\partial \varphi}\left(\hat{\Phi}_{\mathrm{tr}}, \hat{\Phi}_{e}, \hat{W}\right)\right\|_{L^{20 / 9}\left[(0, T), L^{2}(\Omega)\right]}^{20 / 9}+\left\|\frac{\partial r}{\partial \eta}\left(\hat{\Phi}_{\mathrm{tr}}, \hat{\Phi}_{e}, \hat{W}\right)\right\|_{L^{20 / 9}\left[(0, T), L^{2}(\Omega)\right]}^{20 / 9}\right) .
\end{aligned}
$$

For the second term, we find

$$
\begin{aligned}
J_{6}= & \sup _{\ldots} C \int_{0}^{T}\left(\int_{\Omega}\left|P_{3}\right||\psi| \mathrm{d} x\right)^{q} \mathrm{~d} s \leqslant \sup _{\ldots} C \int_{0}^{T}\left(\left\|P_{3}(s)\right\|_{L^{2}(\Omega)}^{2}+\|\psi(s)\|_{L^{2}(\Omega)}^{2}\right)^{10 / 9} \mathrm{~d} s \\
& \leqslant C\left(1+\left\|P_{3}\right\|_{L^{\infty}\left[(0, T), L^{2}(\Omega)\right]}^{20 / 9}\right) \\
& \leqslant C\left(1+\left\|\frac{\partial r}{\partial \varphi}\left(\hat{\Phi}_{\mathrm{tr}}, \hat{\Phi}_{e}, \hat{W}\right)\right\|_{L^{2}\left(\Omega_{T}\right)}^{2}+\left\|\frac{\partial r}{\partial \eta}\left(\hat{\Phi}_{\mathrm{tr}}, \hat{\Phi}_{e}, \hat{W}\right)\right\|_{L^{2}\left(\Omega_{T}\right)}^{2}+\left\|\frac{\partial r}{\partial w}\left(\hat{\Phi}_{\mathrm{tr}}, \hat{\Phi}_{e}, \hat{W}\right)\right\|_{L^{2}\left(\Omega_{T}\right)}^{2}\right)^{q}
\end{aligned}
$$


by (4.51). For the third term, we get

$$
\begin{aligned}
J_{7}= & \sup _{\ldots} \int_{0}^{T}\left(\int_{\Omega}\left|\frac{\partial r}{\partial w}\left(\hat{\Phi}_{\mathrm{tr}}, \hat{\Phi}_{e}, \hat{W}\right)\right||\psi| \mathrm{d} x\right)^{q} \mathrm{~d} s \leqslant \sup _{\ldots} \int_{0}^{T}\left(\left\|\frac{\partial r}{\partial w}\left(\hat{\Phi}_{\mathrm{tr}}, \hat{\Phi}_{e}, \hat{W}\right)\right\|_{L^{2}(\Omega)}^{2}\right. \\
& \left.+\|\psi(s)\|_{L^{2}(\Omega)}^{2}\right)^{q} \mathrm{~d} s \leqslant C\left(1+\left\|\frac{\partial r}{\partial w}\left(\hat{\Phi}_{\mathrm{tr}}, \hat{\Phi}_{e}, \hat{W}\right)\right\|_{L^{2 q}\left[(0, T), L^{2}(\Omega)\right]}^{20 / 9}\right) .
\end{aligned}
$$

Combining now (4.91) with (4.96), (4.99) and (4.101), we arrive at the claimed estimate

$$
\begin{aligned}
&\left.\left.\left\|\partial P_{3} / \partial s\right\|_{L^{q}\left[(0, T),\left(L^{2}(\Omega)\right.\right.}^{q}\right)^{*}\right] \\
& \leqslant C\left(\left\|\frac{\partial r}{\partial \varphi}\left(\hat{\Phi}_{\mathrm{tr}}, \hat{\Phi}_{e}, \hat{W}\right)\right\|_{L^{2}\left(\Omega_{T}\right)}^{2}+\left\|\frac{\partial r}{\partial \eta}\left(\hat{\Phi}_{\mathrm{tr}}, \hat{\Phi}_{e}, \hat{W}\right)\right\|_{L^{2}\left(\Omega_{T}\right)}^{2}+\left\|\frac{\partial r}{\partial w}\left(\hat{\Phi}_{\mathrm{tr}}, \hat{\Phi}_{e}, \hat{W}\right)\right\|_{L^{2}\left(\Omega_{T}\right)}\right)^{q / 2} \\
&+C\left(1+\left\|\frac{\partial r}{\partial \varphi}\left(\hat{\Phi}_{\mathrm{tr}}, \hat{\Phi}_{e}, \hat{W}\right)\right\|_{L^{20 / 9}\left[(0, T), L^{2}(\Omega)\right]}^{20 / 9}+\left\|\frac{\partial r}{\partial \eta}\left(\hat{\Phi}_{\mathrm{tr}}, \hat{\Phi}_{e}, \hat{W}\right)\right\|_{L^{20 / 9}\left[(0, T), L^{2}(\Omega)\right]}^{20 / 9}\right) .
\end{aligned}
$$

- Step 6. The estimate for $\left\|P_{2}\right\|_{L^{2}\left[(0, T), W^{1,2}(\Omega)\right]}^{2}$. Inserting $P_{2}(s) \in W^{1,2}(\Omega)$ with $\int_{\Omega} P_{2}(x, s) \mathrm{d} x=0$ as a feasible test function into (4.9), the uniform ellipticity of $M_{i}, M_{e}$ and the Poincaré inequality imply

$$
\begin{aligned}
& \left\|P_{2}(s)\right\|_{W^{1,2}(\Omega)}^{2} \leqslant C \int_{\Omega} \nabla P_{2}^{\mathrm{T}}\left(M_{i}+M_{e}\right) \nabla P_{2} \mathrm{~d} x \\
& \leqslant C\left|\int_{\Omega} \nabla P_{2}^{\mathrm{T}} M_{i} \nabla P_{1} \mathrm{~d} x+\int_{\Omega} \frac{\partial r}{\partial \eta}\left(\hat{\Phi}_{\mathrm{tr}}, \hat{\Phi}_{e}, \hat{W}\right) P_{2} \mathrm{~d} x\right| \\
& \leqslant C\left(\left\|P_{1}\right\|_{W^{1,2}(\Omega)} \cdot\left\|P_{2}\right\|_{W^{1,2}(\Omega)}+\int_{\Omega}\left|\frac{\partial r}{\partial \eta}\left(\hat{\Phi}_{\mathrm{tr}}, \hat{\Phi}_{e}, \hat{W}\right)\right|\left|P_{2}\right| \mathrm{d} x\right)
\end{aligned}
$$

since, by Assumption 2.1,2), the entries of $M_{i}$ are essentially bounded. Consequently, applying the generalized Cauchy inequality twice, we get

$$
\begin{aligned}
\left\|P_{2}(s)\right\|_{W^{1,2}(\Omega)}^{2} \leqslant C\left(\frac{1}{\varepsilon_{3}^{\prime}}\left\|P_{1}\right\|_{W^{1,2}(\Omega)}^{2}+\right. & \varepsilon_{3}^{\prime}\left\|P_{2}\right\|_{W^{1,2}(\Omega)}^{2} \\
& \left.+\frac{1}{\varepsilon_{4}^{\prime}}\left\|\frac{\partial r}{\partial \eta}\left(\hat{\Phi}_{\mathrm{tr}}, \hat{\Phi}_{e}, \hat{W}\right)\right\|_{L^{2}(\Omega)}^{2}+\varepsilon_{4}^{\prime}\left\|P_{2}\right\|_{W^{1,2}(\Omega)}^{2}\right)
\end{aligned}
$$

for arbitrary $\varepsilon_{3}^{\prime}, \varepsilon_{4}^{\prime}>0$. Choosing $\left(\varepsilon_{3}^{\prime}+\varepsilon_{4}^{\prime}\right)=1 /(2 C)$, we arrive at

$$
\begin{aligned}
& \frac{1}{2}\left\|P_{2}(s)\right\|_{W^{1,2}(\Omega)}^{2} \leqslant C\left(\left\|P_{1}\right\|_{W^{1,2}(\Omega)}^{2}+\left\|\frac{\partial r}{\partial \eta}\left(\hat{\Phi}_{\mathrm{tr}}, \hat{\Phi}_{e}, \hat{W}\right)\right\|_{L^{2}(\Omega)}^{2}\right) \Longrightarrow \\
& \left\|P_{2}\right\|_{L^{2}\left[(0, T), W^{1,2}(\Omega)\right]}^{2} \leqslant C\left(\left\|P_{1}\right\|_{L^{2}\left[(0, T), W^{1,2}(\Omega)\right]}^{2}+\left\|\frac{\partial r}{\partial \eta}\left(\hat{\Phi}_{\mathrm{tr}}, \hat{\Phi}_{e}, \hat{W}\right)\right\|_{L^{2}\left(\Omega_{T}\right)}^{2}\right)
\end{aligned}
$$

where the right-hand side is bounded by (4.55).

- Step 7. Conclusion of the proof. The fact that $P_{1}$ belongs even to $C^{0}\left[[0, T], L^{2}(\Omega)\right]$ can be confirmed analogously to [4], page 478, Section 5.3. As a consequence of the imbedding theorem [7], page 286, Theorem 2, $P_{3} \in C^{0}\left[[0, T], L^{2}(\Omega)\right]$ holds true as well. Consequently, the norms on the left-hand side of (4.51) can be replaced by $C^{0}\left[[0, T], L^{2}(\Omega)\right]$-norms, and the proof is complete. 
Proof of Theorem 4.2.

- Step 1. Approximate solutions for the reduced adjoint system. By [4], page 464, Theorem 6, the bidomain bilinear form $A(\cdot, \cdot)$ gives rise to an orthonormal basis of eigenfunctions $\left\{\psi_{i}\right\}$ within the space $W^{1,2}(\Omega)$, which are related to eigenvalues $0=\lambda_{0}<\lambda_{1} \leqslant \lambda_{2} \leqslant \ldots$ For $N \in \mathbb{N}_{0}$, let us define the subspaces

$$
\mathrm{X}^{N}(\Omega)=\left\{\psi=\sum_{i=0}^{N} c_{i} \psi_{i} \mid c_{0}, \ldots, c_{N} \in \mathbb{R}\right\} \subset W^{1,2}(\Omega),
$$

and the functions $P_{1}^{N}, P_{3}^{N}: \Omega \times[0, T] \rightarrow \mathrm{X}^{N}$ according to

$$
P_{1}^{N}(x, s)=\sum_{i=0}^{N} p_{i, N}(s) \psi_{i}(x) ; \quad P_{3}^{N}(x, s)=\sum_{i=0}^{N} q_{i, N}(s) \psi_{i}(x)
$$

where $p_{i, N}, q_{i, N}:[0, T] \rightarrow \mathbb{R}$ are solutions of the initial value problem

$$
\begin{aligned}
& \frac{\mathrm{d} p_{j, N}(s)}{\mathrm{d} s}+\lambda_{j} p_{j, N}(s)+\sum_{i=0}^{N} p_{i, N}(s) \cdot \int_{\Omega} \frac{\partial I_{\mathrm{ion}}}{\partial \varphi}\left(\hat{\Phi}_{\mathrm{tr}}, \hat{W}\right) \psi_{i}(x) \psi_{j}(x) \mathrm{d} x \\
& \quad+\sum_{i=0}^{N} q_{i, N}(s) \cdot \int_{\Omega} \frac{\partial G}{\partial \varphi}\left(\hat{\Phi}_{\mathrm{tr}}, \hat{W}\right) \psi_{i}(x) \psi_{j}(x) \mathrm{d} x=\left\langle\widetilde{S}(s), \psi_{j}\right\rangle, \quad 0 \leqslant j \leqslant N ; \\
& \frac{\mathrm{d} q_{j, N}(s)}{\mathrm{d} s}+\sum_{i=0}^{N} p_{i, N}(s) \cdot \int_{\Omega} \frac{\partial I_{\mathrm{ion}}}{\partial w}\left(\hat{\Phi}_{\mathrm{tr}}, \hat{W}\right) \psi_{i}(x) \psi_{j}(x) \mathrm{d} x \\
& +\sum_{i=0}^{N} q_{i, N}(s) \cdot \int_{\Omega} \frac{\partial G}{\partial w}\left(\hat{\Phi}_{\mathrm{tr}}, \hat{W}\right) \psi_{i}(x) \psi_{j}(x) \mathrm{d} x=-\left\langle\frac{\partial r}{\partial w}\left(\hat{\Phi}_{\mathrm{tr}}, \hat{\Phi}_{e}, \hat{W}\right), \psi_{j}\right\rangle, \quad 0 \leqslant j \leqslant N ; \\
& p_{j, N}(0)=0 ; q_{j, N}(0)=0 \quad 0 \leqslant j \leqslant N .
\end{aligned}
$$

Specifying the data for (4.111)-(4.113) according to the Rogers-McCulloch model, the problem reads as

$$
\begin{aligned}
& \frac{\mathrm{d} p_{j, N}(s)}{\mathrm{d} s}+\lambda_{j} p_{j, N}(s)+\sum_{i=0}^{N} p_{i, N}(s) \cdot \int_{\Omega}\left(3 b\left(\hat{\Phi}_{\mathrm{tr}}\right)^{2}+2(a+1) b \hat{\Phi}_{\mathrm{tr}}+\hat{W}+a b\right) \psi_{i} \psi_{j} \mathrm{~d} x \\
& -\varepsilon \kappa q_{j, N}(s)=\left\langle\widetilde{S}(s), \psi_{j}\right\rangle, \quad 0 \leqslant j \leqslant N ; \\
& \frac{\mathrm{d} q_{j, N}(s)}{\mathrm{d} s}+\sum_{i=0}^{N} p_{i, N}(s) \cdot \int_{\Omega} \hat{\Phi}_{\mathrm{tr}} \psi_{i} \psi_{j} \mathrm{~d} x+\varepsilon q_{j, N}(s)=-\left\langle\frac{\partial r}{\partial w}\left(\hat{\Phi}_{\mathrm{tr}}, \hat{\Phi}_{e}, \hat{W}\right), \psi_{j}\right\rangle, \quad 0 \leqslant j \leqslant N ;(4.115) \\
& p_{j, N}(0)=0 ; q_{j, N}(0)=0 \quad 0 \leqslant j \leqslant N .
\end{aligned}
$$

Obviously, all integrals with respect to $x$ are well-defined and the coefficients as well as the right-hand sides are integrable with respect to $s$ at least. Then, by [23], page 92, Theorem II.4.6, the initial-value problem (4.114)(4.116) admits a unique solution $\left(p_{0, N}, \ldots, p_{N, N}, q_{0, N}, \ldots, q_{N, N}\right) \in\left(W^{1,1}(0, T)\right)^{2(N+1)}$. As a consequence of the orthogonality relations, $P_{1}^{N}$ and $P_{3}^{N}$ obey the equations

$$
\begin{array}{r}
\frac{\mathrm{d}}{\mathrm{d} s}\left\langle P_{1}^{N}(s), \psi\right\rangle+A\left(P_{1}^{N}(s), \psi\right)+\int_{\Omega}\left(\frac{\partial I_{\mathrm{ion}}}{\partial \varphi}\left(\hat{\Phi}_{\mathrm{tr}}, \hat{W}\right) P_{1}^{N}+\frac{\partial G}{\partial \varphi}\left(\hat{\Phi}_{\mathrm{tr}}, \hat{W}\right) P_{3}^{N}\right) \psi \mathrm{d} x \\
=\langle\widetilde{S}(s), \psi\rangle \quad \forall \psi \in \mathrm{X}^{N}(\Omega) ; \\
\frac{\mathrm{d}}{\mathrm{d} s}\left\langle P_{3}^{N}(s), \psi\right\rangle+\int_{\Omega}\left(\frac{\partial I_{\mathrm{ion}}}{\partial w}\left(\hat{\Phi}_{\mathrm{tr}}, \hat{W}\right) P_{1}^{N}+\frac{\partial G}{\partial w}\left(\hat{\Phi}_{\mathrm{tr}}, \hat{W}\right) P_{3}^{N}\right) \psi \mathrm{d} x \\
=-\left\langle\frac{\partial r}{\partial w}\left(\hat{\Phi}_{\mathrm{tr}}, \hat{\Phi}_{e}, \hat{W}\right), \psi\right\rangle \quad \forall \psi \in \mathrm{X}^{N}(\Omega) .
\end{array}
$$


In this sense, the functions $P_{1}^{N}, P_{3}^{N}$ can be interpreted as approximate solutions of the reduced adjoint system.

- Step 2. A priori estimates for the approximate solutions $P_{1}^{N}, P_{3}^{N}$. The functions $P_{1}^{N}, P_{3}^{N}$ obey the a priori estimates from Theorem 4.1,2). More precisely, the following holds:

Lemma 4.4. Let the assumptions of Theorem 4.1, 2), hold for the data of (P) and a feasible solution $\left(\hat{\Phi}_{\mathrm{tr}}, \hat{\Phi}_{e}\right.$, $\left.\hat{W}, \hat{I}_{e}\right)$ where $q=10 / 9$. Then for all $N \in \mathbb{N}_{0}$, the functions $P_{1}^{N}, P_{3}^{N}$ satisfy the estimate

$$
\begin{array}{r}
\left\|P_{1}^{N}\right\|_{C^{0}\left[[0, T], L^{2}(\Omega)\right]}^{2}+\left\|P_{1}^{N}\right\|_{L^{2}\left[(0, T), W^{1,2}(\Omega)\right]}^{2}+\left\|\partial P_{1}^{N} / \partial s\right\|_{L^{q}\left[(0, T),\left(W^{1,2}(\Omega)\right)^{*}\right]}^{q} \\
+\left\|P_{3}^{N}\right\|_{C^{0}\left[[0, T], L^{2}(\Omega)\right]}^{2}+\left\|\partial P_{3}^{N} / \partial s\right\|_{L^{q}\left[(0, T), L^{2}(\Omega)\right]}^{q} \leqslant C
\end{array}
$$

for a constant $C>0$ independent of $N$.

Proof. We rely on the Proof of Theorem 4.1. First, we observe that Lemma 4.3, (4.51) and (4.55) remain true if $P_{1}$ and $P_{3}$ are replaced by $P_{1}^{N}$ and $P_{3}^{N}$ since, in Steps 2 and 3 of the proof above, the reduced equations must be studied only for the special test functions $P_{1}^{N}(s), P_{3}^{N}(s) \in \mathrm{X}^{N}(\Omega)$. Further, we observe that

$$
\begin{aligned}
& \left\|\partial P_{1}^{N} / \partial s\right\|_{L^{q}\left[(0, T),\left(W^{1,2}(\Omega)\right)^{*}\right]}^{q}=\int_{0}^{T} \sup _{\|\psi\|_{W^{1,2}(\Omega)}=1}\left|\left\langle\partial P_{1}^{N}(s) / \partial s, \psi\right\rangle\right|^{q} \mathrm{~d} s \\
& =\int_{0}^{T} \sup _{\left\|\sum_{j=0}^{\infty} c_{j} \psi_{j}\right\| W^{1,2(\Omega)}=1}\left|\left\langle\sum_{i=0}^{N} \frac{\mathrm{d} p_{i, N}(s)}{\mathrm{d} s} \psi_{i}, \sum_{j=0}^{\infty} c_{j} \psi_{j}\right\rangle\right|^{q} \mathrm{~d} s \\
& =\int_{0}^{T} \sup _{\left\|\sum_{j=0}^{\infty} c_{j} \psi_{j}\right\| W^{1,2(\Omega)}=1}\left|\left\langle\sum_{i=0}^{N} \frac{\mathrm{d} p_{i, N}(s)}{\mathrm{d} s} \psi_{i}, \sum_{j=0}^{N} c_{j} \psi_{j}\right\rangle\right|^{q} \mathrm{~d} s \\
& =\int_{0}^{T} \sup _{\psi \in \mathrm{X}^{N},\|\psi\|_{W^{1,2}(\Omega)}=1}\left|\left\langle\partial P_{1}^{N}(s) / \partial s, \psi\right\rangle\right|^{q} \mathrm{~d} s .
\end{aligned}
$$

By (4.117), the calculations from the Proof of Theorem 4.1, Step 4, can be repeated now, resulting in a uniform bound for $\left\|\partial P_{1}^{N} / \partial s\right\|_{L^{q}\left[(0, T),\left(W^{1,2}(\Omega)\right)^{*}\right]}^{q}$. In the same manner, we may repeat the derivation from Step 5 since

$$
\begin{gathered}
\left\|\partial P_{3}^{N} / \partial s\right\|_{L^{q}\left[(0, T),\left(L^{2}(\Omega)\right)^{*}\right]}^{q}=\int_{0}^{T} \sup _{\|\psi\|_{L^{2}(\Omega)}=1}\left|\left\langle\partial P_{3}^{N}(s) / \partial s, \psi\right\rangle\right|^{q} \mathrm{~d} s \\
=\int_{0}^{T} \sup _{\psi \in \mathrm{X}^{N},\|\psi\|_{L^{2}(\Omega)}=1}\left|\left\langle\partial P_{3}^{N}(s) / \partial s, \psi\right\rangle\right|^{q} \mathrm{~d} s,
\end{gathered}
$$

and we obtain a uniform bound for $\left\|\partial P_{3}^{N} / \partial s\right\|_{L^{q}\left[(0, T), L^{2}(\Omega)\right]}^{q}$ as well. The arguments from Step 7 hold without alterations.

- Step 3. The solution for the reduced adjoint system. Lemma 4.4 implies that we may select a subsequence $\left\{\left(P_{1}^{N^{\prime}}, P_{3}^{N^{\prime}}\right)\right\}$ of $\left\{\left(P_{1}^{N}, P_{3}^{N}\right)\right\}$ with convergence to limit elements in the following sense:

$$
P_{1}^{N^{\prime}} \rightarrow L^{2}\left[(0, T), W^{1,2}(\Omega)\right] P_{1}
$$




$$
\begin{aligned}
& d P_{1}^{N^{\prime}} / \mathrm{d} s \rightarrow^{L^{q}}\left[(0, T),\left(W^{1,2}(\Omega)\right)^{*}\right] \widetilde{P} \\
& P_{3}^{N^{\prime}} \rightarrow L^{L^{2}}\left[(0, T), L^{2}(\Omega)\right] P_{3} ; \\
& d P_{3}^{N^{\prime}} / \mathrm{d} s \rightarrow^{L^{q}}\left[(0, T),\left(L^{2}(\Omega)\right)^{*}\right] \widetilde{Q} .
\end{aligned}
$$

Consequently, taking an arbitrary element $\psi_{j} \in W^{1,2}(\Omega)$ from the orthonormal base, we find

$$
\begin{gathered}
\left\langle\widetilde{P}(s), \psi_{j}\right\rangle+A\left(P_{1}(s), \psi_{j}\right)+\int_{\Omega}\left(\frac{\partial I_{\mathrm{ion}}}{\partial \varphi}\left(\hat{\Phi}_{\mathrm{tr}}, \hat{W}\right) P_{1}+\frac{\partial G}{\partial \varphi}\left(\hat{\Phi}_{\mathrm{tr}}, \hat{W}\right) P_{3}\right) \psi_{j} \mathrm{~d} x-\left\langle\widetilde{S}(s), \psi_{j}\right\rangle \\
=\lim _{N^{\prime} \rightarrow \infty}\left(\frac{\mathrm{d}}{\mathrm{d} s}\left\langle P_{1}^{N^{\prime}}(s), \psi_{j}\right\rangle+A\left(P_{1}^{N^{\prime}}(s), \psi_{j}\right)+\int_{\Omega}\left(\frac{\partial I_{\mathrm{ion}}}{\partial \varphi}\left(\hat{\Phi}_{\mathrm{tr}}, \hat{W}\right) P_{1}^{N^{\prime}}\right.\right. \\
\left.\left.+\frac{\partial G}{\partial \varphi}\left(\hat{\Phi}_{\mathrm{tr}}, \hat{W}\right) P_{3}^{N^{\prime}}\right) \psi_{j} \mathrm{~d} x-\left\langle\widetilde{S}(s), \psi_{j}\right\rangle\right)=0
\end{gathered}
$$

since $\psi_{j} \in \mathrm{X}^{N^{\prime}}$ for all sufficiently large $N^{\prime} \in \mathbb{N}$. For the same reason, it holds that

$$
\begin{aligned}
\langle\widetilde{Q}(s), & \left.\psi_{j}\right\rangle+\int_{\Omega}\left(\frac{\partial I_{\mathrm{ion}}}{\partial w}\left(\hat{\Phi}_{\mathrm{tr}}, \hat{W}\right) P_{1}+\frac{\partial G}{\partial w}\left(\hat{\Phi}_{\mathrm{tr}}, \hat{W}\right) P_{3}\right) \psi_{j} \mathrm{~d} x+\left\langle\frac{\partial r}{\partial w}\left(\hat{\Phi}_{\mathrm{tr}}, \hat{\Phi}_{e}, \hat{W}\right), \psi_{j}\right\rangle \\
= & \lim _{N^{\prime} \rightarrow \infty}\left(\frac{\mathrm{d}}{\mathrm{d} s}\left\langle P_{3}^{N^{\prime}}(s), \psi_{j}\right\rangle+\int_{\Omega}\left(\frac{\partial I_{\mathrm{ion}}}{\partial w}\left(\hat{\Phi}_{\mathrm{tr}}, \hat{W}\right) P_{1}^{N^{\prime}}+\frac{\partial G}{\partial w}\left(\hat{\Phi}_{\mathrm{tr}}, \hat{W}\right) P_{3}^{N^{\prime}}\right) \psi_{j} \mathrm{~d} x\right. \\
& \left.+\left\langle\frac{\partial r}{\partial w}\left(\hat{\Phi}_{\mathrm{tr}}, \hat{\Phi}_{e}, \hat{W}\right), \psi_{j}\right\rangle\right)=0 .
\end{aligned}
$$

Weak continuity of the distributional differential operator implies that $\widetilde{P}=d P_{1} / \mathrm{d} s$ and $\widetilde{Q}=d P_{3} / \mathrm{d} s$ in the sense of distributions. Further, it obviously holds that $P_{1}(x, 0)=\lim _{N^{\prime} \rightarrow \infty} P_{1}^{N}(x, 0)=0$ and $P_{3}(x, 0)=$ $\lim _{N^{\prime} \rightarrow \infty} P_{3}^{N}(x, 0)=0$. Since $\left\{\psi_{i}\right\}$ lies dense in $W^{1,2}(\Omega)$ as well as in $L^{2}(\Omega)$, the functions $P_{1}$ and $P_{3}$ form a weak solution of the reduced adjoint system.

- Step 4. Completion of the adjoint solution. As indicated in Section 4.2., the solution $\left(P_{1}, P_{3}\right)$ of the reduced adjoint system may be completed to a weak solution $\left(P_{1}, P_{2}, P_{3}\right)$ of the adjoint system where $P_{2} \in$ $L^{2}\left[(0, T), W^{1,2}(\Omega)\right]$ with $\int_{\Omega} P_{2}(x, s) \mathrm{d} x=0(\forall) s \in(0, T)$ is uniquely determined by $P_{1}, P_{3}$. The claimed regularity of the solution is guaranteed by Theorem 4.1,2).

- Step 5. Uniqueness. Since the reduced adjoint system is linear with respect to $P_{1}$ and $P_{3}$, estimate (4.20) yields the uniqueness of its weak solution $\left(P_{1}, P_{3}\right)$ within the space $\left(L^{\infty}\left[(0, T), L^{2}(\Omega)\right] \cap L^{2}[(0, T)\right.$, $\left.\left.W^{1,2}(\Omega)\right]\right) \times L^{\infty}\left[(0, T), L^{2}(\Omega)\right]$. The completion of $\left(P_{1}, P_{3}\right)$ to a weak solution $\left(P_{1}, P_{2}, P_{3}\right)$ of the adjoint system is uniquely determined as well. This finishes the Proof of Theorem 4.2.

Remark 4.5. 1) If the Rogers-McCulloch model in (3.12)-(3.14) is replaced by the FitzHugh-Nagumo model then the Proofs of Theorems 4.1. and 4.2. can be repeated with only minor alterations.

2) Theorems 4.1. and 4.2. remain even true if (3.12)-(3.14) is considered with the linearized Aliev-Panfilov model. In the proofs, we must work with

$$
\frac{\partial G}{\partial \varphi}\left(\hat{\Phi}_{\mathrm{tr}}, \hat{W}\right)=-\varepsilon \kappa(a+1)+2 \varepsilon \kappa \hat{\Phi}_{\mathrm{tr}}
$$


instead of $\partial G\left(\hat{\Phi}_{\mathrm{tr}}, \hat{W}\right) / \partial \varphi \equiv-\varepsilon \kappa$. Thus the estimations (4.36)-(4.55) have to be modified in the following way: On the right-hand side of (4.36), the term $\varepsilon \kappa \int_{\Omega}\left|P_{1} P_{3}\right| \mathrm{d} x$ must be replaced by

$$
\varepsilon \kappa(a+1) \int_{\Omega}\left|P_{1} P_{3}\right| \mathrm{d} x+\varepsilon \kappa \int_{\Omega}\left|\hat{\Phi}_{\mathrm{tr}} P_{1} P_{3}\right| \mathrm{d} x .
$$

The estimation of the first member of (4.133) runs as above, for the second one we get with arbitrary $\varepsilon_{3}(s)>0$ :

$$
\begin{aligned}
\int_{\Omega}\left|\hat{\Phi}_{\mathrm{tr}} P_{1} P_{3}\right| \mathrm{d} x \leqslant C & \varepsilon_{3}(s) \int_{\Omega}\left|\hat{\Phi}_{\mathrm{tr}} P_{1}\right|^{2} \mathrm{~d} x+\frac{C}{\varepsilon_{3}(s)}\left\|P_{3}\right\|_{L^{2}(\Omega)}^{2} \\
& \leqslant C \varepsilon_{3}(s)\left\|\hat{\Phi}_{\mathrm{tr}}\right\|_{L^{4}(\Omega)}^{2} \cdot\left\|P_{1}\right\|_{L^{4}(\Omega)}^{2}+\frac{C}{\varepsilon_{3}(s)}\left\|P_{3}\right\|_{L^{2}(\Omega)}^{2} .
\end{aligned}
$$

We choose $\varepsilon_{3}(s)=\varepsilon_{3}^{\prime} /\left(1+\left\|\hat{\Phi}_{\mathrm{tr}}\right\|_{L^{4}(\Omega)}^{2}\right)$ with $\varepsilon_{3}^{\prime}>0$, thus getting

$$
\int_{\Omega}\left|\hat{\Phi}_{\mathrm{tr}} P_{1} P_{3}\right| \mathrm{d} x \leqslant C \varepsilon^{\prime}\left\|P_{1}\right\|_{W^{1,2}(\Omega)}^{2}+\frac{C}{\varepsilon_{3}^{\prime}}\left(1+\left\|\hat{\Phi}_{\mathrm{tr}}\right\|_{L^{4}(\Omega)}^{2}\right)\left\|P_{3}\right\|_{L^{2}(\Omega)}^{2},
$$

and with appropriate choices of $\varepsilon_{3}^{\prime}>0$, we may proceed as above. Further alterations concern the estimations (4.58)-(4.88). In (4.58), the term $\sup _{\ldots} \varepsilon^{q} \kappa^{q}\left(\int_{\Omega}\left|P_{3}\right||\psi| \mathrm{d} x\right)^{q}$ must be replaced by

$$
\sup _{\ldots} \varepsilon^{q} \kappa^{q}(a+1)^{q}\left(\int_{\Omega}\left|P_{3}\right||\psi| \mathrm{d} x\right)^{q}+\sup _{\ldots} \varepsilon^{q} \kappa^{q}\left(\int_{\Omega}\left|\hat{\Phi}_{\mathrm{tr}} P_{3} \psi\right| \mathrm{d} x\right)^{q} .
$$

Despite of the lesser regularity of $\hat{W}$ for the linearized Aliev-Panfilov model, the estimations (4.70)-(4.80) can be maintained since the solution satisfies $\hat{W} \in C^{0}\left[[0, T], L^{8 / 3}(\Omega)\right] \hookrightarrow L^{10 / 4}\left[(0, T), L^{20 / 11}(\Omega)\right]$. In $(4.81)-$ (4.84), we must add an estimate for the second term from (4.137). Consider therefore

$$
\begin{aligned}
& \sup _{\ldots} \int_{0}^{T} \int_{\Omega}\left|\hat{\Phi}_{\operatorname{tr}}\right|^{10 / 9}\left|P_{3}\right|^{10 / 9}|\psi|^{10 / 9} \mathrm{~d} x \mathrm{~d} s \\
\leqslant & C \sup _{\ldots} \int_{0}^{T}\left(\int_{\Omega}\left|\hat{\Phi}_{\mathrm{tr}}\right|^{5} \mathrm{~d} x\right)^{4 / 18}\left(\int_{\Omega}|\psi|^{5} \mathrm{~d} x\right)^{4 / 18}\left(\int_{\Omega}\left|P_{3}\right|^{2} \mathrm{~d} x\right)^{10 / 18} \mathrm{~d} s \\
\leqslant & C \sup _{\ldots} \int_{0}^{T}\left(\left\|\hat{\Phi}_{\mathrm{tr}}\right\|_{L^{5}(\Omega)}^{10 / 9} \cdot\|\psi\|_{W^{1,2}(\Omega)}^{10 / 9} \cdot\left\|P_{3}\right\|_{L^{2}(\Omega)}^{10 / 9}\right) \mathrm{d} s=C \int_{0}^{T}\left(\left\|\hat{\Phi}_{\mathrm{tr}}\right\|_{L^{5}(\Omega)}^{10 / 9} \cdot\left\|P_{3}\right\|_{L^{2}(\Omega)}^{10 / 9}\right) \mathrm{d} s( \\
\leqslant & \left.C\left\|\hat{\Phi}_{\operatorname{tr}}\right\|_{L^{10 / 9}\left[(0, T), L^{5}(\Omega)\right]}^{10 / 9} \cdot\left\|P_{3}\right\|_{L^{\infty}\left[(0, T), L^{2}(\Omega)\right.}^{10 / 9}\right]
\end{aligned}
$$

and we may proceed as above. The other parts of the Proof of Theorem 4.1. as well as the Proof of Theorem 4.2. remain unchanged.

\section{NeCESSARY OPtimality CONDitions}

\subsection{Statement of the theorems}

Definition 5.1 (Weak local minimizer). A quadruple $\left(\hat{\Phi}_{\mathrm{tr}}, \hat{\Phi}_{e}, \hat{W}, \hat{I}_{e}\right)$, which is feasible in $(\mathrm{P})$, is called a weak local minimizer of $(\mathrm{P})$ iff there exists a number $\varepsilon>0$ such that for all admissible $\left(\Phi_{\mathrm{tr}}, \Phi_{e}, W, I_{e}\right)$ the conditions

$$
\left\|\Phi_{\mathrm{tr}}-\hat{\Phi}_{\mathrm{tr}}\right\|_{\mathrm{X}_{1}} \leqslant \varepsilon,\left\|\Phi_{e}-\hat{\Phi}_{e}\right\|_{\mathrm{X}_{2}} \leqslant \varepsilon,\|W-\hat{W}\|_{\mathrm{X}_{3}} \leqslant \varepsilon,\left\|I_{e}-\hat{I}_{e}\right\|_{\mathrm{X}_{4}} \leqslant \varepsilon
$$

imply the relation $F\left(\hat{\Phi}_{\mathrm{tr}}, \hat{\Phi}_{e}, \hat{W}, \hat{I}_{e}\right) \leqslant F\left(\Phi_{\mathrm{tr}}, \Phi_{e}, W, I_{e}\right)$. 
The necessary optimality conditions for weak local minimizers of $(\mathrm{P})$ can be formulated as follows:

Theorem 5.2 (First-order necessary optimality conditions for the control problem $(\mathrm{P})$ ). We consider problem (P), (3.11)-(3.17), under the assumptions of Section 3.1. with the Rogers-McCulloch or the FitzHugh-Nagumo model. Assume further that 1) $\Omega \subset \mathbb{R}^{3}$ admits a $C^{1,1}$-boundary, 2) $M_{i}, M_{e}: \operatorname{cl}(\Omega) \rightarrow \mathbb{R}^{3 \times 3}$ are symmetric, positive definite matrix functions obeying $(2.5)$ with $W^{1, \infty}(\Omega)$-coefficients, and 3) the integrand $r(x, t, \varphi, \eta, w)$ is continuously differentiable with respect to $\varphi, \eta$ and $w$. Let $\left(\hat{\Phi}_{\mathrm{tr}}, \hat{\Phi}_{e}, \hat{W}, \hat{I}_{e}\right)$ be a weak local minimizer of $(\mathrm{P})$ such that $\left(\hat{\Phi}_{\mathrm{tr}}, \hat{\Phi}_{e}, \hat{W}\right)$ is a strong solution of the bidomain system on $[0, T], \hat{I}_{e} \in W^{1,2}\left[(0, T), L^{2}(\Omega)\right]$ and

$$
\frac{\partial r}{\partial \varphi}\left(\hat{\Phi}_{\mathrm{tr}}, \hat{\Phi}_{e}, \hat{W}\right), \frac{\partial r}{\partial \eta}\left(\hat{\Phi}_{\mathrm{tr}}, \hat{\Phi}_{e}, \hat{W}\right), \frac{\partial r}{\partial w}\left(\hat{\Phi}_{\mathrm{tr}}, \hat{\Phi}_{e}, \hat{W}\right) \in L^{2 q}\left[(0, T), L^{2}(\Omega)\right]
$$

where $q \geqslant 10 / 9$. Then there exist multipliers $P_{1} \in L^{4}\left[(0, T), W^{1,2}(\Omega)\right], P_{2} \in L^{2}\left[(0, T), W^{1,2}(\Omega)\right] \cap$ $\left\{Z \mid \int_{\Omega} Z(x, t) \mathrm{d} x=0(\forall) t \in(0, T)\right\}$ and $P_{3} \in L^{2}\left(\Omega_{T}\right)$, satisfying together with $\left(\hat{\Phi}_{\mathrm{tr}}, \hat{\Phi}_{e}, \hat{W}, \hat{I}_{e}\right)$ the adjoint equations (4.8)-(4.10), which are solved in weak sense, as well as the optimality condition

$$
\int_{0}^{T} \int_{\Omega_{\mathrm{con}}}\left(\mu \hat{I}_{e}-Q P_{2}\right) \cdot\left(I_{e}-\hat{I}_{e}\right) \mathrm{d} x \mathrm{~d} t \geqslant 0 \quad \forall I_{e} \in \mathcal{C} .
$$

If the linearized Aliev-Panfilov model is specified then all assertions remain true provided that $\partial r\left(\hat{\Phi}_{\mathrm{tr}}, \hat{\Phi}_{e}, \hat{W}\right) /$ dw belongs to $L^{3}\left(\Omega_{T}\right)$ instead of $L^{2}\left(\Omega_{T}\right)$.

The assumptions of Theorem 5.2. reflect the fact that there is a regularity gap between the weak solutions of the primal and adjoint equations. The duality pairing between $\partial \Phi_{\operatorname{tr}} / \partial t \in L^{4 / 3}\left[(0, T),\left(W^{1,2}(\Omega)\right)^{*}\right]$ and $P_{1} \in L^{2}\left[(0, T), W^{1,2}(\Omega)\right]$ is not well-defined, and hence further regularity is required. In order to gain this regularity, we have to impose that $\left(\hat{\Phi}_{\mathrm{tr}}, \hat{\Phi}_{e}, \hat{W}\right)$ is a strong solution of the bidomain system rather than a weak one. Sufficient conditions for strong local solvability of (3.12)-(3.14) may be found in [22].

Corollary 5.3 (Pointwise formulation of the optimality condition). Under the assumptions of Theorem 5.2., let the optimal control $\hat{I}_{e}$ be represented as $\hat{I}_{e}=Q \hat{I}$ with $\hat{I} \in L^{\infty}\left[(0, T), L^{2}(\Omega)\right]$, $\operatorname{supp}(\hat{I}) \subseteq \Omega_{\text {con }} \times[0, T]$ and $|\hat{I}(x, t)| \leqslant R$ for almost all $(x, t) \in \Omega_{T}$. The optimality condition (5.3) from Theorem 5.2. then implies the following Pontryagin minimum condition, which holds a.e. pointwise:

$$
\begin{array}{r}
\hat{I}\left(x_{0}, t_{0}\right) \cdot\left(\mu \cdot Q \hat{I}\left(x_{0}, t_{0}\right)-Q P_{2}\left(x_{0}, t_{0}\right)\right)=\underset{-R \leqslant \eta \leqslant R}{\operatorname{Min}} \eta\left(\mu \cdot Q \hat{I}\left(x_{0}, t_{0}\right)-Q P_{2}\left(x_{0}, t_{0}\right)\right) \\
(\forall)\left(x_{0}, t_{0}\right) \in \Omega_{\text {con }} \times[0, T] .
\end{array}
$$

Consequently, for a.e. $(x, t) \in \Omega_{\mathrm{con}} \times[0, T]$ the following implications hold:

$$
\begin{aligned}
& Q \hat{I}(x, t)-\frac{1}{\mu} Q P_{2}(x, t)>0 \Longrightarrow \hat{I}(x, t)=-R ; \\
& Q \hat{I}(x, t)-\frac{1}{\mu} Q P_{2}(x, t)<0 \Longrightarrow \hat{I}(x, t)=R \text { and } \\
& \hat{I}(x, t) \in(-R, R) \Longrightarrow Q \hat{I}(x, t)-\frac{1}{\mu} Q P_{2}(x, t)=0 .
\end{aligned}
$$

Corollary 5.4 (Regularity of weak local minimizers). Under the assumptions of Theorem 5.2., consider a weak local minimizer $\left(\hat{\Phi}_{\mathrm{tr}}, \hat{\Phi}_{e}, \hat{W}, \hat{I}_{e}\right)$ of $(\mathrm{P})$, whose control part $\hat{I}_{e}=Q \hat{I}$ is generated by a function $\hat{I}$ with $|\hat{I}(x, t)|<R$ a.e. Then $\hat{I}_{e} \mid \Omega_{\text {con }}$ belongs to the space $L^{\infty}\left(\Omega_{\text {con }} \times[0, T]\right) \cap L^{2}\left[(0, T), W^{1,2}\left(\Omega_{\text {con }}\right)\right]$ 
Fur numerical purposes, it is useful to specify the Gâteaux derivative of the reduced cost functional $\widetilde{F}: \mathcal{C} \rightarrow$ $\mathbb{R}$. It is defined through

$$
\widetilde{F}\left(I_{e}\right)=F\left(\Phi_{\operatorname{tr}}\left(I_{e}\right), \Phi_{e}\left(I_{e}\right), W\left(I_{e}\right), I_{e}\right)
$$

with the aid of the control-to-state mapping $I_{e} \longmapsto\left(\Phi_{\mathrm{tr}}\left(I_{e}\right), \Phi_{e}\left(I_{e}\right), W\left(I_{e}\right)\right)$, which is well-defined by Proposition 3.1.

Corollary 5.5 (First variation of the reduced cost functional). Under the assumptions of Theorem 5.2., the Gâteaux derivative of the reduced cost functional $\widetilde{F}$ at $\hat{I}_{e} \in \mathcal{C}$ is given through

$$
D_{I_{e}} \widetilde{F}\left(\hat{I}_{e}\right)=\mu \hat{I}_{e}-Q P_{2}\left(\hat{I}_{e}\right)
$$

where $\left(P_{1}\left(\hat{I}_{e}\right), P_{2}\left(\hat{I}_{e}\right), P_{3}\left(\hat{I}_{e}\right)\right)$ denotes the solution of the adjoint system (4.8)-(4.10) corresponding to $\left(\Phi_{\operatorname{tr}}\left(\hat{I}_{e}\right), \Phi_{e}\left(\hat{I}_{e}\right), W\left(\hat{I}_{e}\right), \hat{I}_{e}\right)$.

\subsection{Proof of the necessary optimality conditions}

Proof of Theorem 5.2. As mentioned in the introduction, the proof of the necessary optimality conditions for (P) is based on the stability estimate for the bidomain system (Thm. 2.4) and the existence theorem for the adjoint system (Thm. 4.2), which will be invoked in Steps 2 and 3 of the proof, respectively.

- Step 1. Variation of the weak local minimizer in a feasible direction. Assume that $\left(\hat{\Phi}_{\mathrm{tr}}, \hat{\Phi}_{e}, \hat{W}, \hat{I}_{e}\right)$ is a weak local minimizer of $(\mathrm{P})$. If $I_{e} \in \mathcal{C}$ is an arbitrary feasible control with $\left\|I_{e}-\hat{I}_{e}\right\|_{L^{\infty}\left[(0, T), L^{2}(\Omega)\right]} \leqslant \varepsilon$ then, by Proposition 3.2., all controls

$$
I_{e}(s)=\hat{I}_{e}+s\left(I_{e}-\hat{I}_{e}\right), 0 \leqslant s \leqslant 1,
$$

belong to $\mathcal{C}$ as well. By Proposition 3.1., for every $I_{e}(s) \in L^{\infty}\left[(0, T), L^{2}(\Omega)\right]$, there exists a corresponding weak solution $\left(\Phi_{\mathrm{tr}}(s), \Phi_{e}(s), W(s)\right) \in \mathrm{X}_{1} \times \mathrm{X}_{2} \times \mathrm{X}_{3}$ for the bidomain system on $[0, T]$. Thus the quadruples $\left(\Phi_{\operatorname{tr}}(s), \Phi_{e}(s), W(s), I_{e}(s)\right)$ are feasible in $(\mathrm{P})$ for all $0 \leqslant s \leqslant 1$. On the other hand, from [12], page 7, Theorem 2.7, it follows that every feasible solution of $(\mathrm{P})$ within a closed ball

$$
\mathrm{U}_{\varepsilon}\left(\hat{\Phi}_{\mathrm{tr}}, \hat{\Phi}_{e}, \hat{W}, \hat{I}_{e}\right)=\mathrm{K}\left(\hat{\Phi}_{\mathrm{tr}}, C \varepsilon\right) \times \mathrm{K}\left(\hat{\Phi}_{e}, C \varepsilon\right) \times \mathrm{K}(\hat{W}, C \varepsilon) \times \mathrm{K}\left(\hat{I}_{e}, \varepsilon\right) \subset \mathrm{X}_{1} \times \mathrm{X}_{2} \times \mathrm{X}_{3} \times \mathrm{X}_{4}
$$

can be generated in this way.

\section{- Step 2.}

Lemma 5.6. For all $I_{e} \in \mathcal{C},\left\|I_{e}-\hat{I}_{e}\right\|_{L^{\infty}\left[(0, T), L^{2}(\Omega)\right]} \leqslant \varepsilon$ implies that

$$
\begin{aligned}
& \lim _{s \rightarrow 0+0} \frac{1}{s}\left\|\Phi_{\operatorname{tr}}(s)-\hat{\Phi}_{\mathrm{tr}}\right\|_{\mathrm{X}_{1}}^{2}=0 ; \quad \lim _{s \rightarrow 0+0} \frac{1}{s}\left\|\Phi_{\operatorname{tr}}(s)-\hat{\Phi}_{\mathrm{tr}}\right\|_{\tilde{\mathrm{X}}_{1}}^{2}=0 ; \\
& \lim _{s \rightarrow 0+0} \frac{1}{s}\left\|\Phi_{e}(s)-\hat{\Phi}_{e}\right\|_{\mathrm{X}_{2}}^{2}=0 ; \\
& \lim _{s \rightarrow 0+0} \frac{1}{s}\|W(s)-\hat{W}\|_{\mathrm{X}_{3}}^{2}=0 \text { and } \lim _{s \rightarrow 0+0} \frac{1}{s}\|W(s)-\hat{W}\|_{\widetilde{\mathrm{X}}_{3}}^{2}=0 .
\end{aligned}
$$

Proof. The stability estimate [12], page 7, Theorem 2.7, (2.38), implies

$$
\left\|\Phi_{\operatorname{tr}}(s)-\hat{\Phi}_{\operatorname{tr}}\right\|_{\mathrm{X}_{1}}^{2}=\left\|\Phi_{\operatorname{tr}}(s)-\hat{\Phi}_{\operatorname{tr}}\right\|_{L^{2}\left[(0, T), W^{1,2}(\Omega)\right.}^{2} \leqslant C \cdot\left\|I_{e}(s)-\hat{I}_{e}\right\|_{L^{\infty}\left[(0, T),\left(W^{1,2}(\Omega)\right)^{*}\right]}^{2}
$$




$$
\begin{gathered}
\leqslant C \cdot\left\|I_{e}(s)-\hat{I}_{e}\right\|_{L^{\infty}\left[(0, T), L^{2}(\Omega)\right]}^{2}=C s^{2}\left\|I_{e}-\hat{I}_{e}\right\|_{L^{\infty}\left[(0, T), L^{2}(\Omega)\right]}^{2} \Longrightarrow \\
\lim _{s \rightarrow 0+0} \frac{1}{s}\left\|\Phi_{\operatorname{tr}}(s)-\hat{\Phi}_{\operatorname{tr}}\right\|_{\mathrm{X}_{1}}^{2} \leqslant \lim _{s \rightarrow 0+0} C s\left\|I_{e}-\hat{I}_{e}\right\|_{L^{\infty}\left[(0, T), L^{2}(\Omega)\right]}^{2}=0
\end{gathered}
$$

as well as

$$
\begin{aligned}
&\left\|\Phi_{e}(s)-\hat{\Phi}_{e}\right\|_{\mathrm{X}_{2}}^{2}=\left\|\Phi_{e}(s)-\hat{\Phi}_{e}\right\|_{L^{2}\left[(0, T), W^{1,2}(\Omega)\right]}^{2} \leqslant C \cdot\left\|I_{e}(s)-\hat{I}_{e}\right\|_{L^{\infty}\left[(0, T),\left(W^{1,2}(\Omega)\right)^{*}\right]}^{2} \\
& \leqslant C \cdot\left\|I_{e}(s)-\hat{I}_{e}\right\|_{L^{\infty}\left[(0, T), L^{2}(\Omega)\right]}^{2}=C s^{2}\left\|I_{e}-\hat{I}_{e}\right\|_{L^{\infty}\left[(0, T), L^{2}(\Omega)\right]}^{2} \Longrightarrow \\
& \lim _{s \rightarrow 0+0} \frac{1}{s}\left\|\Phi_{e}(s)-\hat{\Phi}_{e}\right\|_{\mathrm{X}_{2}}^{2} \leqslant \lim _{s \rightarrow 0+0} C s\left\|I_{e}-\hat{I}_{e}\right\|_{L^{\infty}\left[(0, T), L^{2}(\Omega)\right]}^{2}=0
\end{aligned}
$$

and

$$
\begin{gathered}
\|W(s)-\hat{W}\|_{\mathrm{X}_{3}}^{2}=\|W(s)-\hat{W}\|_{L^{2}\left(\Omega_{T}\right)}^{2} \leqslant C \cdot\left\|I_{e}(s)-\hat{I}_{e}\right\|_{L^{\infty}\left[(0, T),\left(W^{1,2}(\Omega)\right)^{*}\right]}^{2} \\
\left.\leqslant C \cdot\left\|I_{e}(s)-\hat{I}_{e}\right\|_{L^{\infty}\left[(0, T), L^{2}(\Omega)\right]}^{2}=C s^{2}\left\|I_{e}-\hat{I}_{e}\right\|_{L^{\infty}\left[(0, T), L^{2}(\Omega)\right]}^{2}\right] \\
\lim _{s \rightarrow 0+0} \frac{1}{s}\|W(s)-\hat{W}\|_{\mathrm{X}_{3}}^{2} \leqslant \lim _{s \rightarrow 0+0} C s\left\|I_{e}-\hat{I}_{e}\right\|_{L^{\infty}\left[(0, T), L^{2}(\Omega)\right]}^{2}=0 .
\end{gathered}
$$

In an analogous manner, the relation with $\|W(s)-\hat{W}\|_{\tilde{\mathrm{X}}_{3}}^{2}$ can be confirmed. In order to establish the relation with $\left\|\Phi_{\operatorname{tr}}(s)-\hat{\Phi}_{\operatorname{tr}}\right\|_{\tilde{\mathrm{X}}_{1}}^{2}$, we rely on [12], page 7, Theorem 2.7, (2.39), which leads to

$$
\begin{aligned}
& \left\|\Phi_{\operatorname{tr}}(s)-\hat{\Phi}_{\mathrm{tr}}\right\|_{\tilde{\mathrm{X}}_{1}}^{2}=\left\|\Phi_{\mathrm{tr}}(s)-\hat{\Phi}_{\mathrm{tr}}\right\|_{W^{1,4 / 3}\left[(0, T),\left(W^{1,2}(\Omega)\right)^{*}\right]}^{2} \\
& \leqslant C^{2} \cdot \operatorname{Max}\left(\left\|I_{e}(s)-\hat{I}_{e}\right\|_{L^{\infty}\left[(0, T),\left(W^{1,2}(\Omega)\right)^{*}\right]}^{2},\left\|I_{e}(s)-\hat{I}_{e}\right\|_{L^{\infty}\left[(0, T),\left(W^{1,2}(\Omega)\right)^{*}\right]}^{4}\right) \\
& \leqslant C \cdot \operatorname{Max}\left(s^{2}\left\|I_{e}-\hat{I}_{e}\right\|_{L^{\infty}\left[(0, T), L^{2}(\Omega)\right]}^{2}, s^{4}\left\|I_{e}-\hat{I}_{e}\right\|_{L^{\infty}\left[(0, T), L^{2}(\Omega)\right]}^{4}\right) \Longrightarrow \\
& \lim _{s \rightarrow 0+0} \frac{1}{s}\left\|\Phi_{\mathrm{tr}}(s)-\hat{\Phi}_{\mathrm{tr}}\right\|_{\tilde{X}_{1}}^{2} \\
& \leqslant \lim _{s \rightarrow 0+0} C \cdot \operatorname{Max}\left(s\left\|I_{e}-\hat{I}_{e}\right\|_{L^{\infty}\left[(0, T), L^{2}(\Omega)\right]}^{2}, s^{3}\left\|I_{e}-\hat{I}_{e}\right\|_{L^{\infty}\left[(0, T), L^{2}(\Omega)\right]}^{4}\right)
\end{aligned}
$$

- Step 3. By Theorems 4.1 and 4.2 , in correspondence to $\left(\hat{\Phi}_{\mathrm{tr}}, \hat{\Phi}_{e}, \hat{W}, \hat{I}_{e}\right)$, there exist functions $P_{1} \in$ $\left(L^{4 / 3}\left[(0, T),\left(W^{1,2}(\Omega)\right)^{*}\right]\right)^{*}=L^{4}\left[(0, T), W^{1,2}(\Omega)\right], P_{2} \in\left(L^{2}\left[(0, T), W^{1,2}(\Omega)\right]\right)^{*}=L^{2}[(0, T)$, $\left.W^{1,2}(\Omega)\right]$ with $\int_{\Omega} P_{2}(x, t) \mathrm{d} x=0$ for almost all $t \in(0, T)$ and $P_{3} \in\left(L^{2}\left[(0, T),\left(L^{2}(\Omega)\right)^{*}\right]\right)^{*}=L^{2}\left(\Omega_{T}\right)$ satisfying the system (4.8)-(4.10) as weak solutions. Consequently, $P_{1}, P_{2}$ and $P_{3}$ solve the adjoint 
equations (4.5)-(4.7) together with $P_{4}=-P_{1}(\cdot, 0)$ and $P_{5}=-P_{3}(\cdot, 0)$. With these functions, we may derive the following estimates:

Lemma 5.7. The following estimates hold true:

$$
\begin{gathered}
\lim _{s \rightarrow 0+0} \frac{1}{s}\left\langle P_{1}, D_{\Phi_{\mathrm{tr}}} E_{1}\left(\hat{\Phi}_{\mathrm{tr}}, \hat{\Phi}_{e}, \hat{W}\right)\left(\Phi_{\mathrm{tr}}(s)-\hat{\Phi}_{\mathrm{tr}}\right)+D_{\Phi_{e}}\right. \\
E_{1}\left(\hat{\Phi}_{\mathrm{tr}}, \hat{\Phi}_{e}, \hat{W}\right)\left(\Phi_{e}(s)-\hat{\Phi}_{e}\right) \\
\left.+D_{W} E_{1}\left(\hat{\Phi}_{\mathrm{tr}}, \hat{\Phi}_{e}, \hat{W}\right)(W(s)-\hat{W})\right\rangle=0 ; \\
\lim _{s \rightarrow 0+0} \frac{1}{s}\left\langle P_{2}, D_{\Phi_{\mathrm{tr}}} E_{2}\left(\hat{\Phi}_{\mathrm{tr}}, \hat{\Phi}_{e}, \hat{I}_{e}\right)\left(\Phi_{\mathrm{tr}}(s)-\hat{\Phi}_{\mathrm{tr}}\right)+D_{\Phi_{e}} E_{2}\left(\hat{\Phi}_{\mathrm{tr}}, \hat{\Phi}_{e}, \hat{I}_{e}\right)\left(\Phi_{e}(s)-\hat{\Phi}_{e}\right)\right. \\
+\left\langle P_{2}, D_{I_{e}} E_{2}\left(\hat{\Phi}_{\mathrm{tr}}, \hat{\Phi}_{e}, \hat{I}_{e}\right)\left(I_{e}-\hat{I}_{e}\right)\right\rangle=0 ; \\
\lim _{s \rightarrow 0+0} \frac{1}{s}\left\langle P_{3}, D_{\Phi_{\mathrm{tr}}} E_{3}\left(\hat{\Phi}_{\mathrm{tr}}, \hat{W}\right)\left(\Phi_{\mathrm{tr}}(s)-\hat{\Phi}_{\mathrm{tr}}\right)+D_{W} E_{3}\left(\hat{\Phi}_{\mathrm{tr}}, \hat{W}\right)(W(s)-\hat{W})\right\rangle=0 .
\end{gathered}
$$

Proof. We restrict ourselves to the proof of (5.23), noting that (5.24) and (5.25) can be confirmed in a completely analogous manner. Due to our assumptions on the differentiability of $r$, the principal theorem of calculus in its Bochner integral version is applicable, $c f$. [3], page 68, (2.1.11). For the feasible solutions $\left(\Phi_{\operatorname{tr}}(s), \Phi_{e}(s), W(s), I_{e}(s)\right)$ and $\left(\hat{\Phi}_{\mathrm{tr}}, \hat{\Phi}_{e}, \hat{W}, \hat{I}_{e}\right)$, we get from the first state equation in $(\mathrm{P}),(3.12)$ :

$$
\begin{aligned}
& \mathcal{o}=E_{1}\left(\Phi_{\mathrm{tr}}(s), \Phi_{e}(s), W(s)\right)-E_{1}\left(\hat{\Phi}_{\mathrm{tr}}, \hat{\Phi}_{e}, \hat{W}\right)=\int_{0}^{1} D_{\left(\Phi_{\mathrm{tr}}, \Phi_{e}, W\right)} E_{1}\left(\hat{\Phi}_{\mathrm{tr}}+\tau\left(\Phi_{\mathrm{tr}}(s)-\hat{\Phi}_{\mathrm{tr}}\right)\right. \\
& \left.\hat{\Phi}_{e}+\tau\left(\Phi_{e}(s)-\hat{\Phi}_{e}\right), \hat{W}+\tau(W(s)-\hat{W})\right)\left(\Phi_{\mathrm{tr}}(s)-\hat{\Phi}_{\mathrm{tr}}, \Phi_{e}(s)-\hat{\Phi}_{e}, W(s)-\hat{W}\right) \mathrm{d} \tau \Longrightarrow \\
& 0=\left\langle P_{1}, \int_{0}^{1}\left(D_{\left(\Phi_{\mathrm{tr}}, \Phi_{e}, W\right)} E_{1}\left(\hat{\Phi}_{\mathrm{tr}}+\tau\left(\Phi_{\mathrm{tr}}(s)-\hat{\Phi}_{\mathrm{tr}}\right), \hat{\Phi}_{e}+\tau\left(\Phi_{e}(s)-\hat{\Phi}_{e}\right), \hat{W}+\tau(W(s)-\hat{W})\right)\right.\right. \\
& \left.-D_{\left(\Phi_{\mathrm{tr}}, \Phi_{e}, W\right)} E_{1}\left(\hat{\Phi}_{\mathrm{tr}}, \hat{\Phi}_{e}, \hat{W}\right)\left(\Phi_{\mathrm{tr}}(s)-\hat{\Phi}_{\mathrm{tr}}, \Phi_{e}(s)-\hat{\Phi}_{e}, W(s)-\hat{\Phi}_{e}(s)\right) \mathrm{d} \tau\right\rangle \\
& +\left\langle P_{1}, D_{\left(\Phi_{\mathrm{tr}}, \Phi_{e}, W\right)} E_{1}\left(\hat{\Phi}_{\mathrm{tr}}, \hat{\Phi}_{e}, \hat{W}\right)\left(\hat{\Phi}_{\mathrm{tr}}(s)-\hat{\Phi}_{\mathrm{tr}}, \Phi_{e}(s)-\hat{\Phi}_{e}, W(s)-\hat{W}\right)\right\rangle \\
& =\left\langle P_{1}, \int_{0}^{1}\left(D_{\Phi_{\mathrm{tr}}} E_{1}\left(\hat{\Phi}_{\mathrm{tr}}+\tau \ldots, \hat{\Phi}_{e}+\tau \ldots, \hat{W}+\tau \ldots\right)\left(\Phi_{\mathrm{tr}}(s)-\hat{\Phi}_{\mathrm{tr}}\right)-D_{\Phi_{\mathrm{tr}}} E_{1}\left(\hat{\Phi}_{\mathrm{tr}}, \hat{\Phi}_{e}, \hat{W}\right)\left(\Phi_{\mathrm{tr}}(s)-\hat{\Phi}_{\mathrm{tr}}\right)\right.\right. \\
& +D_{\Phi_{e}} E_{1}\left(\hat{\Phi}_{\mathrm{tr}}+\tau \ldots, \hat{\Phi}_{e}+\tau \ldots, \hat{W}+\tau \ldots\right)\left(\Phi_{e}(s)-\hat{\Phi}_{e}\right)-D_{\Phi_{e}} E_{1}\left(\hat{\Phi}_{\mathrm{tr}}, \hat{\Phi}_{e}, \hat{W}\right)\left(\Phi_{e}(s)-\hat{\Phi}_{e}\right) \\
& \left.\left.+D_{W} E_{1}\left(\hat{\Phi}_{\mathrm{tr}}+\tau \ldots, \hat{\Phi}_{e}+\tau \ldots, \hat{W}+\tau \ldots\right)(W(s)-\hat{W})-D_{W} E_{1}\left(\hat{\Phi}_{\mathrm{tr}}, \hat{\Phi}_{e}, \hat{W}\right)(W(s)-\hat{W})\right) \mathrm{d} \tau\right\rangle \\
& +\left\langle P_{1}, D_{\Phi_{\mathrm{tr}}} E_{1}\left(\hat{\Phi}_{\mathrm{tr}}, \hat{\Phi}_{e}, \hat{W}\right)\left(\Phi_{\mathrm{tr}}(s)-\hat{\Phi}_{\mathrm{tr}}\right)+D_{\Phi_{e}} E_{1}\left(\hat{\Phi}_{\mathrm{tr}}, \hat{\Phi}_{e}, \hat{W}\right)\left(\Phi_{e}(s)-\hat{\Phi}_{e}\right)\right. \\
& \left.\quad+D_{W} E_{1}\left(\hat{\Phi}_{\mathrm{tr}}, \hat{\Phi}_{e}, \hat{W}\right)(W(s)-\hat{W})\right\rangle .
\end{aligned}
$$

By [24], page 133, Corollary 1, we have

$$
\left|\left\langle P_{1}, \int_{0}^{1}(\ldots) \mathrm{d} \tau\right\rangle\right| \leqslant\left\|P_{1}\right\|_{\mathrm{Z}_{1}^{*}} \cdot\left\|\int_{0}^{1}(\ldots) \mathrm{d} \tau\right\|_{\mathrm{Z}_{1}} \leqslant\left\|P_{1}\right\|_{\mathrm{Z}_{1}^{*}} \cdot \int_{0}^{1}\|\ldots\|_{\mathrm{Z}_{1}} \mathrm{~d} \tau .
$$


Consequently, for the first summand within (5.28), it holds that

$$
\begin{aligned}
& \lim _{s \rightarrow 0+0} \frac{1}{s}\left|\left\langle P_{1}, \int_{0}^{1}(\ldots) \mathrm{d} \tau\right\rangle\right| \leqslant \lim _{s \rightarrow 0+0}\left\|P_{1}\right\|_{\mathrm{Z}_{1}^{*}}\left(\int_{0}^{1} \| D_{\Phi_{\mathrm{tr}}} E_{1}\left(\hat{\Phi}_{\mathrm{tr}}+\tau \ldots, \hat{\Phi}_{e}+\tau \ldots, \hat{W}+\tau \ldots\right)\right. \\
& -D_{\Phi_{\mathrm{tr}}} E_{1}\left(\hat{\Phi}_{\mathrm{tr}}, \hat{\Phi}_{e}, \hat{W}\right)\left\|_{\mathcal{L}\left(\widetilde{\mathrm{X}}_{1}, \mathrm{Z}_{1}\right)} \frac{1}{s}\right\| \Phi_{\mathrm{tr}}(s)-\hat{\Phi}_{\mathrm{tr}} \|_{\widetilde{\mathrm{X}}_{1}} \mathrm{~d} \tau \\
& +\int_{0}^{1}\left\|D_{\Phi_{e}} E_{1}\left(\hat{\Phi}_{\mathrm{tr}}+\tau \ldots, \hat{\Phi}_{e}+\tau \ldots, \hat{W}+\tau \ldots,\right)-D_{\Phi_{e}} E_{1}\left(\hat{\Phi}_{\mathrm{tr}}, \hat{\Phi}_{e}, \hat{W}\right)\right\|_{\mathcal{L}\left(\mathrm{X}_{2}, \mathrm{Z}_{1}\right)} \frac{1}{s}\left\|\Phi_{e}(s)-\hat{\Phi}_{e}\right\|_{\mathrm{X}_{2}} \mathrm{~d} \tau \\
& \left.+\int_{0}^{1}\left\|D_{W} E_{1}\left(\hat{\Phi}_{\mathrm{tr}}+\tau \ldots, \hat{\Phi}_{e}+\tau \ldots, \hat{W}+\tau \ldots,\right)-D_{W} E_{1}\left(\hat{\Phi}_{\mathrm{tr}}, \hat{\Phi}_{e}, \hat{W}\right)\right\|_{\mathcal{L}\left(\tilde{\mathrm{X}}_{3}, \mathrm{Z}_{1}\right)} \frac{1}{s}\|W(s)-\hat{W}\|_{\tilde{\mathrm{X}}_{3}} \mathrm{~d} \tau\right) \\
& \leqslant \lim _{s \rightarrow 0+0}\left\|P_{1}\right\|_{\mathrm{Z}_{1}^{*}}\left(\int_{0}^{1} L_{1} \tau\left(\left\|\Phi_{\operatorname{tr}}(s)-\hat{\Phi}_{\mathrm{tr}}\right\|_{\widetilde{\mathrm{X}}_{1}}+\left\|\Phi_{e}(s)-\hat{\Phi}_{e}\right\|_{\mathrm{X}_{2}}+\|W(s)-\hat{W}\|_{\widetilde{\mathrm{X}}_{3}}\right) \frac{1}{s}\left\|\Phi_{\operatorname{tr}}(s)-\hat{\Phi}_{\mathrm{tr}}\right\|_{\widetilde{\mathrm{X}}_{1}} \mathrm{~d} \tau\right. \\
& +\int_{0}^{1} L_{2} \tau\left(\left\|\Phi_{\operatorname{tr}}(s)-\hat{\Phi}_{\mathrm{tr}}\right\|_{\tilde{\mathrm{X}}_{1}}+\left\|\Phi_{e}(s)-\hat{\Phi}_{e}\right\|_{\mathrm{X}_{2}}+\|W(s)-\hat{W}\|_{\tilde{\mathrm{X}}_{3}}\right) \frac{1}{s}\left\|\Phi_{e}(s)-\hat{\Phi}_{e}\right\|_{\mathrm{X}_{2}} \mathrm{~d} \tau \\
& +\int_{0}^{1} L_{3} \tau\left(\left\|\Phi_{\operatorname{tr}}(s)-\hat{\Phi}_{\operatorname{tr}}\right\|_{\tilde{\mathrm{X}}_{1}}+\left\|\Phi_{e}(s)-\hat{\Phi}_{e}\right\|_{\mathrm{X}_{2}}+\|W(s)-\hat{W}\|_{\tilde{\mathrm{X}}_{3}}\right) \frac{1}{s}\|W(s)-\hat{W}\|_{\tilde{\mathrm{X}}_{3}} \mathrm{~d} \tau
\end{aligned}
$$

with Lipschitz constants $L_{1}, L_{2}, L_{3}$, whose existence is ensured by the twice continuous Fréchet differentiability of $E_{1}$ with respect to $\Phi_{\mathrm{tr}}, \Phi_{e}$ and $W$. With reference to Lemma 5.6, the estimate (5.31) may be continued as follows:

$$
\begin{aligned}
& \lim _{s \rightarrow 0+0} \frac{1}{s}\left|\left\langle P_{1}, \int_{0}^{1}(\ldots) \mathrm{d} \tau\right\rangle\right| \\
& \leqslant \lim _{s \rightarrow 0+0}\left\|P_{1}\right\|_{\mathrm{Z}_{1}^{*}} \frac{1}{2}\left(L_{1}+L_{2}+L_{3}\right) \frac{1}{s}\left(\left\|\Phi_{\operatorname{tr}}(s)-\hat{\Phi}_{\mathrm{tr}}\right\|_{\widetilde{\mathrm{X}}_{1}}+\left\|\Phi_{e}(s)-\hat{\Phi}_{e}\right\|_{\mathrm{X}_{2}}+\|W(s)-\hat{W}\|_{\widetilde{\mathrm{X}}_{3}}\right)^{2} \\
& \leqslant \lim _{s \rightarrow 0+0}\left\|P_{1}\right\|_{\mathrm{Z}_{1}^{*}} C\left(\frac{1}{s}\left\|\Phi_{\mathrm{tr}}(s)-\hat{\Phi}_{\mathrm{tr}}\right\|_{\widetilde{\mathrm{X}}_{1}}^{2}+\frac{1}{s}\left\|\Phi_{e}(s)-\hat{\Phi}_{e}\right\|_{\mathrm{X}_{2}}^{2}+\frac{1}{s}\|W(s)-\hat{W}\|_{\tilde{\mathrm{X}}_{3}}^{2}\right)=0
\end{aligned}
$$

and this implies the first of the claimed relations, namely

$$
\begin{array}{r}
\lim _{s \rightarrow 0+0} \frac{1}{s}\left\langle P_{1}, D_{\Phi_{\mathrm{tr}}} E_{1}\left(\hat{\Phi}_{\mathrm{tr}}, \hat{\Phi}_{e}, \hat{W}\right)\left(\Phi_{\mathrm{tr}}(s)-\hat{\Phi}_{\mathrm{tr}}\right)+D_{\Phi_{e}} E_{1}\left(\hat{\Phi}_{\mathrm{tr}}, \hat{\Phi}_{e}, \hat{W}\right)\left(\Phi_{e}(s)-\hat{\Phi}_{e}\right)\right. \\
\left.+D_{W} E_{1}\left(\hat{\Phi}_{\mathrm{tr}}, \hat{\Phi}_{e}, \hat{W}\right)(W(s)-\hat{W})\right\rangle=0 .
\end{array}
$$

From the second and third state equations (3.13) and (3.14), the limit relations (5.24) and (5.25) can be derived in a completely analogous way.

Since $\Phi_{\operatorname{tr}}(s)$ and $W(s)$ take the same initial values as $\hat{\Phi}_{\operatorname{tr}}$ and $\hat{W}$, respectively, it holds further that

$$
\lim _{s \rightarrow 0+0} \frac{1}{s}\left\langle P_{4}, D_{\Phi_{\mathrm{tr}}} E_{4}\left(\hat{\Phi}_{\mathrm{tr}}\right)\left(\Phi_{\mathrm{tr}}(s)-\hat{\Phi}_{\mathrm{tr}}\right)\right\rangle=\lim _{s \rightarrow 0+0} \frac{1}{s}\left\langle P_{5}, D_{W} E_{5}(\hat{W})(W(s)-\hat{W})\right\rangle=0 .
$$

- Step 4. The first variation of the objective. Choose now $\varepsilon>0$ small enough in order to ensure that the difference $F\left(\Phi_{\mathrm{tr}}(s), \Phi_{e}(s), W(s), I_{e}(s)\right)-F\left(\hat{\Phi}_{\mathrm{tr}}, \hat{\Phi}_{e}, \hat{W}, \hat{I}_{e}\right)$ of the objective values is nonnegative for all quadruples $\left(\Phi_{\mathrm{tr}}(s), \Phi_{e}(s), W(s), I_{e}(s)\right)$ belonging to the closed ball $\mathrm{U}_{\varepsilon}\left(\hat{\Phi}_{\mathrm{tr}}, \hat{\Phi}_{e}, \hat{W}, \hat{I}_{e}\right)$ defined in (5.9). As a consequence of 
our assumptions about the integrand $r$, the first variation may be written as

$$
\begin{aligned}
0 \leqslant & \delta^{+} F\left(\hat{\Phi}_{\mathrm{tr}}, \hat{\Phi}_{e}, \hat{W}, \hat{I}_{e}\right)\left(\Phi_{\mathrm{tr}}(1)-\hat{\Phi}_{\mathrm{tr}}, \Phi_{e}(1)-\hat{\Phi}_{e}, W(1)-\hat{W}, I_{e}-\hat{I}_{e}\right) \\
= & \lim _{s \rightarrow 0+0} \frac{1}{s}\left(F\left(\Phi_{\mathrm{tr}}(s), \Phi_{e}(s), W(s), I_{e}(s)\right)-F\left(\hat{\Phi}_{\mathrm{tr}}, \hat{\Phi}_{e}, \hat{W}, \hat{I}_{e}\right)\right) \\
= & \lim _{s \rightarrow 0+0} \frac{1}{s}\left(D_{\Phi_{\mathrm{tr}}} F\left(\hat{\Phi}_{\mathrm{tr}}, \hat{\Phi}_{e}, \hat{W}, \hat{I}_{e}\right)\left(\Phi_{\mathrm{tr}}(s)-\hat{\Phi}_{\mathrm{tr}}\right)+D_{\Phi_{e}} F\left(\hat{\Phi}_{\mathrm{tr}}, \hat{\Phi}_{e}, \hat{W}, \hat{I}_{e}\right)\left(\Phi_{e}(s)-\hat{\Phi}_{e}\right)\right. \\
& \left.\quad+D_{W} F\left(\hat{\Phi}_{\mathrm{tr}}, \hat{\Phi}_{e}, \hat{W}, \hat{I}_{e}\right)(W(s)-\hat{W})+D_{I_{e}} F\left(\hat{\Phi}_{\mathrm{tr}}, \hat{\Phi}_{e}, \hat{W}, \hat{I}_{e}\right)\left(I_{e}(s)-\hat{I}_{e}\right)\right) .
\end{aligned}
$$

Together with Lemma 5.7 and (5.35), we obtain

$$
\begin{aligned}
0 \leqslant & \lim _{s \rightarrow 0+0} \frac{1}{s}\left(D_{\Phi_{\mathrm{tr}}} F\left(\hat{\Phi}_{\mathrm{tr}}, \hat{\Phi}_{e}, \hat{W}, \hat{I}_{e}\right)\left(\Phi_{\mathrm{tr}}(s)-\hat{\Phi}_{\mathrm{tr}}\right)\right. \\
& +\left\langle P_{1}, D_{\Phi_{\mathrm{tr}}} E_{1}\left(\hat{\Phi}_{\mathrm{tr}}, \hat{\Phi}_{e}, \hat{W}\right)\left(\Phi_{\mathrm{tr}}(s)-\hat{\Phi}_{\mathrm{tr}}\right)\right\rangle+\left\langle P_{2}, D_{\Phi_{\mathrm{tr}}} E_{2}\left(\hat{\Phi}_{\mathrm{tr}}, \hat{\Phi}_{e}, \hat{I}_{e}\right)\left(\Phi_{\mathrm{tr}}(s)-\hat{\Phi}_{\mathrm{tr}}\right)\right\rangle \\
& +\left\langle P_{3}, D_{\Phi_{\mathrm{tr}}} E_{3}\left(\hat{\Phi}_{\mathrm{tr}}, \hat{W}\right)\left(\Phi_{\mathrm{tr}}(s)-\hat{\Phi}_{\mathrm{tr}}\right)\right\rangle+\left\langle P_{4}, D_{\Phi_{\mathrm{tr}}} E_{4}\left(\hat{\Phi}_{\mathrm{tr}}\right)\left(\Phi_{\mathrm{tr}}(s)-\hat{\Phi}_{\mathrm{tr}}\right)\right\rangle \\
& +D_{\Phi_{e}} F\left(\hat{\Phi}_{\mathrm{tr}}, \hat{\Phi}_{e}, \hat{W}_{,} \hat{I}_{e}\right)\left(\Phi_{e}(s)-\hat{\Phi}_{e}\right) \\
& +\left\langle P_{1}, D_{\Phi_{e}} E_{1}\left(\hat{\Phi}_{\mathrm{tr}}, \hat{\Phi}_{e}, \hat{W}\right)\left(\Phi_{e}(s)-\hat{\Phi}_{e}\right)\right\rangle+\left\langle P_{2}, D_{\Phi_{e}} E_{2}\left(\hat{\Phi}_{\mathrm{tr}}, \hat{\Phi}_{e}, \hat{I}_{e}\right)\left(\Phi_{e}(s)-\hat{\Phi}_{e}\right)\right\rangle \\
& +D_{W} F\left(\hat{\Phi}_{\mathrm{tr}}, \hat{\Phi}_{e}, \hat{W}, \hat{I}_{e}\right)(W(s)-\hat{W}) \\
& +\left\langle P_{1}, D_{W} E_{1}\left(\hat{\Phi}_{\mathrm{tr}}, \hat{\Phi}_{e}, \hat{W}\right)(W(s)-\hat{W})\right\rangle+\left\langle P_{2}, D_{W} E_{2}\left(\hat{\Phi}_{\mathrm{tr}}, \hat{\Phi}_{e}, \hat{I}_{e}\right)(W(s)-\hat{W})\right\rangle \\
& +\left\langle P_{3}, D_{W} E_{3}\left(\hat{\Phi}_{\mathrm{tr}}, \hat{W}\right)(W(s)-\hat{W})\right\rangle+\left\langle P_{5}, D_{W} E_{5}(\hat{W})(W(s)-\hat{W})\right\rangle \\
& \left.+D_{I_{e}} F\left(\hat{\Phi}_{\mathrm{tr}}, \hat{\Phi}_{e}, \hat{W}, \hat{I}_{e}\right)\left(I_{e}(s)-\hat{I}_{e}\right)+\left\langle P_{2}, D_{I_{e}} E_{2}\left(\hat{\Phi}_{\mathrm{tr}}, \hat{\Phi}_{e}, \hat{I}_{e}\right)\left(I_{e}-\hat{I}_{e}\right)\right\rangle\right)
\end{aligned}
$$

where the first three parts vanish since $P_{1}, P_{2}, P_{3}$ together with $P_{4}=-P_{1}(\cdot, 0)$ and $P_{5}=-P_{3}(\cdot, 0)$ solve the adjoint equations (4.5)-(4.7). Note that, by Section 4.1. above, these equations take the claimed form. Consequently, we arrive at

$$
\begin{aligned}
0 & \leqslant \lim _{s \rightarrow 0+0} \frac{1}{s}\left(D_{I_{e}} F\left(\hat{\Phi}_{\mathrm{tr}}, \hat{\Phi}_{e}, \hat{W}, \hat{I}_{e}\right)\left(I_{e}(s)-\hat{I}_{e}\right)+\left\langle P_{2}, D_{I_{e}} E_{2}\left(\hat{\Phi}_{\mathrm{tr}}, \hat{\Phi}_{e}, \hat{I}_{e}\right)\left(I_{e}-\hat{I}_{e}\right)\right\rangle\right) \\
& =\int_{0}^{T} \int_{\Omega}\left(\mu \hat{I}_{e}-P_{2}\right) \cdot\left(I_{e}-\hat{I}_{e}\right) \mathrm{d} x \mathrm{~d} t=\int_{0}^{T} \int_{\Omega}\left(\mu \hat{I}_{e}-Q P_{2}\right) \cdot\left(I_{e}-\hat{I}_{e}\right) \mathrm{d} x \mathrm{~d} t
\end{aligned}
$$

for arbitrary $I_{e} \in \mathcal{C}$. Since $I_{e}$ and $\hat{I}_{e}$ vanish outside $\Omega_{\mathrm{con}} \times[0, T]$, this confirms the claimed optimality condition (5.3), and the proof is complete.

Proof of Corollary 5.3. Using the representations $I_{e}=Q I$ and $\hat{I}_{e}=Q \hat{I}$, inequality (5.40) may be rewritten as

$$
\begin{aligned}
0 \leqslant & \int_{0}^{T} \int_{\Omega}\left(\mu \cdot Q \hat{I}-Q P_{2}\right) \cdot(Q I-Q \hat{I}) \mathrm{d} x \mathrm{~d} t=\int_{0}^{T} \int_{\Omega}\left(\mu \cdot Q \hat{I}-Q P_{2}\right) \cdot(I-\hat{I}) \mathrm{d} x \mathrm{~d} t \\
= & \int_{0}^{T} \int_{\Omega_{\text {con }}}\left(\mu \cdot Q \hat{I}-Q P_{2}\right) \cdot(I-\hat{I}) \mathrm{d} x \mathrm{~d} t \\
& \forall I \in L^{\infty}\left[(0, T), L^{2}(\Omega)\right] \text { with } \operatorname{supp}(I) \subseteq \Omega_{\text {con }} \times[0, T] \text { and }|I(x, t)| \leqslant R(\forall)(x, t) \in \Omega_{T} .
\end{aligned}
$$

To (5.42), we may apply a Lebesgue point argument analogous to [11], page 1541, Proof of Corollary 3.6., in order to get

$$
\left(\mu \cdot Q \hat{I}\left(x_{0}, t_{0}\right)-Q P_{2}\left(x_{0}, t_{0}\right)\right) \cdot\left(\eta_{0}-\hat{I}\left(x_{0}, t_{0}\right)\right) \geqslant 0 \forall \eta_{0} \in[-R, R](\forall)\left(x_{0}, t_{0}\right) \in \Omega_{\text {con }} \times[0, T],
$$

and this implies the conditions (5.4) and (5.5). 
Proof of Corollary 5.4. This is implied by (5.5) since $Q P_{2} \mid \Omega_{\text {con }} \in L^{2}\left[(0, T), W^{1,2}\left(\Omega_{\text {con }}\right)\right]$ together with $P_{2} \in L^{2}\left[(0, T), W^{1,2}(\Omega)\right]$.

Proof of Corollary 5.5. We can follow the Proof of Theorem 5.2 where only in (5.36), (5.38) and (5.39) the minorization by 0 must be deleted.

Acknowledgements. The present work has been supported within the Special Research Unit 32 "Mathematical Optimization and Applications in Biomedical Sciences "(Graz) by the Austrian Science Fund and the project "Relaxation theorems and necessary optimality conditions for semiconvex multidimensional control problems" (Leipzig) by the German Research Council.

\section{REFERENCES}

[1] B. Ainseba, M. Bendahmane and R. Ruiz-Baier, Analysis of an optimal control problem for the tridomain model in cardiac electrophysiology. J. Math. Anal. Appl. 388 (2012) 231-247.

[2] R.R. Aliev and A.V. Panfilov, A simple two-variable model of cardiac excitation. Chaos, Solitons and Fractals 7 (1996) $293-301$.

[3] M.S. Berger, Nonlinearity and Functional Analysis. Academic Press, New York, San Francisco, London (1977).

[4] Y. Bourgault, Y. Coudière and C. Pierre, Existence and uniqueness of the solution for the bidomain model used in cardiac electrophysiology. Nonlinear Analysis: Real World Appl. 10 (2009) 458-482.

[5] A.J.V. Brandaõ, E. Fernández-Cara, P.M.D. Magalhães and M.A. Rojas-Medar, Theoretical analysis and control results for the FitzHugh-Nagumo equation. Electron. J. Differ. Eq. (2008) 1-20.

[6] B. Dacorogna, Direct Methods in the Calculus of Variations. Springer, New York (2008).

[7] L.C. Evans, Partial Differential Equations. Amer. Math. Soc. Providence (1998).

[8] R. FitzHugh, Impulses and physiological states in theoretical models of nerve membrane. Biophys. J. 1 (1961) $445-466$.

[9] K. Ito and K. Kunisch, Lagrange Multiplier Approach to Variational Problems and Applications. SIAM, Philadelphia (2008).

[10] K. Kunisch, C. Nagaiah and M. Wagner, A parallel Newton-Krylov method for optimal control of the monodomain model in cardiac electrophysiology. Comput. Visualiz. Sci. 14 (2011) [2012], 257-269.

[11] K. Kunisch and M. Wagner, Optimal control of the bidomain system (I): The monodomain approximation with the RogersMcCulloch model. Nonlinear Anal.: Real World Appl. 13 (2012) 1525-1550.

[12] K. Kunisch and M. Wagner, Optimal control of the bidomain system (II): Uniqueness and regularity theorems. University of Graz, Institute for Mathematics and Scientific Computing, SFB-Report No. 2011-008 (to appear: Ann. Mat. Pura Appl.)

[13] S. Muzdeka and E. Barbieri, Control theory inspired considerations for the mathematical model defibrillation, in Proc. of the 44th IEEE Conference on Decision and Control, 2005 and 2005 European Control Conference 7416-7421.

[14] C. Nagaiah and K. Kunisch, Higher order optimization and adaptive numerical solution for optimal control of monodomain equations in cardiac electrophysiology. Appl. Num. Math. 61 (2011) 53-65.

[15] C. Nagaiah, K. Kunisch and G. Plank, Numerical solution for optimal control of the reaction-diffusion equations in cardiac electrophysiology. Comput. Optim. Appl. 49 (2011) 149-178.

[16] C. Nagaiah, K. Kunisch and G. Plank, Optimal control approach to termination of re-entry waves in cardiac electrophysiology. University of Graz, Institute for Mathematics and Scientific Computing, SFB-Report No. 2011-020 (to appear: J. Math. Biol., doi: 10.1007/s00285-012-0557-2)

[17] J. Nagumo, S. Arimoto and S. Yoshizawa, An active pulse transmission line simulating nerve axon. Proc. Institute of Radio Engineers 50 (1962) 2061-2070.

[18] J.M. Rogers and A.D. McCulloch, A collocation-Galerkin finite element model of cardiac action potential propagation. IEEE Trans. Biomed. Engrg. 41 (1994) 743-757.

[19] S. Rolewicz, Funktionalanalysis und Steuerungstheorie. Springer, Berlin, Heidelberg, New York (1976).

[20] J. Sundnes, G.T. Lines, X. Cai, B.F. Nielsen, K.-A.Mardal and A. Tveito, Computing the Electrical Activity in the Heart. Springer, Berlin (2006).

[21] L. Tung, A Bi-Domain Model for Describing Ischemic Myocardial D-C Potentials. Ph.D. thesis. Massachusetts Institute of Technology (1978).

[22] M. Veneroni, Reaction-diffusion systems for the macroscopic bidomain model of the cardiac electric field. Nonlinear Analysis: Real World Appl. 10 (2009) 849-868.

[23] J. Warga, Optimal Control of Differential and Functional Equations. Academic Press, New York, London (1972).

[24] K. Yosida, Functional Analysis. Springer, Berlin (1995) (reprint of the 6th edn. from 1980). 